San Jose State University

SJSU ScholarWorks

Master's Theses

Master's Theses and Graduate Research

Spring 2015

\title{
An Examination of the Mass Limit for Stability at the Triangular Lagrange Points for a Three-Body System and a Special Case of the Four-Body Problem
}

Sean Kemp

San Jose State University

Follow this and additional works at: https://scholarworks.sjsu.edu/etd_theses

\section{Recommended Citation}

Kemp, Sean, "An Examination of the Mass Limit for Stability at the Triangular Lagrange Points for a ThreeBody System and a Special Case of the Four-Body Problem" (2015). Master's Theses. 4546.

DOI: https://doi.org/10.31979/etd.4tf8-hnqx

https://scholarworks.sjsu.edu/etd_theses/4546

This Thesis is brought to you for free and open access by the Master's Theses and Graduate Research at SJSU ScholarWorks. It has been accepted for inclusion in Master's Theses by an authorized administrator of SJSU ScholarWorks. For more information, please contact scholarworks@sjsu.edu. 
AN EXAMINATION OF THE MASS LIMIT FOR STABILITY AT THE TRIANGULAR LAGRANGE POINTS FOR A THREE-BODY SYSTEM AND A SPECIAL CASE OF THE FOUR-BODY PROBLEM

\author{
A Thesis \\ Presented to \\ The Faculty of the Department of Physics and Astronomy \\ San José State University \\ In Partial Fulfillment \\ of the Requirements for the Degree \\ Master of Science
}

by

Sean P. Kemp

May 2015 
(c) 2015

Sean P. Kemp

ALL RIGHTS RESERVED 
The Designated Thesis Committee Approves the Thesis Titled

AN EXAMINATION OF THE MASS LIMIT FOR STABILITY AT THE TRIANGULAR LAGRANGE POINTS FOR A THREE-BODY SYSTEM AND A SPECIAL CASE OF THE FOUR-BODY PROBLEM

by

Sean P. Kemp

APPROVED FOR THE DEPARTMENT OF PHYSICS AND ASTRONOMY

SAN JOSÉ STATE UNIVERSITY

May 2015

Dr. Patrick Hamill

Department of Physics and Astonomy

Dr. Monika Kress

Department of Physics and Astonomy

Dr. Olenka Hubickyj Department of Physics and Astonomy 


\begin{abstract}
AN EXAMINATION OF THE MASS LIMIT FOR STABILITY AT THE TRIANGULAR LAGRANGE POINTS FOR A THREE-BODY SYSTEM AND A SPECIAL CASE OF THE FOUR-BODY PROBLEM
\end{abstract}

\author{
by Sean P. Kemp
}

While the three-body problem has been without an exact analytical solution for some 400 odd years, we have learned a great deal about the dynamics of three-body systems in that time. There are five points relative to two massive bodies where a third body will remain in a stable orbit. These are called the Lagrange points. Two of these points, located at the vertices of equilateral triangles, are stable equilibria. We find clear evidence for these stable points within our own solar system, most notably Jupiter's Trojan satellites.

It may be possible to place a massive body at one of these triangular Lagrange points for the Earth-Moon system. This third massive body might be used to occult bright objects for deep space astronomy or to mine for platinum and other useful materials. The question then arises: how massive an object can one place at one of these triangular Lagrange points? Here we present answers to this question.

There is the possibility of placing an object at both of the triangular Lagrange points simultaneously. We present an analysis of the relative motion of the Earth-Moon system when two massive bodies are added to the configuration. The system will remain stable if any two near Earth asteroids are placed at these Lagrange points. 


\section{ACKNOWLEDGEMENTS}

First and foremost, I would like to thank my thesis adviser, Dr. Patrick

Hamill, for sharing knowledge and expertise. Time and time again he gave sage-like advice that helped me along this path. I would also like to thank the other members of the committee for assisting with this project. I would like to acknowledge the giants (great scientists like Lagrange, Newton, Kepler, etc.) whose shoulders I stand upon to present this research. Thanks also to my family, friends, and colleagues for all the support I have been given throughout this process. 


\section{Table of Contents}

Acknowledgement $\quad$ V

1. Introduction 1

1.1 Frames of Reference 3

1.2 Orbital Motion 4

1.3 The Two-Body Problem 4

$\begin{array}{ll}1.4 \text { The Three-Body Problem } & 7\end{array}$

1.5 The Circular Restricted Three-Body Problem 8

1.6 The Statement of the Problem 9

2. Elements of Celestial Mechanics 10

$\begin{array}{ll}2.1 \text { Orbit Elements } & 10\end{array}$

2.2 Conventions on Physical Quantities 12

2.3 Transformation From an Inertial Frame of Reference to a Rotating

$\begin{array}{ll}\text { Frame } & 13\end{array}$

2.4 The Separation Between the Primary and Secondary Masses 13

3. The Circular Restricted Three-Body Problem and the Existence of Lagrange Points 16

3.1 Working Assumptions 16

$\begin{array}{ll}3.1 .1 \text { Position Vectors } & 16\end{array}$

$\begin{array}{ll}\text { 3.1.2 Newton's Second Law } & 17\end{array}$

$\begin{array}{ll}\text { 3.1.3 The Equations of Motion } & 18\end{array}$

$\begin{array}{ll}\text { 3.1.4 The Total Energy } & 18\end{array}$

3.1.5 Total Angular Momentum 22

3.1.6 Lagrange's Solutions 23

4. The Stability of the Lagrange Points 28

$\begin{array}{ll}4.1 \text { Coordinate Systems } & 28\end{array}$

4.2 Forces and Motion 30

4.3 The Pseudo-Potential 31

4.4 Surfaces of Zero Velocity 35

$\begin{array}{ll}4.5 \text { Analysis } & 37\end{array}$

4.6 The Stability of the Lagrange Points 43

4.7 A Satellite With Nonzero Mass 51

5. The Disturbing Function 54 
6. The Stability of the Lagrange Points 57

6.1 The Net Effect at the 1\% Threshold 61

6.2 Gascheau's Stability Condition 65

7. A Special Case of the Four-Body Problem 68

8. Concluding Remarks 81

References $\quad 83$

Appendix: Summary of Gascheau's Original Work 85 


\section{List of Figures}

1. The Two-Body Problem. Masses $m_{1}$ and $m_{2}$ are located at $\vec{R}_{1}$ and $\vec{R}_{2}$ with respect to the origin $O$. The vector $\vec{r}$ is the position of $m_{2}$ with respect to $m_{1}$.

2. Eccentricity of a massive body orbiting another body. The semi-major axis is $a$, the center of the ellipse is $C$, and the focus of the ellipse is $S$.

3. Inertial to rotating frame coordinate transformation. Here $\xi$ and $\eta$ are the inertial axes, and $x$ and $y$ are the rotating axes.

4. Primary and secondary $x$-positions relative to the center of mass. Values to the right are positive. Thus, the $x$-coordinate of the primary mass is negative.

5. Position vectors of the primary, secondary, and satellite relative to the origin $(O)$ are $\vec{R}_{1}, \vec{R}_{2}$, and $\vec{R}_{0}$. The relative position vectors between the bodies are $\vec{r}, \vec{r}_{1}$, and $\vec{r}_{2}$.

6. The Lagrange points. The collinear Lagrange points are $L_{1}, L_{2}$, and $L_{3}$. The triangular Lagrange points are $L_{4}$ and $L_{5}$.

7. The location of masses with respect to the center of mass for the circular restricted three-body problem. The primary mass is on the lower left, the secondary on the lower right, and the particle is located at the top center. The center of mass is at the origin $\mathrm{O}$.

8. The inertial axes $\xi$ and $\eta$ with the rotating axes $x$ and $y$.

9. Surfaces of zero velocity. Note that the surfaces about the primary (Earth) and secondary (Moon) which intersect are the Hill Spheres about these masses.

10. The distances $x_{1}, x_{2}$, and $x_{0}$ are taken with respect to the center of mass. (The $L_{1}$ case is shown.)

11. The center of mass for a three-body system with a nonzero mass satellite. The upper cross is the location of the new center of mass, and its coordinates are its position relative to the old center of mass. Note that the old center of mass's coordinates are $(0,0)$. 
12. The inertial and corotating frames for the CR3BP with a nonzero mass satellite. $\xi$ and $\eta$ are the inertial axes, $x$ and $y$ are the rotating axes, and the concentric rings are the circular paths that the three masses take as they corotate about the system's center of mass. The relative sizes of the circular paths are not to scale for the Earth-Moon System.

13. The position vectors for the Earth, Moon, and satellite with respect to an inertial origin are $\vec{R}_{1}, \vec{R}_{2}$, and $\vec{R}_{0}$. The position vectors for the Moon and satellite relative to the Earth are $\vec{r}$ and $\vec{r}_{1}$.

14. Positions of the three massive bodies relative to the inertial origin $(O)$. The position of the satellite with respect to the center of mass $(C)$ of the Earth and Moon is denoted by $\vec{\rho}$. See

Figure 3 and discussion in Chapter 3 for remaining notation.

15. Gravitational forces of masses 1 and 2 on the Moon and their orthogonal components. Note that $\vec{F}_{1_{y}}$ and $\vec{F}_{2_{y}}$ are equal in magnitude and opposite in direction, and $\vec{F}_{1_{x}}$ and $\vec{F}_{2_{x}}$ are directed toward the Earth.

16. Relative positions of the $i^{\text {th }}$ and $j^{\text {th }}$ masses with respect to the origin $(O)$ at the primary mass.

17. The effect of $\frac{d\left(\delta n_{i}\right)}{d t}$ on $\theta_{i}-\theta_{j}$. Figure 17 a shows that $\left(\theta_{i}-\theta_{j}\right)$ is greater than $60^{\circ}$, and $\frac{d\left(\delta n_{i}\right)}{d t}$ will move $m_{i}$ back toward the Lagrange point. In Figure $17 \mathrm{~b}$, the angular separation between $m_{i}$ and $m_{j}$ is less than $60^{\circ}$, and $\frac{d\left(\delta n_{i}\right)}{d t}$ increases $\left(\theta_{i}-\theta_{j}\right)$ until $m_{i}$ reaches the Lagrange point. Note that $\delta n_{i}$ and $\frac{d\left(\delta n_{i}\right)}{d t}$ point in opposite directions.

18. The final page of Gascheau's work. 


\section{CHAPTER 1}

\section{INTRODUCTION}

One of Plato's dialogues defines science as knowledge that produces physical results (Plato, c. 380 BCE). To the ancient Greeks, science was the study of the universe about us. Their approach was more philosophical than what we would consider science today. For example, Aristotelian physics includes philosophical considerations (Aristotle, c. 330 BCE). Rene Descartes (1641) separated all substance into "matter" (or "natural") and "mind" (or "unnatural"). We now refer to the study of the natural world predating modern sciences as "natural philosophy." That is not to say that significant advances in the natural sciences were not made in the ancient world; it was perhaps Pythagoras who deduced that the world is round by the shape of its shadow on the Moon during a lunar eclipse (Laërtius, c. 230). Simlarly, Archimedes (c. 250 BCE) is rightfully acknowledged for our understanding of buoyancy and density.

The beginning of modern science can be considered to coincide with the idea that the Earth is not the center of the universe. In the year that he died, Nicolaus Copernicus published a work showing that calculating the motion of celestial bodies such as the planets is both much easier and more precise when assuming that the Sun is at the center of the solar system (Copernicus, 1543). Galileo Galilei would further corroborate this model (as well as perform several key founding experiments in physics) with the discovery of sunspots, the moons of Jupiter, and the phases of other planets (Galilei, 1613). He also designed and built the telescope that allowed him to make these observations.

Around the same time, Johannes Kepler extensively studied the motion of the planets (Kepler, 1621). This led him to the forumlation of his laws of planetary 
motion. As we shall see later, Kepler's third law is of particular interest. That is, the square of a planet's orbital period is proportional to the cube of its semi-major axis.

$$
T^{2}=\frac{4 \pi^{2} a^{3}}{G\left(M_{1}+M_{2}\right)} .
$$

In 1687, Sir Isaac Newton published an iconic text called Philosophice Naturalis Principia Mathematica (or "Mathematical Principles in Natural Philosophy") (Newton, 1687). In this work, Newton formulated his laws of motion; they have since become some of the fundamental principles of physics. In particular, Newton's second law states that the force impressed upon an object is equal to the time derivative of its momentum; for the case of constant mass, this reduces to the familiar "force equals mass times acceleration." That is

$$
\vec{F}=\frac{d \vec{p}}{d t}=m \vec{a}
$$

Newton also formulated his universal law of gravitation (Newton, 1687). That is, two masses will gravitationally attract each other with a force proportional to the product of their masses and inversely proportional to the square of their separation.

$$
\vec{F}=-G \frac{m_{1} m_{2}}{r^{3}} \vec{r}
$$

The Principia Mathematica also addressed the motion of massive bodies in motion under a variety of conditions. In Proposition 66 of the book, Newton defined a situation which would eventually become known as the three-body problem. This problem has been without an exact analytical solution for more than 400 years.

Giuseppe Luigi Comte de Lagrange was both a briliant mathematician and celestial mechanician. Lagrange (1811) was responsible for many of the greatest advances in understanding of the three-body problem. He proved that there are five points in space near two massive bodies where a third body will remain in equilibrium with respect to the first two. They are called Lagrange points. 
Lagrange showed that the two points at the vertices of equilateral triangles (called the "triangular Lagrange points") are stable. Clusters of asteroids have been found to be at these stable points for the Jupiter-Sun system (Nicholson, 1906). These asteroids librate about Jupiter's triangular Lagrange points (Roy, 2005).

Gabriel Gascheau (1843) calculated the upper mass limit at the triangular Lagrange points for a three-body system. If we take the smallest of these masses to be zero, the expression yields a value called the critical mass ratio (Routh, 1875). As long as the primary and secondary masses are in this ratio, the three-body system is stable.

Since then, great advances have been made in our understanding of the three-body problem. Some of these advances will be of particular interest, as we will soon see. A. E. Roy (2005) and also Murray and Dermott (1999) have chronicled much of the body of knowledge of celestial mechanics. Brown and Shook (1933) were able to show how the orbit of a mass orbiting a very massive body will be perturbed by a third mass corotating about the system's center of mass. Salo and Yoder (1988) were able to use this information to show that stable configurations besides the equilateral triangle solutions may exist. In fact, they were able to show in general how the relative motion of one of the smaller bodies orbiting the central mass of the system is affected by the other masses.

\subsection{Frames of Reference}

We shall assume an inertial frame of reference so that Newton's laws can be taken to be valid. We will also consider motion in rotating reference frames. These frames are not inertial, but they can be generated from inertial frames with the appropriate tranformations. While it may be true that relativistic considerations are 
often necessary in astrophysics, we do not require Einstein's (1920) theory since the velocities of the bodies in question are far below relativistic speeds.

\subsection{Orbital Motion}

Orbital motion involves one massive body revolving about another massive body, such as a planet about the Sun or the Moon about the Earth. This could just as easily describe the motion of a satellite about the Earth, the Moon, or Pluto. Of particular interest to us will be the motion of three gravitationally interactive bodies. Let us begin with a simpler case: the two-body problem (Roy, 2005).

\subsection{The Two-Body Problem}

The simplest classical case of gravitational interaction is the attraction between two bodies. The goal of the two-body problem is to find the positions and velocities of these bodies at some later time given the positions, velocities, and masses of two bodies at some initial time. Kepler and Newton studied this problem extensively. In fact, it was the study of this type of motion (namely the motion of Mars about the Sun) that led Kepler to formulate his laws of planetary motion (Kepler, 1621). The consequences of this study have yielded calculations of the masses of planets and moons and the elliptical velocities of orbiting bodies.

The solution of the two-body problem is well known. Two gravitationally interactive bodies are known to corotate about a common center of mass tracing out a conic section. The energy of the orbit of the body governs the type of conic section. Orbits with negative energy are ellipses.

Suppose we have two massive bodies, $m_{1}$ and $m_{2}$. Their position vectors

relative to some fixed reference point $O$ are $\vec{R}_{1}$ and $\vec{R}_{2}$ respectively. See Figure 1. By Newton's second law and Newton's law of universal gravitation, we can describe the movement of the two bodies by the following equations of motion: 


$$
\begin{gathered}
m_{1} \frac{d^{2} \vec{R}_{1}}{d t^{2}}=G \frac{m_{1} m_{2} \vec{r}}{r^{3}}, \\
m_{2} \frac{d^{2} \vec{R}_{2}}{d t^{2}}=-G \frac{m_{1} m_{2} \vec{r}}{r^{3}},
\end{gathered}
$$

where $\vec{r}$ is the position of $m_{2}$ with respect to $m_{1}$, and $G$ is the gravitational constant with a value of $6.638 \times 10^{-11} \frac{\mathrm{m}^{3}}{\mathrm{~kg} \cdot \mathrm{s}^{2}}$. Note that we can scale $G$ to unity by a careful choice of a system of units and thereby omit it in the future.

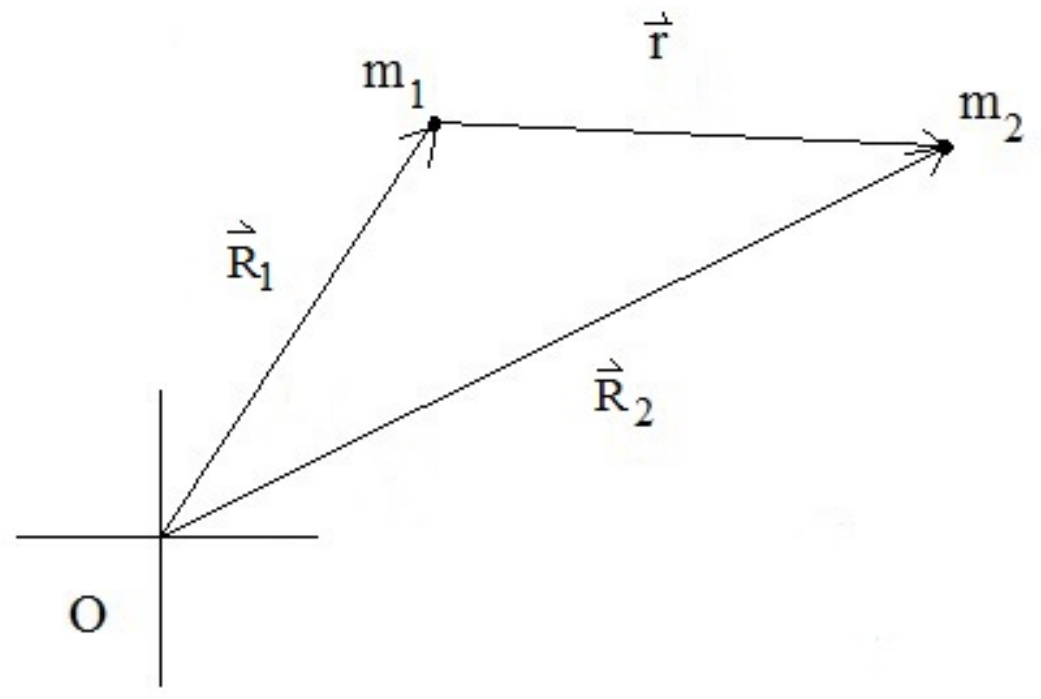

Figure 1. The Two-Body Problem. Masses $m_{1}$ and $m_{2}$ are located at $\vec{R}_{1}$ and $\vec{R}_{2}$ with respect to the origin $O$. The vector $\vec{r}$ is the position of $m_{2}$ with respect to $m_{1}$.

Dividing the Equation 1.1 by $m_{1}$ and Equation 1.2 by $m_{2}$ while scaling $G$ to unity yields 


$$
\begin{gathered}
\frac{d^{2} \vec{R}_{1}}{d t^{2}}=\frac{m_{2} \vec{r}}{r^{3}}, \\
\frac{d^{2} \vec{R}_{2}}{d t^{2}}=-\frac{m_{1} \vec{r}}{r^{3}} .
\end{gathered}
$$

Subtracting Equation 1.4 from Equation 1.3 gives

$$
\frac{d^{2}}{d t^{2}}\left(\vec{R}_{1}-\vec{R}_{2}\right)=\left(m_{1}+m_{2}\right) \frac{\vec{r}}{r^{3}}
$$

Since $\vec{r}=\vec{R}_{2}-\vec{R}_{1}$,

$$
\frac{d^{2} \vec{r}}{d t^{2}}+\left(m_{1}+m_{2}\right) \frac{\vec{r}}{r^{3}}=0 .
$$

Taking the dot product of Equation 1.5 with $\dot{\vec{r}}$,

$$
\dot{\vec{r}} \cdot \frac{d^{2} \vec{r}}{d t^{2}}+\left(m_{1}+m_{2}\right) \frac{\dot{\vec{r}} \cdot \vec{r}}{r^{3}}=0 .
$$

Let us consider the derivative

$$
\begin{aligned}
& \frac{d}{d t}(\dot{\vec{r}} \cdot \dot{\vec{r}})=2(\dot{\vec{r}} \cdot \ddot{\vec{r}})=2\left(\dot{\vec{r}} \cdot \frac{d^{2} r}{d t^{2}}\right), \\
& \frac{1}{2} \frac{d}{d t}(\dot{\vec{r}} \cdot \dot{\vec{r}})=(\dot{\vec{r}} \cdot \ddot{\vec{r}})=\left(\dot{\vec{r}} \cdot \frac{d^{2} r}{d t^{2}}\right) .
\end{aligned}
$$

Since $\vec{r}=r \hat{r}$ and $\dot{\vec{r}}=\dot{r} \hat{r}+r \dot{\theta} \hat{\theta}$,

$$
\dot{\vec{r}} \cdot \vec{r}=r \hat{r} \cdot(\dot{r} \hat{r}+r \dot{\theta} \hat{\theta})=\dot{r} r(\hat{r} \cdot \hat{r})=\dot{r} r .
$$

Thus the second term in Equation 1.6 can be written as

$$
\left(m_{1}+m_{2}\right) \frac{\dot{\vec{r}} \cdot \vec{r}}{r^{3}}=\left(m_{1}+m_{2}\right) \frac{\dot{r} r}{r^{3}}=\frac{\left(m_{1}+m_{2}\right)}{r^{2}} \frac{d r}{d t} .
$$

Substituting Equations 1.7 and 1.8 into 1.6,

$$
\frac{1}{2} \frac{d}{d t}(\dot{\vec{r}} \cdot \dot{\vec{r}})+\frac{\left(m_{1}+m_{2}\right)}{r^{2}} \frac{d r}{d t}=0 .
$$


Multiplying by $d t$ and integrating,

$$
\frac{1}{2} \int d(\dot{\vec{r}} \cdot \dot{\vec{r}})+\left(m_{1}+m_{2}\right) \int \frac{d r}{r^{2}}=0
$$

So

$$
\frac{1}{2} \dot{\vec{r}} \cdot \dot{\vec{r}}-\frac{\left(m_{1}+m_{2}\right)}{r}=\mathrm{constant}=E
$$

Noting that the dot product of $\dot{\vec{r}}$ with itself is the square of the velocity,

$$
\frac{1}{2} v^{2}-\frac{\left(m_{1}+m_{2}\right)}{r}=E,
$$

where $E$ is a constant called the energy constant. Note that $E$ is not the total energy. The first term is related to the kinetic energy, and the second term is related to the potential energy.

\subsection{The Three-Body Problem}

The traditional version of the three-body problem involves three mutually gravitationally attractive bodies (Lagrange, 1811). Given the masses, positions, and velocities of the three bodies at some initial time, the objective of the problem is to calculate the positions and velocities of the bodies at some later time. The three-body problem has been without an exact analytical solution for several hundred years. The three-body problem also arises in quantum mechanics and electromagnetism, and neither of these forms of the problem have been solved.

Nevertheless, significant progress has been made on this perennial problem. We have learned much from the study of this problem in many of its specialized forms. Of particular interest to the study of orbital motion and gravitational interaction is the circular restricted three-body problem (Lagrange, 1811). The study of this version of the three-body problem has led to several important results. 


\subsection{The Circular Restricted Three-Body Problem}

The circular restricted three-body problem involves two massive bodies and one of infinitesimal mass (Lagrange, 1811). The two massive bodies are constrained to move in circles about their mutual center of mass while attracting the infinitesimal mass. The infinitesimal mass does not gravitationally affect the primary and secondary masses. Given the masses, positions, and velocities of the two massive bodies (or equivalently their masses and orbits) the aim of this problem is to compute the position and velocity of the infinitesimal mass (commonly called the "particle" or "satellite") if its position and velocity are known at some given time or "epoch."

In the general three-body problem in ordinary three dimensional space, each body requires three equations to describe its position and three more to describe its velocity. Therefore we must have 18 first order differential equations to describe the motion of the system. Each of these differential equations requires a constant of the motion to solve, but only 10 of the 18 required constants have been discovered. The first six come from the fact that the center of mass of the system moves through space with a constant velocity. The next three are based on the fact that the total angular momentum of the system is constant. The tenth and final constant of the motion is the total energy of the system (Roy, 2005).

The circular restricted three-body problem reduces that number from 18 to 6 , since the motions of the massive bodies are already predetermined. If we restrict the particle to move only in the orbital plane, this further reduces the number of required equations from 6 to 4 (since we are neither considering its position nor its velocity out of the orbital plane). 


\subsection{The Statement of the Problem}

If we approximate two massive bodies as point masses, we can appreciate that there is a point in space between the two massive bodies where a third body will be able to remain in a stable orbit. If these two masses are corotating about a common center of mass, we will see later that there are five of these points. These are called Lagrange points. We will show that three of these Lagrange points are unstable equilibrium points. These all fall on a straight line connecting the two massive bodies. The other two Lagrange points are at the apexes of equilateral triangles with the primary and secondary masses at the base vertices. These two triangular Lagrange points are stable equilibrium points.

Astronomers have long deliberated over the usefulness of placing a massive body at these triangular Lagrange points for the purpose of occulting distant stars for deep space astronomy (Sagan, 1980). Popular fiction has even suggested taking ice from a comet placed at one of these triangular Lagrange points to combat global warming or placing a near Earth asteroid at one of these points to mine it for materials (Stephenson, 2015).

However, the question remains: How massive an object can be placed at these triangular Lagrange points? Is there a limit? Will the configuration remain stable? The balance of this thesis explores these questions. 


\section{CHAPTER 2}

\section{ELEMENTS OF CELESTIAL MECHANICS}

In this chapter we will define the orbit elements and transform from an inertial frame to a rotating frame. The orbit elements are a set of parameters which are used to describe the motion of a celestial body. We transform to a rotating coordinate frame because the problem we are considering is more easily analyzed in this non-inertial reference frame.

\subsection{Orbit Elements}

It is common practice in astronomy to define a body's position and orbital path with six orbit elements plus the time (Roy, 2005). We traditionally take the frame of reference to be a vast sphere with the Sun or the Earth at the center and all heavenly bodies on the surface of the sphere. This sphere is called the celestial sphere. A single fixed point in space is used as a reference. It is denoted the First Point in Aries $(\Upsilon)$.

If we are to consider the orbit of a satellite about the Earth, it is usual to define the orbit elements on a celestial sphere with the Earth at the center. The plane of the apparent path of the Sun across the sky is called the ecliptic. The angular separation between the First Point in Aries and the intersection of the satellite's orbital plane and the ecliptic is called the longitude of the ascending node $(\Omega)$. If the orbital plane and the ecliptic are tilted relative to each other, the angle between them is called the inclination $(i)$. We will only deal with motion in a single

plane, so all angles of inclination will be zero. The point in space where the orbiting body is closest to the Earth is called perigee. The angular separation from the intersection of the plane of the orbit to the projection of perigee on the celestial sphere is called the argument of perigee $(\omega)$. Adding this to the longitude of the 
ascending node gives is the longitude of perigee $(\varpi)$. The sixth orbit element is the time of perigee passage $(\tau)$ or the time or "epoch" when the orbiting body is at perigee. These orbit elements plus the time $(t)$ are sufficient to describe the position and trajectory of a body.

It is well known that the orbit of one body about a more massive body is an ellipse (Kepler, 1621). The semimajor axis $(a)$ of the orbit is half of the longest diameter of the ellipse. The eccentricity $(e)$ is a measure of how elliptical this orbit is compared to a circle. See Figure 2. If $C S$ is the distance from the center of the ellipse to a focus then

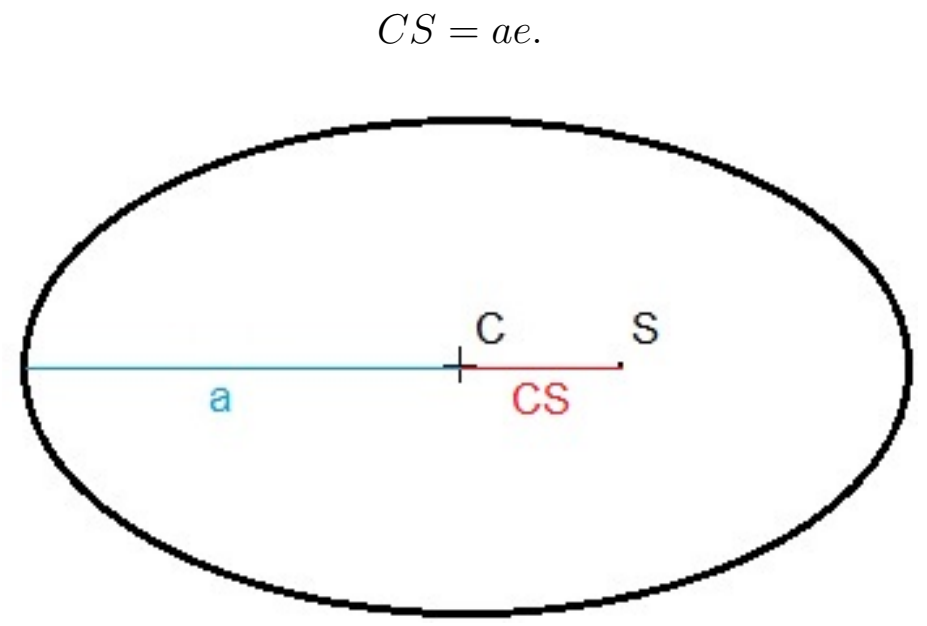

Figure 2. Eccentricity of a massive body orbiting another body. The semi-major axis is $a$, the center of the ellipse is $C$, and the focus of the ellipse is $S$.

For example, the Earth's orbit has an eccentricity of about 0.01671 (Murray and Dermott 1999). Therefore the distance from the center of Earth's orbit $(C)$ to the focus of its orbit ( $S$, the position of the Sun) is about $1.671 \%$ of its semi-major axis. For comparison, the Moon's mean eccentricity is about 5.490\%, and Jupiter's is about $4.839 \%$.

One could question whether it is fair to make the comparison between a system like the Earth-Sun system or the Earth-Moon system and a system in which the 
orbits are perfect circles. We can appreciate that the Earth's eccentricity is about a third of that of Jupiter. Furthermore, the Moon's orbital eccentricity is similar in magnitude to that of Jupiter. If the circular restricted three-body problem yields good reults for the Jupiter-Sun system, it should make a good approximation also for the Earth-Sun and Earth-Moon systems. The Trojan asteroids are evidence that the results of the circular restricted three-body problem are applicable to the Jupiter-Sun system. In conclusion, the orbital eccentricities of the Earth and Moon are of no great consequence.

\subsection{Conventions on Physical Quantities}

For circular orbits, the semi-major axis $(a)$ and the separation between two bodies $(r)$ are equal and often scaled to unity. That is

$$
r=a=1
$$

Let us scale the sum of the mass of the larger massive body (hereafter to be known as the "primary" mass) and the smaller massive body (commonly referred to as the "secondary" mass) to unity. The ratio of the secondary mass to the total mass is often represented by $\mu$. Therefore, the mass of the primary is $1-\mu$ and the mass of the secondary is $\mu$. Thus,

$$
\mu+(1-\mu)=1
$$

We can choose a system of units to scale $G$ as we see fit. As mentioned in Section 1.3, $G$ is often scaled to unity for convenience.

Let us define the mean motion $(n)$ of the system to be the average angular velocity of the primary and secondary masses orbiting their common center of mass. Kepler's third law of planetary motion (Kepler, 1621) states that the square of a planet's orbital period is proportional to the cube of its orbital semi-major axis. 


$$
T^{2}=\frac{4 \pi^{2} a^{3}}{G\left(m_{1}+m_{2}\right)} .
$$

Since $n=\frac{2 \pi}{T}$, Kepler's third law may be written as

$$
n^{2} a^{3}=G\left(m_{1}+m_{2}\right) .
$$

Since $G=1, a=1$, and the sum of the masses also equals one, it follows that:

$$
n^{2} a^{3}=G[\mu+(1-\mu)]
$$

So

$$
n=1 .
$$

\subsection{Transformation From an Inertial Frame of Reference to a Rotating Frame}

Now let us transform from an inertial frame of reference to a rotating frame. The entire system is rotating at some mean velocity $n$ about the center of mass of the system. Let us choose the mutually perpendicular coordinate axes of the rotating frame to be $x$ and $y$. Let us then choose a non-rotating (inertial) reference frame to have coordinate axes $\xi$ and $\eta$. Note that we are only considering motion in a single plane. Let the angular separation between the rotating and stationary frames be $\theta=n t$. See Figure 3 .

\subsection{The Separation Between the Primary and Secondary Masses}

Let us call the $x$-position of the primary mass $x_{1}$ and that of the secondary $x_{2}$. Let us also take the center of mass of the system to be the origin. Since the primary and secondary masses are on opposite sides of the center of mass, $x_{1}$ and $x_{2}$ must have opposite signs. Let $x_{1}$ be negative.

Now since $x_{1}$ is negative, we can subtract it from $x_{2}$ to obtain unity.

$$
x_{2}-x_{1}=1
$$




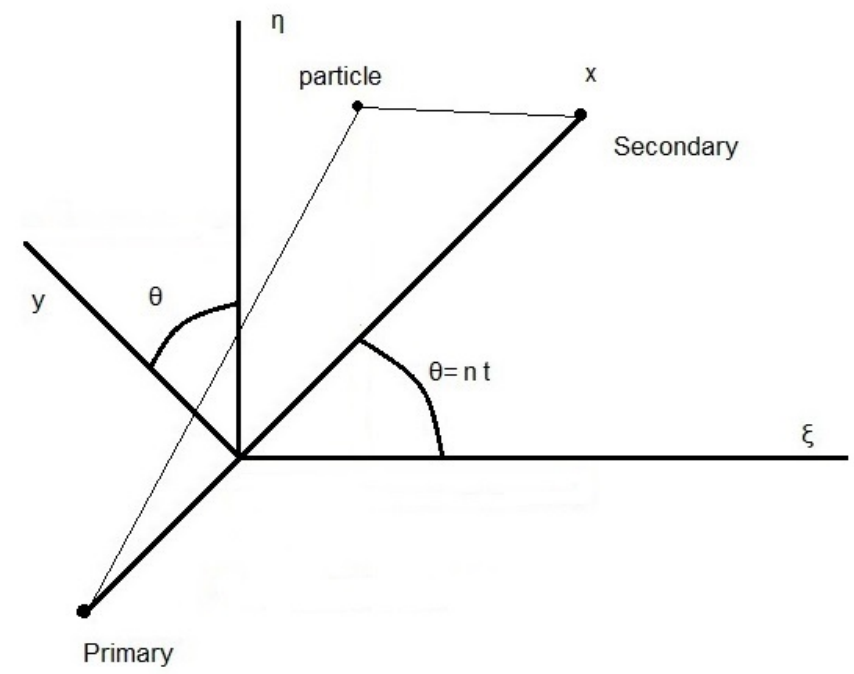

Figure 3. Inertial to rotating frame coordinate transformation. Here $\xi$ and $\eta$ are the inertial axes, and $x$ and $y$ are the rotating axes.

Rearranging for $x_{2}$,

$$
x_{2}=1+x_{1} \text {. }
$$

By the definition of the center of mass,

$$
x_{c m}=\frac{m_{1} x_{1}+m_{2} x_{2}}{m_{1}+m_{2}}=\frac{(1-\mu) x_{1}+\mu x_{2}}{(1-\mu)+\mu} .
$$

Since the center of mass is the origin, and the mass of the primary and secondary sum to unity,

$$
(1-\mu) x_{1}+\mu x_{2}=0
$$

Rearranging for $x_{1}$,

$$
\begin{gathered}
x_{1}=\frac{-\mu x_{2}}{1-\mu}, \\
x_{1}(1-\mu)=-\mu\left(1+x_{1}\right),
\end{gathered}
$$




$$
\begin{gathered}
x_{1}-\mu x_{1}=-\mu-\mu x_{1}, \\
x_{1}=-\mu .
\end{gathered}
$$

Inserting Equation 2.2 into 2.1,

$$
x_{2}=1-\mu \text {. }
$$

The parameters $x_{1}$ and $x_{2}$ are illustrated in Figure 4 .

Now we have developed all the necessary tools to study the three-body

problem in detail. The orbit elements, the separation of the primary and secondary masses from the center of mass, and the transformation from an inertial frame to a rotating frame have laid the groundwork for our analysis. We will use these to determine the existence and positions of the Lagrange points.

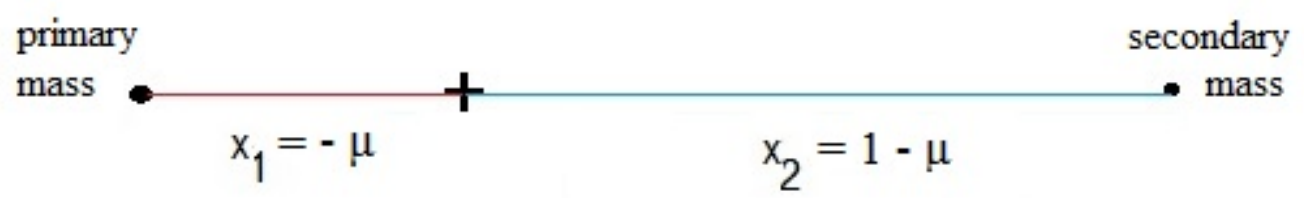

Figure 4. Primary and secondary $x$-positions relative to the center of mass. Values to the right are positive. Thus, the $x$-coordinate of the primary mass is negative. 


\section{CHAPTER 3}

\section{THE CIRCULAR RESTRICTED three-body PROBLEM AND THE EXISTENCE OF LAGRANGE POINTS}

We will show that there are regions of space near two massive corotating bodies where a third body may remain in equilibrium. Let us not be concerned as to whether these equilibrium points are stable or not; we will answer that question in Chapter 4.

\subsection{Lagrange's Analysis}

In 1772, Lagrange showed that the solution to the circular restricted three-body problem (see Section 1.5) led to the following conditions (Roy 2005):

1. The net force on each mass is directed toward the center of mass.

2. This force is proportional to the separation of the mass from the center of mass.

3. The initial velocities of the masses are proportional to the magnitude of their displacements from the center of mass and also make equal angles with the position vectors of the masses relative to the center of mass.

Another way of wording the third condition is to say that the shape of the initial configuration does not change. If the three bodies are initially in an equilateral triangle, the three bodies will always remain in an equilateral triangle even though the size of the triangle is allowed to change.

We will consider these conditions in Section 3.1.6.

\subsubsection{Position Vectors}

Let us call the origin of coordinates $O$. Note that this point does not accelerate in the rotating frame. The mass of the primary is $m_{1}$, the secondary $m_{2}$, and the satellite $m_{0}$. The position vector of the primary mass from the origin $(O)$ shall be 
$\vec{R}_{1}$. Similarly, the respective position vectors of the secondary and satellite relative to the origin are $\vec{R}_{2}$ and $\vec{R}_{0}$. The relative position of the primary mass with respect to the secondary mass is $\vec{r}$. The position of the satellite relative to the primary and secondary are $\vec{r}_{1}$ and $\vec{r}_{2}$. See Figure 5 .

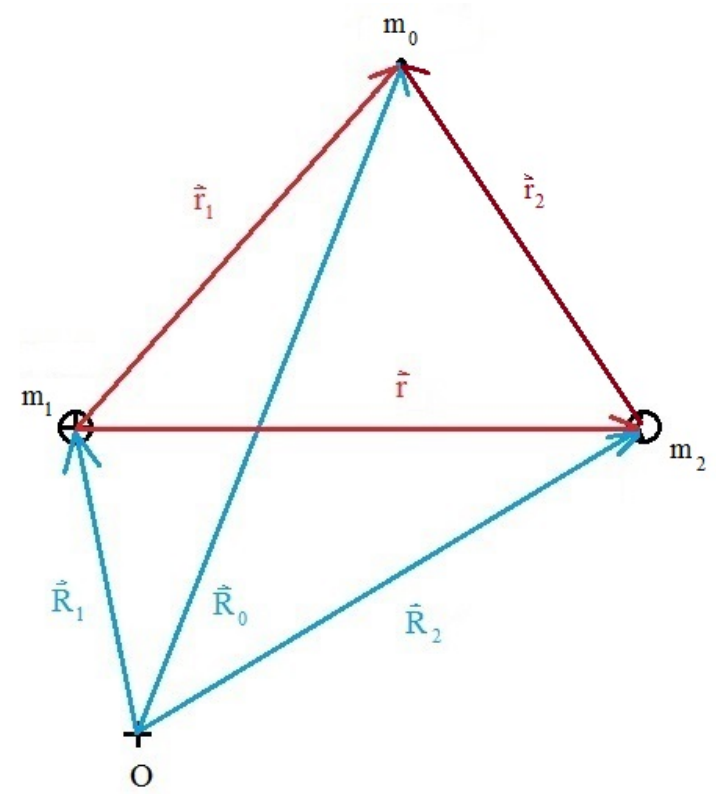

Figure 5. Position vectors of the primary, secondary, and satellite relative to the origin $(O)$ are $\vec{R}_{1}, \vec{R}_{2}$, and $\vec{R}_{0}$. The relative position vectors between the bodies are $\vec{r}, \vec{r}_{1}$, and $\vec{r}_{2}$.

\subsubsection{Newton's Second Law}

Newton's Second Law of Motion states that the net force on an object equals the derivative of its momentum with respect to time.

$$
\vec{F}=\frac{d \vec{p}}{d t} .
$$

Momentum is defined to be the product of an object's mass with its velocity.

$$
\vec{p} \equiv m \vec{v} .
$$


The time derivative of this quantity yields two terms.

$$
\vec{F}=\frac{d \vec{p}}{d t}=m \frac{d \vec{v}}{d t}+\vec{v} \frac{d m}{d t}
$$

If the mass of the object remains constant, the second term is zero. The equation then reduces to:

$$
\vec{F}=m \frac{d \vec{v}}{d t}=m \vec{a}
$$

\subsubsection{The Equations of Motion}

Recall that the general three-body problem has three gravitationally interactive bodies. Let us assume that the only forces acting on these bodies are their mutual gravitation. Therefore, the net force on one of these bodies is the sum of the gravitational forces acting upon it. Instead of using $m_{0}, m_{1}$, and $m_{2}$ let us denote the masses by $m_{i}$ where $i$ goes from 1 to 3 .

$$
m_{i} \ddot{\overrightarrow{R_{i}}}=G \sum_{j=1}^{3} \frac{m_{i} m_{j}}{r_{i j}^{3}} \vec{r}_{i j}(i \neq j),
$$

where $G$ is Newton's gravitational constant. Recall that we have scaled $G$ to unity, yielding

$$
m_{i} \ddot{\overrightarrow{R_{i}}}=\sum_{j=1}^{3} \frac{m_{i} m_{j}}{r_{i j}^{3}} \vec{r}_{i j}(i \neq j) .
$$

\subsubsection{The Total Energy}

Taking the scalar product of the velocities (with respect to the origin) with the equations of motion yields a useful result.

$$
\sum_{i=1}^{3} m_{i} \dot{\vec{R}}_{i} \cdot \ddot{\overrightarrow{R_{i}}}=\sum_{i=1}^{3} \sum_{j=1}^{3} \frac{m_{i} m_{j}}{r_{i j}^{3}} \dot{\vec{R}}_{i} \cdot \vec{r}_{i j}(i \neq j) .
$$

Examining the left hand side of Equation 3.1,

$$
\frac{1}{2} \frac{d}{d t}\left(\dot{\vec{R}}_{i} \cdot \dot{\vec{R}}_{i}\right)=\ddot{\overrightarrow{R_{i}}} \cdot \dot{\vec{R}}_{i}
$$


therefore,

$$
\sum_{i=1}^{3} m_{i} \dot{\overrightarrow{R_{i}}} \cdot \ddot{\overrightarrow{R_{i}}}=\sum_{i=1}^{3} m_{i} \frac{1}{2} \frac{d}{d t}\left(\dot{\vec{R}}_{i} \cdot \dot{\overrightarrow{R_{i}}}\right) .
$$

Multiplying by $d t$ and integrating,

$$
\sum_{i=1}^{3} m_{i} \frac{1}{2} \int d\left(\dot{\vec{R}}_{i} \cdot \dot{\vec{R}}_{i}\right)=\frac{1}{2} \sum_{i=1}^{3} m_{i} \dot{\vec{R}}_{i} \cdot \dot{\vec{R}}_{i}+C .
$$

Let us now examine the right hand side of Equation 3.1. Since this problem concerns three bodies, let us be explicit about each of them.

$$
\begin{gathered}
\sum_{i=1}^{3} \sum_{j=1}^{3} \frac{m_{i} m_{j}}{r_{i j}^{3}} \dot{\vec{R}}_{i} \cdot \vec{r}_{i j}(i \neq j)=\frac{m_{1} m_{2}}{r^{3}} \dot{\vec{R}}_{1} \cdot \vec{r}+\frac{m_{1} m_{0}}{r_{1}^{3}} \dot{\vec{R}}_{1} \cdot \vec{r}_{1} \\
+\frac{m_{2} m_{1}}{r^{3}} \dot{\vec{R}}_{2} \cdot(-\vec{r})+\frac{m_{2} m_{0}}{r_{2}^{3}} \dot{\vec{R}}_{2} \cdot \vec{r}_{2}+\frac{m_{0} m_{1}}{r_{1}^{3}} \dot{\vec{R}}_{0} \cdot\left(-\vec{r}_{1}\right)+\frac{m_{0} m_{2}}{r_{2}^{3}} \dot{\vec{R}}_{0} \cdot\left(-\vec{r}_{2}\right) .
\end{gathered}
$$

Since $\vec{r}_{1}=\vec{R}_{0}-\vec{R}_{1}, \vec{r}_{2}=\vec{R}_{0}-\vec{R}_{2}$, and $\vec{r}=\vec{R}_{2}-\vec{R}_{1}$ the right hand side of Equation 3.1 becomes:

$$
\begin{aligned}
& \frac{m_{1} m_{2}}{r^{3}} \dot{\vec{R}}_{1} \cdot\left(\vec{R}_{2}-\vec{R}_{1}\right)+\frac{m_{1} m_{0}}{r_{1}^{3}} \dot{\vec{R}}_{1} \cdot\left(\vec{R}_{0}-\vec{R}_{1}\right) \\
+ & \frac{m_{2} m_{1}}{r^{3}} \dot{\vec{R}}_{2} \cdot\left(\vec{R}_{1}-\vec{R}_{2}\right)+\frac{m_{2} m_{0}}{r_{2}^{3}} \dot{\vec{R}}_{2} \cdot\left(\vec{R}_{0}-\vec{R}_{2}\right) \\
+ & \frac{m_{0} m_{1}}{r_{1}^{3}} \dot{\vec{R}}_{3} \cdot\left(\vec{R}_{1}-\vec{R}_{0}\right)+\frac{m_{0} m_{2}}{r_{2}^{3}} \dot{\vec{R}}_{3} \cdot\left(\vec{R}_{2}-\vec{R}_{0}\right) .
\end{aligned}
$$

Combining denominators the right hand side of Equation 3.1 becomes:

$$
\begin{aligned}
& \frac{m_{1} m_{2}}{r^{3}}\left[\dot{\vec{R}}_{1} \cdot\left(\vec{R}_{2}-\vec{R}_{1}\right)+\dot{\vec{R}}_{2} \cdot\left(\vec{R}_{1}-\vec{R}_{2}\right)\right] \\
+ & \frac{m_{1} m_{0}}{r_{1}^{3}}\left[\dot{\vec{R}}_{1} \cdot\left(\vec{R}_{0}-\vec{R}_{1}\right)+\dot{\vec{R}}_{0} \cdot\left(\vec{R}_{1}-\vec{R}_{0}\right)\right] \\
+ & \frac{m_{2} m_{0}}{r_{2}^{3}}\left[\dot{\vec{R}}_{2} \cdot\left(\vec{R}_{0}-\vec{R}_{2}\right)+\dot{\vec{R}}_{0} \cdot\left(\vec{R}_{2}-\vec{R}_{0}\right)\right] .
\end{aligned}
$$

Expanding the dot products, the right hand side of Equation 3.1 becomes:

$$
\frac{m_{1} m_{2}}{r^{3}}\left[\dot{\vec{R}}_{1} \cdot \vec{R}_{2}-\dot{\vec{R}}_{1} \cdot \vec{R}_{1}+\dot{\vec{R}}_{2} \cdot \vec{R}_{1}-\dot{\vec{R}}_{2} \cdot \vec{R}_{2}\right]
$$




$$
\begin{aligned}
& +\frac{m_{1} m_{0}}{r_{1}^{3}}\left[\dot{\vec{R}}_{1} \cdot \vec{R}_{0}-\dot{\vec{R}}_{1} \cdot \vec{R}_{1}+\dot{\vec{R}}_{0} \cdot \vec{R}_{1}-\dot{\vec{R}}_{0} \cdot \vec{R}_{0}\right] \\
& +\frac{m_{2} m_{0}}{r_{2}^{3}}\left[\dot{\vec{R}}_{0} \cdot \vec{R}_{0}-\dot{\vec{R}}_{2} \cdot \vec{R}_{2}+\dot{\vec{R}}_{0} \cdot \vec{R}_{0}-\dot{\vec{R}}_{0} \cdot \vec{R}_{0}\right] .
\end{aligned}
$$

Considering the first term in brackets,

$$
\left[\dot{\vec{R}}_{1} \cdot \vec{R}_{2}-\dot{\vec{R}}_{1} \cdot \vec{R}_{1}+\dot{\vec{R}}_{2} \cdot \vec{R}_{1}-\dot{\vec{R}}_{2} \cdot \vec{R}_{2}\right] .
$$

The time derivative of $r^{2}$ is

$$
\begin{gathered}
\frac{d}{d t}\left(r^{2}\right)=\frac{d}{d t}(\vec{r} \cdot \vec{r})=\frac{d}{d t}\left[\left(\vec{R}_{2}-\vec{R}_{1}\right) \cdot\left(\vec{R}_{2}-\vec{R}_{1}\right)\right] \\
=\left[\left(\dot{\vec{R}}_{2}-\dot{\vec{R}}_{1}\right) \cdot\left(\vec{R}_{2}-\vec{R}_{1}\right)+\left(\vec{R}_{2}-\vec{R}_{1}\right) \cdot\left(\dot{\vec{R}}_{2}-\dot{\vec{R}}_{1}\right)\right] \\
=\dot{\vec{R}}_{2} \cdot \vec{R}_{2}-\dot{\vec{R}}_{2} \cdot \vec{R}_{1}-\dot{\vec{R}}_{1} \cdot \vec{R}_{2}+\dot{\vec{R}}_{1} \cdot \vec{R}_{1} \\
+\vec{R}_{2} \cdot \dot{\vec{R}}_{2}-\vec{R}_{2} \cdot \dot{\vec{R}}_{1}-\vec{R}_{1} \cdot \dot{\vec{R}}_{2}+\vec{R}_{1} \cdot \dot{\vec{R}}_{1} \\
=2\left[\dot{\vec{R}}_{1} \cdot \vec{R}_{1}-\dot{\vec{R}}_{1} \cdot \vec{R}_{2}-\dot{\vec{R}}_{2} \cdot \vec{R}_{1}+\dot{\vec{R}}_{2} \cdot \vec{R}_{2}\right],
\end{gathered}
$$

so

$$
-\dot{\vec{R}}_{1} \cdot \vec{R}_{1}+\dot{\vec{R}}_{1} \cdot \vec{R}_{2}+\dot{\vec{R}}_{2} \cdot \vec{R}_{1}-\dot{\vec{R}}_{2} \cdot \vec{R}_{2}=-\frac{1}{2} \frac{d}{d t}\left(r^{2}\right) .
$$

Therefore,

$$
\frac{m_{1} m_{2}}{r^{3}}\left[\dot{\vec{R}}_{1} \cdot \vec{R}_{2}-\dot{\vec{R}}_{1} \cdot \vec{R}_{1}+\dot{\vec{R}}_{2} \cdot \vec{R}_{1}-\dot{\vec{R}}_{2} \cdot \vec{R}_{2}\right]=\frac{m_{1} m_{2}}{r^{3}}\left[-\frac{1}{2} \frac{d}{d t}\left(r^{2}\right)\right] .
$$


It can be shown similarly that,

$$
\begin{aligned}
& \frac{m_{1} m_{0}}{r_{1}^{3}}\left[\dot{\vec{R}}_{1} \cdot \vec{R}_{0}-\dot{\vec{R}}_{1} \cdot \vec{R}_{1}+\dot{\vec{R}}_{0} \cdot \vec{R}_{1}-\dot{\vec{R}}_{3} \cdot \vec{R}_{0}\right]=\frac{m_{1} m_{0}}{r_{1}^{3}}\left[-\frac{1}{2} \frac{d}{d t}\left(r_{1}^{2}\right)\right], \\
& \frac{m_{2} m_{0}}{r_{2}^{3}}\left[\dot{\vec{R}}_{0} \cdot \vec{R}_{0}-\dot{\vec{R}}_{2} \cdot \vec{R}_{2}+\dot{\vec{R}}_{0} \cdot \vec{R}_{0}-\dot{\vec{R}}_{0} \cdot \vec{R}_{0}\right]=\frac{m_{2} m_{3}}{r_{2}^{3}}\left[-\frac{1}{2} \frac{d}{d t}\left(r_{2}^{2}\right)\right] .
\end{aligned}
$$

Therefore the right hand side of Equation 3.1 can be written

$$
\frac{m_{1} m_{2}}{r^{3}}\left[-\frac{1}{2} \frac{d}{d t}\left(r^{2}\right)\right]+\frac{m_{1} m_{0}}{r_{1}^{3}}\left[-\frac{1}{2} \frac{d}{d t}\left(r_{1}^{2}\right)\right]+\frac{m_{2} m_{0}}{r_{2}^{3}}\left[-\frac{1}{2} \frac{d}{d t}\left(r_{2}^{2}\right)\right]
$$

However,

$$
-\frac{1}{2} \frac{d}{d t}\left(r^{2}\right)=-\frac{1}{2} \frac{1}{r^{3}} 2 r \frac{d r}{d t}=-\frac{1}{r^{2}} \frac{d r}{d t}
$$

Thus the right hand side of Equation 3.1 can be written

$$
m_{1} m_{2}\left[-\frac{1}{r^{2}} \frac{d r}{d t}\right]+m_{1} m_{0}\left[-\frac{1}{r_{1}^{2}} \frac{d r_{1}}{d t}\right]+m_{2} m_{0}\left[-\frac{1}{r_{2}^{2}} \frac{d r_{2}}{d t}\right]
$$

Multiplying by $d t$ and integrating,

$$
\begin{gathered}
-m_{1} m_{2} \int \frac{d r}{r^{2}}-m_{1} m_{0} \int \frac{d r_{1}}{r_{1}^{2}}-m_{2} m_{0} \int \frac{d r_{2}}{r_{2}^{2}} \\
=m_{1} m_{2} \frac{1}{r}+m_{1} m_{0} \frac{1}{r_{1}}+m_{2} m_{0} \frac{1}{r_{2}} .
\end{gathered}
$$

Note that since Equation 3.1 sums over both $i$ and $j$, we must introduce a factor of $\frac{1}{2}$ to prevent double summing. Finally generalizing back to $i$ and $j$,

$$
\sum_{i=1}^{3} \sum_{j=1}^{3} \int \frac{m_{i} m_{j}}{r_{i j}^{3}} \dot{\vec{R}}_{i} \cdot \vec{r}_{i j}(i \neq j)=\frac{1}{2} \sum_{i=1}^{3} \sum_{j=1}^{3} \frac{m_{i} m_{j}}{r_{i j}}(i \neq j)+C .
$$


Combining Equations 3.2 and 3.4, we can absorb both of the constants of integration into a single constant $E$, and the integral of Equation 3.1 yields

$$
\frac{1}{2} \sum_{i=1}^{3} m_{i} \dot{\vec{R}}_{i} \cdot \dot{\vec{R}}_{i}-\frac{1}{2} \sum_{i=1}^{3} \sum_{j=1}^{3} \frac{m_{i} m_{j}}{r_{i j}}(i \neq j)=E .
$$

Our constant of integration $(E)$ in Equation 3.5 is the total energy of the system. The first term is the kinetic energy, since

$$
\dot{\vec{R}}_{i} \cdot \dot{\vec{R}}_{i}=V_{i}^{2} .
$$

The second term is the negative of the potential energy.

$$
U=\frac{1}{2} G \sum_{i=1}^{3} \sum_{j=1}^{3} \frac{m_{i} m_{j}}{r_{i j}}(i \neq j) .
$$

Thus Equation 3.5 is the statement of conservation of energy for the system.

\subsubsection{Total Angular Momentum}

If we take the vector product of the position vectors of the masses with their respective equations of motion (see Section 3.1.3), we end up with a useful result.

$$
\sum_{i=1}^{3} m_{i} \vec{R}_{i} \times \ddot{\overrightarrow{R_{i}}}=\sum_{i=1}^{3} \sum_{j=1}^{3} \frac{m_{i} m_{j}}{r_{i j}^{3}} \vec{R}_{i} \times \vec{r}_{i j}(i \neq j) .
$$

Examining the cross product term on the right hand side,

$$
\vec{R}_{i} \times \vec{r}_{i j}=\vec{R}_{i} \times\left(\vec{R}_{j}-\vec{R}_{i}\right)=\vec{R}_{i} \times \vec{R}_{j},
$$

whereas exchanging the subscripts yields

$$
\vec{R}_{j} \times \vec{r}_{j i}=\vec{R}_{j} \times\left(\vec{R}_{i}-\vec{R}_{j}\right)=-\vec{R}_{i} \times \vec{R}_{j}
$$


Summing up these terms for all three bodies, we have terms that cancel in pairs. Therefore the left hand side must be equal to zero.

$$
\sum_{i=1}^{3} m_{i} \vec{R}_{i} \times \ddot{\overrightarrow{R_{i}}}=0 .
$$

Integrating once,

$$
\sum_{i=1}^{3} m_{i} \vec{R}_{i} \times \dot{\overrightarrow{R_{i}}}=C
$$

but $m_{i} \vec{R}_{i} \times \dot{\vec{R}}_{i}$ is the angular momentum of $m_{i}$. Therefore, the total angular momentum of the system must be constant.

\subsubsection{Lagrange's Solutions}

Let us move the origin to the center of mass. Let us define the position vector of $m_{i}$ with respect to the origin to be $\vec{R}_{i}^{\prime}$. Since our origin is at the center of mass, we will begin our analysis with the equation for the center of mass.

$$
\begin{gathered}
\sum_{i=1}^{3} m_{i} \vec{R}_{i}^{\prime}=0, \\
m_{1} \vec{R}_{1}^{\prime}+m_{2} \vec{R}_{2}^{\prime}+m_{0} \vec{R}_{0}^{\prime}=0 .
\end{gathered}
$$

This can be expressed equivalently by

$$
\left(m_{1}+m_{2}+m_{0}\right) \vec{R}_{1}^{\prime}+m_{2}\left(\vec{R}_{2}^{\prime}-\vec{R}_{1}^{\prime}\right)+m_{0}\left(\vec{R}_{0}^{\prime}-\vec{R}_{1}^{\prime}\right)=0,
$$

and since $\vec{r}=\vec{R}_{2}^{\prime}-\vec{R}_{1}^{\prime}$ and $\vec{r}_{1}=\vec{R}_{0}^{\prime}-\vec{R}_{1}^{\prime}$ then,

$$
M \vec{R}_{1}^{\prime}=-m_{2} \vec{r}-m_{0} \vec{r}_{1}
$$

where

$$
M=\left(m_{1}+m_{2}+m_{0}\right)
$$


Squaring,

$$
M^{2}\left(\vec{R}_{1}^{\prime}\right)^{2}=m_{2}^{2} \vec{r}^{2}+m_{3}^{2}{\overrightarrow{r_{1}}}^{2}+2 m_{2} m_{0} \vec{r} \cdot \overrightarrow{r_{1}}
$$

Now if the shape of the system does not change with respect to time, the relative distances between the masses must always keep the same ratios. For initial values $(r)_{0},\left(r_{1}\right)_{0}$, and $\left(r_{2}\right)_{0}$

$$
\frac{r}{(r)_{0}}=\frac{r_{1}}{\left(r_{1}\right)_{0}}=\frac{r_{2}}{\left(r_{2}\right)_{0}}=f(t) .
$$

The angular separations of the masses must be constant. Thus, their angular velocities $(\dot{\theta})$ must be equal (though they may change with time).

$$
\dot{\theta}_{1}=\dot{\theta}_{2}=\dot{\theta}_{3}=\dot{\theta}(t) \text {. }
$$

Rewriting Equation 3.7,

$$
M^{2}\left(\vec{R}_{1}^{\prime}\right)^{2}=[f(t)]^{2}\left[m_{2}^{2}(r)_{0}^{2}+m_{0}^{2}\left(r_{1}\right)_{0}^{2}+2 m_{2} m_{0}(r)_{0}^{2}\left(r_{1}\right)_{0}^{2} \cos \alpha_{1}\right] .
$$

The (constant) angle between $\vec{r}$ and $\vec{r}_{1}$ is $\alpha_{1}=\left(\theta_{2}-\theta_{1}\right)$. Thus,

$$
R_{1}^{\prime}=\left(R_{1}^{\prime}\right)_{0} f(t)
$$

Recall that the total angular momentum of the system is constant. (See Equation 3.6.) Thus, $\dot{\vec{R}}_{i}$ can be written in the following form:

$$
\dot{\vec{R}}_{i}^{\prime}=\left(\dot{R}_{i 0}^{\prime} f\right) \hat{R}_{i 0}^{\prime}+\left(R_{i 0}^{\prime} f\right) \dot{\theta} \hat{\theta}
$$

Taking the cross product with $R_{i 0}^{\prime} f \hat{R}_{i 0}^{\prime}$ yields

$$
R_{i 0}^{\prime} f \hat{R}_{i 0}^{\prime} \times\left[\left(\dot{R}_{i 0}^{\prime} f\right) \hat{R}_{i 0}^{\prime}+\left(R_{i 0}^{\prime} f\right) \dot{\theta} \hat{\theta}\right]=\left(R_{i 0}^{\prime}\right)^{2} f^{2} \dot{\theta} \hat{z}
$$


This is the angular momentum of the $i^{\text {th }}$ body. Therefore the total angular momentum is

$$
\sum_{i=1}^{3} m_{i}\left(R_{i}^{\prime}\right)_{0}^{2} f^{2} \dot{\theta}
$$

From Equation 3.6, the total angular momentum is constant.

$$
\sum_{i=1}^{3} m_{i}\left(R_{i}^{\prime}\right)_{0}^{2} f^{2} \dot{\theta}=\text { constant } .
$$

Therefore, the net torque on each mass must be zero. Since torque is $\vec{\tau}=\vec{r} \times \vec{F}, \vec{F}$ must be parallel to $\vec{r}$. Thus the net force on each mass must pass through the center of mass.

Since the net force is directed radially, it has no tangential component. Now if $\vec{F}_{i}$ is the force per unit mass acting on the $i^{t h}$ mass, the equation of motion for this mass becomes

$$
m_{i} F_{i}=m_{i}\left(\ddot{R}_{i}^{\prime}-R_{i}^{\prime} \dot{\theta}_{i}^{2}\right)
$$

By the second time derivative of Equation 3.8,

$$
m_{i} F_{i}=m_{i}\left[\left(R_{i}^{\prime}\right)_{0} \ddot{f}-R_{i}^{\prime} \dot{\theta}^{2}\right] .
$$

By Equation 3.8,

$$
m_{i} F_{i}=R_{i}^{\prime} m_{i}\left[\frac{\ddot{f}}{f}-\dot{\theta}^{2}\right] .
$$

Note that $F_{i}$ is proportional to $R_{i}^{\prime}$. Therefore,

$$
F_{1}: F_{2}: F_{0}=R_{1}^{\prime}: R_{2}^{\prime}: R_{0}^{\prime}
$$

Two possible conditions that can satisfy this relation are the following:

$$
\begin{aligned}
& \vec{R}_{i}^{\prime} \times \vec{F}_{i}=0, \\
& \vec{R}_{i}^{\prime} \times \ddot{\vec{R}}_{i}^{\prime}=0 .
\end{aligned}
$$


Now let us reexamine our equations of motion.

$$
m_{i} \ddot{\vec{R}}_{i}^{\prime}=\sum_{j=1}^{3} \frac{m_{i} m_{j}}{r_{i j}^{3}} \overrightarrow{r_{i j}}(i \neq j) .
$$

For $i=1$,

$$
m_{1} \ddot{\overrightarrow{R_{1}^{\prime}}}=\sum_{j=2}^{3} \frac{m_{1} m_{j}}{r_{1 j}^{3}} \vec{r}_{1 j}
$$

Dividing by $m_{1}$,

$$
\ddot{\vec{R}}_{1}^{\prime}=\sum_{j=2}^{3} \frac{m_{j}}{r_{1 j}^{3}} \vec{r}_{1 j}=\left(m_{2} \frac{\vec{R}_{2}^{\prime}}{r^{3}}+m_{0} \frac{\vec{R}_{0}^{\prime}}{r_{1}^{3}}\right) .
$$

Taking the vector product of $\vec{R}_{1}^{\prime}$ with Equation 3.9,

$$
\vec{R}_{1}^{\prime} \times \ddot{\vec{R}}_{1}^{\prime}=\vec{R}_{1}^{\prime} \times\left(m_{2} \frac{\vec{R}_{2}^{\prime}}{r^{3}}+m_{0} \frac{\vec{R}_{0}^{\prime}}{r_{1}^{3}}\right) .
$$

Since the force on $m_{1}$ is directed toward the center of mass, the acceleration $\ddot{\vec{R}}_{1}^{\prime}$ is parallel to $\vec{R}_{1}^{\prime}$. Therefore the left hand side of equation 3.10 is zero. Thus,

$$
\vec{R}_{1}^{\prime} \times\left(m_{2} \frac{\vec{R}_{2}^{\prime}}{r^{3}}+m_{0} \frac{\vec{R}_{0}^{\prime}}{r_{1}^{3}}\right)=0 .
$$

Since the origin is at the center of mass,

$$
m_{2} \vec{R}_{1}^{\prime} \times \vec{R}_{2}^{\prime}\left(\frac{1}{r^{3}}-\frac{1}{r_{1}^{3}}\right)=0 .
$$

Now for this expression to equal zero, either the vector product of $\vec{R}_{1}^{\prime}$ and $\vec{R}_{2}^{\prime}$ must be zero or $\vec{r}$ and $\vec{r}_{1}$ must be equal in magnitude. For the vector product of $\vec{R}_{1}^{\prime}$ and $\vec{R}_{2}^{\prime}$ to be zero, the vectors must be parallel. In this case the equilibrium points are collinear with the primary and secondary masses. The other possibility is that $\vec{r}$ and $\vec{r}_{1}$ are equal in magnitude. In this case equilibrium points exist at the apexes of equilateral triangles with the primary and secondary masses at the base vertices. These are the Lagrange points. They are illustrated in Figure 6. 


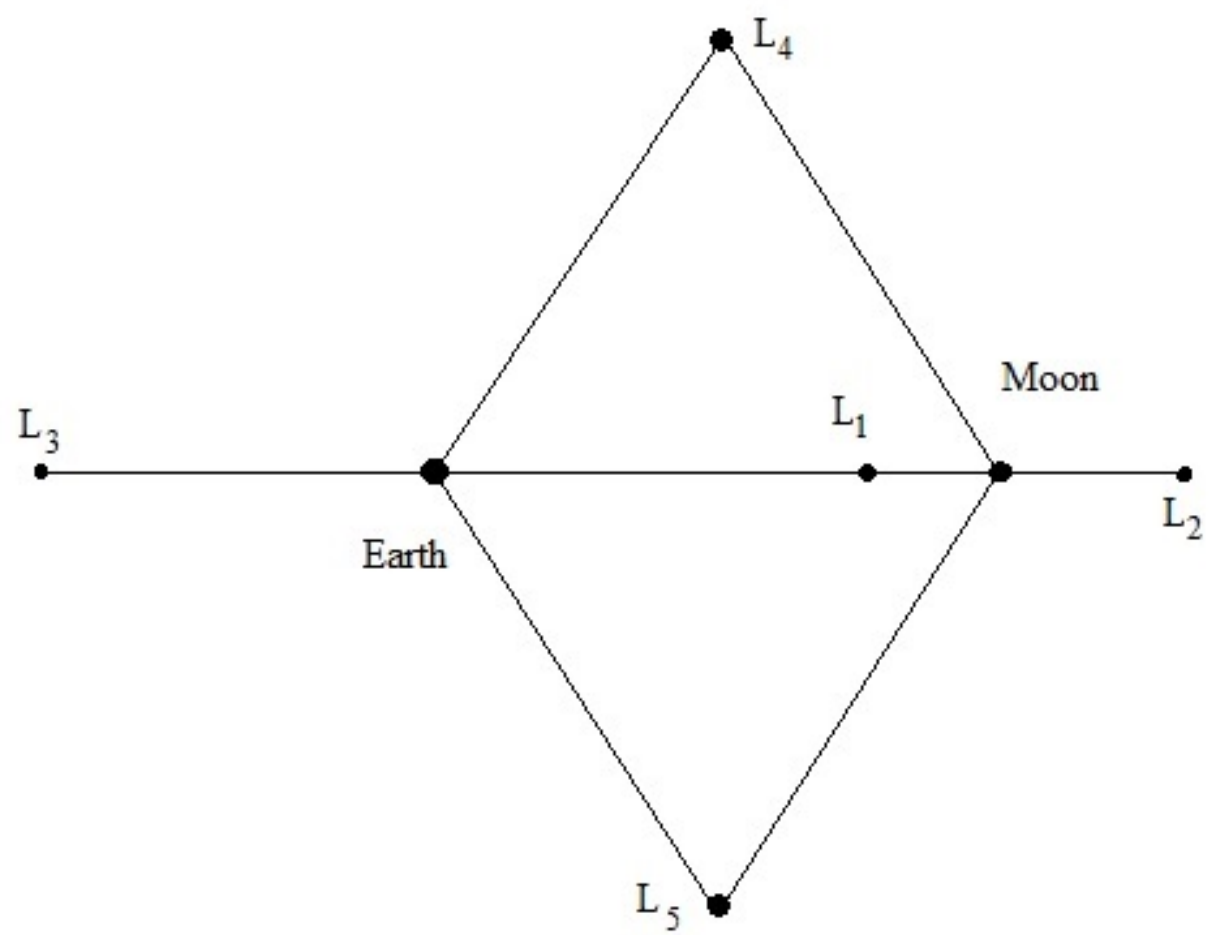

Figure 6. The Lagrange points. The collinear Lagrange points are $L_{1}, L_{2}$, and $L_{3}$. The triangular Lagrange points are $L_{4}$ and $L_{5}$. 


\section{CHAPTER 4}

\section{THE STABILITY OF THE LAGRANGE POINTS}

After having considered the general three-body problem in Chapter 3, we return to the circular restricted three-body problem and consider the stability of the libration (or Lagrange) points. This is done by displacing the infinitesimal mass from one of the Lagrange points by a small amount, by giving it a small velocity, or both. If the body rapidly falls away from the libration point then that Lagrange point is an unstable equilibrium point. However, if the resultant motion oscillates about the Lagrange point, it is a stable equilibrium.

The center of mass in the classic circular restricted three-body problem (CR3BP) is on the line between the primary and secondary masses, a distance $\mu$ from the primary and $(1-\mu)$ from the secondary where the distance between the masses sums to unity (see Section 2.4). The masses of the primary and secondary also sum to unity, where the mass of the primary is $(1-\mu)$ and the mass of the secondary $\mu$. Note that this means the $x$-position of the third body with respect to the center of mass is $\left(\frac{1}{2}-\mu\right)$ and its y-position is $(\sqrt{3} / 2)$ since it is at the vertex of an equilateral triangle whose sides are of length unity.

\subsection{Coordinate Systems}

We shall begin by assuming that the three bodies revolve in circular paths about their common center of mass. Let us also continue to restrict motion to a single plane. The inertial axes continue to be $\xi$ and $\eta$, and the rotating axes remain $x$ and $y$. Let us choose a system of units such that the mean motion is unity, that is $n=1$. Therefore, the angular position of the corotating frame is dependent upon time. Let $\theta=t$. We specify $\left(x_{1}, y_{1}\right)$ to be the location of the primary in the rotating frame, $\left(x_{2}, y_{2}\right)$ to be the location of the secondary, and $\left(x_{0}, y_{0}\right)$ to be the location of 
the particle or satellite. Similarly $\left(\xi_{1}, \eta_{1}\right)$ is the location of the primary in the inertial frame, $\left(\xi_{2}, \eta_{2}\right)$ is the secondary, and $\left(\xi_{0}, \eta_{0}\right)$ is the satellite. As before, $\vec{r}_{1}$ is the position of the satellite relative to the primary mass and $\vec{r}_{2}$ is the position of the satellite relative to the secondary. (See Figure 7.)

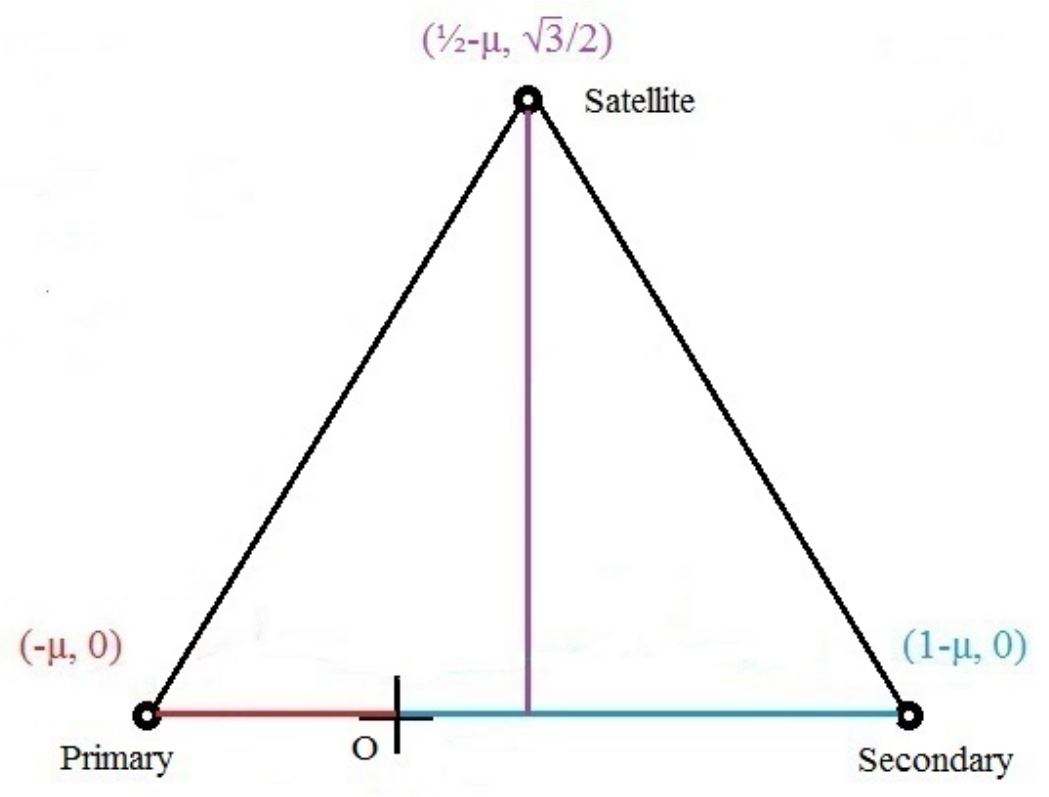

Figure 7. The location of masses with respect to the center of mass for the circular restricted three-body problem. The primary mass is on the lower left, the secondary on the lower right, and the particle is located at the top center. The center of mass is at the origin $\mathrm{O}$.

That is,

$$
\begin{aligned}
& r_{1}^{2}=\left(x_{1}-x_{0}\right)^{2}+\left(y_{1}-y_{0}\right)^{2}=\left(\xi_{1}-\xi_{0}\right)^{2}+\left(\eta_{1}-\eta_{0}\right)^{2}, \\
& r_{2}^{2}=\left(x_{2}-x_{0}\right)^{2}+\left(y_{2}-y_{0}\right)^{2}=\left(\xi_{2}-\xi_{0}\right)^{2}+\left(\eta_{2}-\eta_{0}\right)^{2} .
\end{aligned}
$$

Note that since $n=1$,

$$
\begin{aligned}
& \xi=x \cos t-y \sin t, \\
& \eta=x \sin t+y \cos t .
\end{aligned}
$$


See Figure 8.

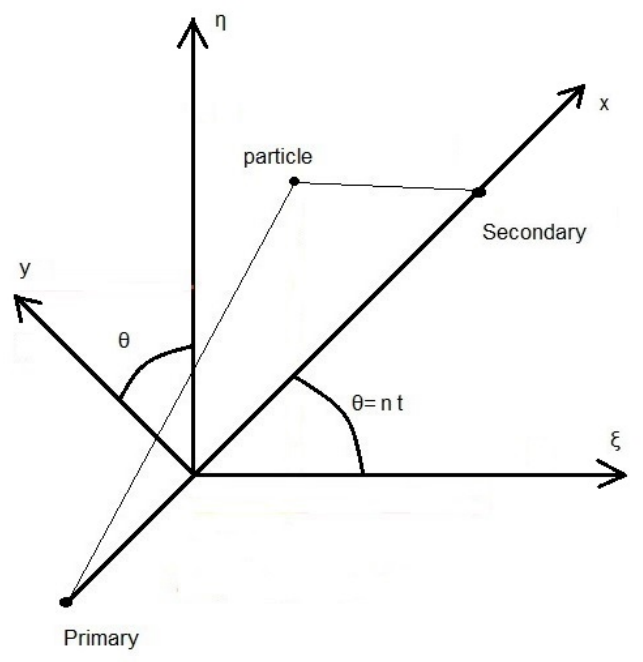

Figure 8. The inertial axes $\xi$ and $\eta$ with the rotating axes $x$ and $y$.

\subsection{Forces and Motion}

The attractive forces between the bodies in this system are gravitational in nature. Therefore by the law of universal gravitation the net force on the satellite is given by

$$
\overrightarrow{F_{0}}=\frac{-m_{0} m_{1} \overrightarrow{r_{1}}}{r_{1}^{3}}+\frac{-m_{0} m_{2} \overrightarrow{r_{2}}}{r_{2}^{3}}
$$

The force per unit mass is

$$
\frac{\overrightarrow{F_{0}}}{m_{0}}=\frac{-m_{1} \overrightarrow{r_{1}}}{r_{1}^{3}}+\frac{-m_{2} \overrightarrow{r_{2}}}{r_{2}^{3}} .
$$

Then the equations of motion of the satellite in the inertial frame are

$$
\begin{aligned}
& \ddot{\xi}_{0}=(1-\mu) \frac{\xi_{1}-\xi_{0}}{r_{1}^{3}}+\mu \frac{\xi_{2}-\xi_{0}}{r_{2}^{3}}, \\
& \ddot{\eta}_{0}=(1-\mu) \frac{\eta_{1}-\eta_{0}}{r_{1}^{3}}+\mu \frac{\eta_{2}-\eta_{0}}{r_{2}^{3}}
\end{aligned}
$$

where $(1-\mu)$ is the mass of the primary and $\mu$ is the mass of the secondary (see Section 2.2). 


\subsection{The Pseudo-Potential}

Let us begin by differentiating the coordinate transformation equations from Section 4.1 (Equations 4.1 and 4.2) twice.

$$
\begin{gathered}
\xi=x \cos t-y \sin t, \\
\dot{\xi}=\dot{x} \cos t-x \sin t-\dot{y} \sin t-y \cos t, \\
\ddot{\xi}=\ddot{x} \cos t-\dot{x} \sin t-\dot{x} \sin t-x \cos t-\ddot{y} \sin t-\dot{y} \cos t-\dot{y} \cos t+y \sin t, \\
\ddot{\xi}=(\ddot{x}-2 \dot{y}-x) \cos t-(\ddot{y}+2 \dot{x}-y) \sin t, \\
\eta=x \sin t+y \cos t, \\
\dot{\eta}=\dot{x} \sin t+x \cos t+\dot{y} \cos t-y \sin t, \\
\ddot{\eta}=\ddot{x} \sin t+\dot{x} \cos t+\dot{x} \cos t-x \sin t+\ddot{y} \cos t-\dot{y} \sin t-\dot{y} \sin t-y \cos t, \\
\ddot{\eta}=(\ddot{y}+2 \dot{x}-y) \cos t+(\ddot{x}-2 \dot{y}-x) \sin t .
\end{gathered}
$$

Setting Equations 4.3 and 4.4 equal to Equations 4.5 and 4.6 respectively,

$$
\begin{aligned}
& \left(\ddot{x}_{0}-2 \dot{y}_{0}-x_{0}\right) \cos t-\left(\ddot{y}_{0}+2 \dot{x}_{0}-y_{0}\right) \sin t=(1-\mu) \frac{\xi_{1}-\xi_{0}}{r_{1}^{3}}+\mu \frac{\xi_{2}-\xi_{0}}{r_{2}^{3}}, \\
& \left(\ddot{y}_{0}+2 \dot{x}_{0}-y_{0}\right) \cos t+\left(\ddot{x}_{0}-2 \dot{y}_{0}-x_{0}\right) \sin t=(1-\mu) \frac{\eta_{1}-\eta_{0}}{r_{1}^{3}}+\mu \frac{\eta_{2}-\eta_{0}}{r_{2}^{3}} .
\end{aligned}
$$

For ease of computation, some quantities will be defined. Let

$$
\begin{aligned}
& a^{\prime}=\left(\ddot{x}_{0}-2 \dot{y}_{0}-x_{0}\right), \\
& b^{\prime}=\left(\ddot{y}_{0}+2 \dot{x}_{0}-y_{0}\right),
\end{aligned}
$$




$$
\begin{aligned}
& c^{\prime}=\left[\frac{(1-\mu)\left(x_{1}-x_{0}\right)}{r_{1}^{3}}+\frac{\mu\left(x_{2}-x_{0}\right)}{r_{2}^{3}}\right], \\
& d^{\prime}=\left[\frac{(1-\mu)\left(y_{1}-y_{0}\right)}{r_{1}^{3}}+\frac{\mu\left(y_{2}-y_{0}\right)}{r_{2}^{3}}\right] .
\end{aligned}
$$

Substituting $a^{\prime}$ and $b^{\prime}$ into Equations 4.7 and 4.8,

$$
\begin{gathered}
a^{\prime} \cos t-b^{\prime} \sin t=\left[\frac{(1-\mu)\left(x_{1}-x_{0}\right)}{r_{1}^{3}}+\frac{\mu\left(x_{2}-x_{0}\right)}{r_{2}^{3}}\right] \cos t \\
+\left[\frac{(1-\mu)\left(y_{1}-y_{0}\right)}{r_{1}^{3}}+\frac{\mu\left(y_{2}-y_{0}\right)}{r_{2}^{3}}\right] \sin t, \\
b^{\prime} \cos t+a^{\prime} \sin t=\left[\frac{(1-\mu)\left(x_{1}-x_{0}\right)}{r_{1}^{3}}+\frac{\mu\left(x_{2}-x_{0}\right)}{r_{2}^{3}}\right] \sin t \\
+\left[\frac{(1-\mu)\left(y_{1}-y_{0}\right)}{r_{1}^{3}}+\frac{\mu\left(y_{2}-y_{0}\right)}{r_{2}^{3}}\right] \cos t .
\end{gathered}
$$

Now also inserting $c^{\prime}$ and $d^{\prime}$ into Equations 4.7 and 4.8,

$$
\begin{aligned}
& a^{\prime} \cos t-b^{\prime} \sin t=c^{\prime} \cos t-d^{\prime} \sin t, \\
& b^{\prime} \cos t+a^{\prime} \sin t=c^{\prime} \sin t+d^{\prime} \cos t .
\end{aligned}
$$

Multiplying Equation 4.9 by $\cos (t)$ and Equation 4.10 by $\sin (t)$,

$$
\begin{aligned}
& a^{\prime} \cos ^{2} t-b^{\prime} \sin t \cos t=c^{\prime} \cos ^{2} t-d^{\prime} \sin t \cos t \\
& b^{\prime} \sin t \cos t+a^{\prime} \sin ^{2} t=c^{\prime} \sin ^{2} t+d^{\prime} \sin t \cos t
\end{aligned}
$$

Adding these two equations,

$$
\begin{gathered}
a^{\prime}\left(\sin ^{2} t+\cos ^{2} t\right)+b^{\prime} \sin t \cos t-b^{\prime} \sin t \cos t=c^{\prime}\left(\sin ^{2} t+\cos ^{2} t\right) \\
+d^{\prime} \sin t \cos t-d^{\prime} \sin t \cos t, \\
a^{\prime}\left(\sin ^{2} t+\cos ^{2} t\right)=c^{\prime}\left(\sin ^{2} t+\cos ^{2} t\right) .
\end{gathered}
$$


So

$$
\begin{gathered}
a^{\prime}=c^{\prime}, \\
\left(\ddot{x_{0}}-2 \dot{y_{0}}-x_{0}\right)=\left[\frac{(1-\mu)\left(x_{1}-x_{0}\right)}{r_{1}^{3}}+\frac{\mu\left(x_{2}-x_{0}\right)}{r_{2}^{3}}\right] .
\end{gathered}
$$

Multiplying Equation 4.9 by $-\cos (\mathrm{t})$ and 4.10 by $\cos (\mathrm{t})$,

$$
\begin{gathered}
-a^{\prime} \sin t \cos t+b^{\prime} \sin ^{2} t=-c^{\prime} \sin t \cos t+d^{\prime} \sin ^{2} t, \\
b^{\prime} \cos ^{2} t+a^{\prime} \sin t \cos t=c^{\prime} \sin t \cos t+d^{\prime} \cos ^{2} t
\end{gathered}
$$

Adding,

$$
\begin{gathered}
b^{\prime}\left(\sin ^{2} t+\cos ^{2} t\right)+a^{\prime} \sin t \cos t-a^{\prime} \sin t \cos t=d^{\prime}\left(\sin ^{2} t+\cos ^{2} t\right) \\
+c^{\prime} \sin t \cos t-c^{\prime} \sin t \cos t, \\
b^{\prime}\left(\sin ^{2} t+\cos ^{2} t\right)=d^{\prime}\left(\sin ^{2} t+\cos ^{2} t\right) .
\end{gathered}
$$

So,

$$
\begin{gathered}
b^{\prime}=d^{\prime}, \\
\left(\ddot{y_{0}}+2 \dot{x_{0}}-y_{0}\right)=\left[\frac{(1-\mu)\left(y_{1}-y_{0}\right)}{r_{1}^{3}}+\frac{\mu\left(y_{2}-y_{0}\right)}{r_{2}^{3}}\right] .
\end{gathered}
$$

Now we have two equations of motion for the satellite. We can simplify these equations of motion by defining a function $U$ called the "Pseudo-Potential" by

$$
U=\frac{1}{2}\left(x_{0}^{2}+y_{0}^{2}\right)+\frac{1-\mu}{r_{1}}+\frac{\mu}{r_{2}} .
$$

Taking the first derivative of $U$ with respect to $x_{0}$, the $x$-position of the satellite,

$$
\frac{\partial U}{\partial x_{0}}=\frac{1}{2} 2 x_{0}+(1-\mu) \frac{\partial r_{1}^{-1}}{\partial x_{0}}+\mu \frac{\partial r_{2}^{-1}}{\partial x_{0}} .
$$


The magnitudes of $r_{1}$ and $r_{2}$ are

$$
\begin{aligned}
& r_{1}=\left[\left(x_{1}-x_{0}\right)^{2}+\left(y_{1}-y_{0}\right)^{2}\right]^{-\frac{1}{2}} \\
& r_{2}=\left[\left(x_{2}-x_{0}\right)^{2}+\left(y_{2}-y_{0}\right)^{2}\right]^{-\frac{1}{2}} .
\end{aligned}
$$

Therefore,

$$
\begin{aligned}
& \frac{\partial r_{1}^{-1}}{\partial x_{0}}=-\frac{1}{2}\left[\left(x_{1}-x_{0}\right)^{2}+\left(y_{1}-y_{0}\right)^{2}\right]^{-\frac{3}{2}} 2\left(x_{1}-x_{0}\right)(-1)=\frac{\left(x_{1}-x_{0}\right)}{r_{1}^{3}} \\
& \frac{\partial r_{2}^{-1}}{\partial x_{0}}=-\frac{1}{2}\left[\left(x_{2}-x_{0}\right)^{2}+\left(y_{2}-y_{0}\right)^{2}\right]^{-\frac{3}{2}} 2\left(x_{2}-x_{0}\right)(-1)=\frac{\left(x_{2}-x_{0}\right)}{r_{1}^{3}} .
\end{aligned}
$$

Thus,

$$
\frac{\partial U}{\partial x_{0}}=x_{0}+(1-\mu) \frac{\left(x_{1}-x_{0}\right)}{r_{1}^{3}}+\mu \frac{\left(x_{2}-x_{0}\right)}{r_{2}^{3}}
$$

Similarly,

$$
\frac{\partial U}{\partial y_{0}}=y_{0}+(1-\mu) \frac{\left(y_{1}-y_{0}\right)}{r_{1}^{3}}+\mu \frac{\left(y_{2}-y_{0}\right)}{r_{2}^{3}} .
$$

Equations 4.11 and 4.12 can be written in the form

$$
\begin{aligned}
& \ddot{x}_{0}-2 \dot{y_{0}}=x_{0}+\frac{(1-\mu)\left(x_{1}-x_{0}\right)}{r_{1}^{3}}+\frac{\mu\left(x_{2}-x_{0}\right)}{r_{2}^{3}}, \\
& \ddot{y_{0}}+2 \dot{x_{0}}=y_{0}+\frac{(1-\mu)\left(y_{1}-y_{0}\right)}{r_{1}^{3}}+\frac{\mu\left(y_{2}-y_{0}\right)}{r_{2}^{3}} .
\end{aligned}
$$

Using the expressions for $\frac{\partial U}{\partial x_{0}}$ and $\frac{\partial U}{\partial y_{0}}$ we obtain,

$$
\begin{aligned}
& \ddot{x}_{0}-2 \dot{y}_{0}=\frac{\partial U}{\partial x_{0}}, \\
& \ddot{y}_{0}+2 \dot{x}_{0}=\frac{\partial U}{\partial y_{0}} .
\end{aligned}
$$

Now the two equations of motion are in terms of the partial derivatives of the Pseudo-Potential. 


\subsection{Surfaces of Zero Velocity}

We will now show that the kinetic energy of the satellite determines the region of space which is accessible to it. The bounding surface of this region of space is called a surface of zero velocity. Let us begin by multiplying Equation 4.14 by $\dot{x}_{0}$ and Equation 4.15 by $\dot{y}_{0}$ to obtain the following equations:

$$
\begin{aligned}
& \ddot{x}_{0} \dot{x}_{0}-2 \dot{y}_{0} \dot{x}_{0}=\frac{\partial U}{\partial x_{0}} \dot{x}_{0}, \\
& \ddot{y}_{0} \dot{y}_{0}+2 \dot{x}_{0} \dot{y}_{0}=\frac{\partial U}{\partial y_{0}} \dot{y}_{0} .
\end{aligned}
$$

Adding these two equations yields

$$
\ddot{x}_{0} \dot{x}_{0}+\ddot{y}_{0} \dot{y}_{0}=\frac{\partial U}{\partial x_{0}} \dot{x}_{0}+\frac{\partial U}{\partial y_{0}} \dot{y}_{0}=\frac{\partial U}{\partial x_{0}} \frac{d x_{0}}{d t}+\frac{\partial U}{\partial y_{0}} \frac{d y_{0}}{d t} .
$$

Since $\mathrm{U}$ is a function of $x_{0}$ and $y_{0}$,

$$
\frac{d U}{d t}=\frac{\partial U}{\partial x_{0}} \frac{d x_{0}}{d t}+\frac{\partial U}{\partial y_{0}} \frac{d y_{0}}{d t},
$$

and

$$
\begin{aligned}
& \ddot{x}_{0} \dot{x}_{0}=\frac{1}{2} \frac{d}{d t} \dot{x}_{0}^{2}, \\
& \ddot{y}_{0} \dot{y}_{0}=\frac{1}{2} \frac{d}{d t} \dot{y}_{0}^{2} .
\end{aligned}
$$

The expression becomes,

$$
\frac{1}{2} \frac{d}{d t} \dot{x}_{0}^{2}+\frac{1}{2} \frac{d}{d t} \dot{y}_{0}^{2}=\frac{d U}{d t} .
$$

Multiplying through by $d t$,

$$
\frac{1}{2} d \dot{x}_{0}^{2}+\frac{1}{2} d \dot{y}_{0}^{2}=d U
$$

so

$$
\frac{1}{2} \int d \dot{x}_{0}^{2}+\frac{1}{2} \int d \dot{y}_{0}^{2}=\int d U
$$




$$
\begin{gathered}
\frac{1}{2}\left(\dot{x}_{0}^{2}+\dot{y}_{0}^{2}\right)=U-\frac{C}{2}, \\
\dot{x}_{0}^{2}+\dot{y}_{0}^{2}=2 U-C .
\end{gathered}
$$

The left hand side is the square of the velocity, so let us rewrite the equation as follows:

$$
v_{0}^{2}=2 U-C
$$

Using Equation 4.13 for $U$ we obtain,

$$
v_{0}^{2}=x_{0}^{2}+y_{0}^{2}+\frac{2(1-\mu)}{r_{1}}+\frac{2 \mu}{r_{2}}-C .
$$

(Recall that we have scaled $G$ to unity by our choice of units.) If we take the satellite's velocity to be zero,

$$
x_{0}^{2}+y_{0}^{2}+\frac{2(1-\mu)}{r_{1}}+\frac{2 \mu}{r_{2}}=C .
$$

The region bounded by the surface of zero velocity defines where the satellite may be found for a given value of $C$. This surface is known as Hill's limiting surface (Danby, 1962). Regions of space for which $2 U>C$ would give negative values for $v_{0}^{2}$ and therefore imaginary values for $v_{0}$. Such regions are not accessible to the satellite.

For each value of $C$ there are two surfaces of zero velocity, one about each massive body. If two of these surfaces intersect at some point, then each of these is called a Hill sphere and bounds a region of space where the gravitational influence of the enclosed body dominates the attraction of satellites. See Figure 9.

Note that the point where the Earth's Hill sphere and the Moon's Hill sphere intersect is a point of gravitational equilibrium. Note also that it lies directly on a line between the Earth and the Moon. This is the location of one of the Lagrange points, $L_{1}$. As shown in Section 3.1.6 there are three Lagrange points on the line 


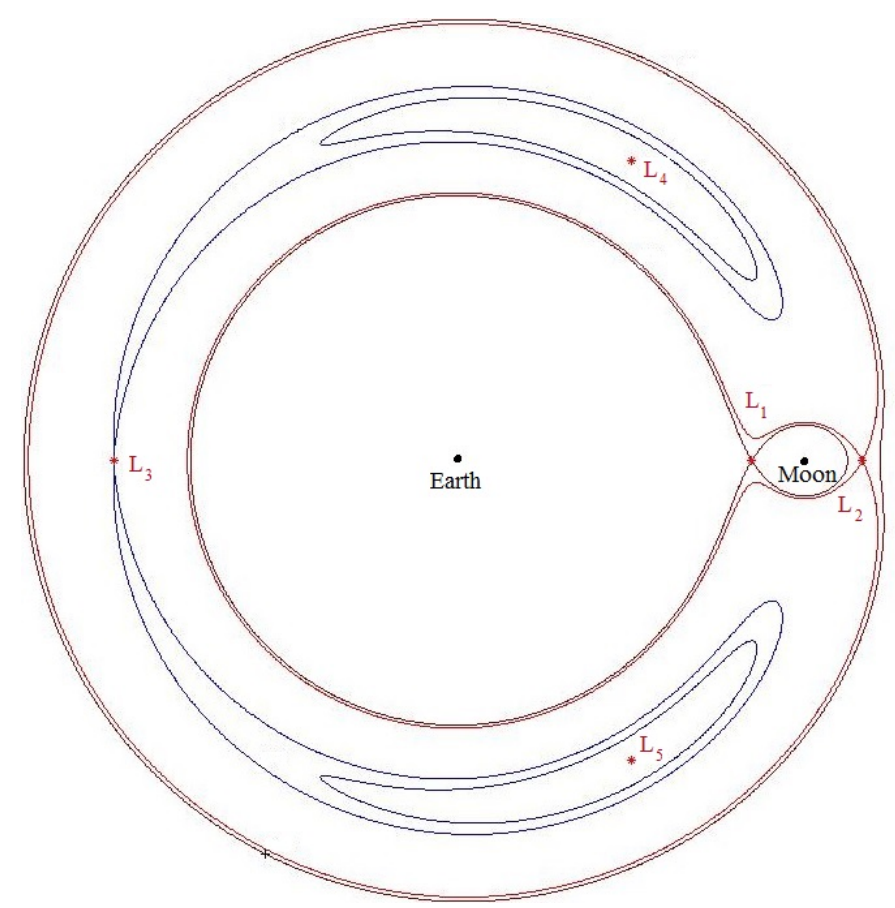

Figure 9. Surfaces of zero velocity. Note that the surfaces about the primary (Earth) and secondary (Moon) which intersect are the Hill Spheres about these masses.

through the Earth and the Moon, one each on the far sides of the Earth and the Moon.

\subsection{Analysis}

Recall that the Lagrange points are equilibrium points. Therefore, the acceleration and velocity of a massive body at one of the Lagrange points should be zero in the rotating frame. Consequently, from Equations 4.14 and 4.15, the partial derivatives of $\mathrm{U}$ with respect to $x_{0}$ and $y_{0}$ evaluated at the Lagrange point must be zero. Let us call the location of one of these Lagrange points $\left(x_{L}, y_{L}\right)$. Let the particle be displaced to $\left(x_{L}+X, y_{L}+Y\right)$ where $X, Y$ are small. Assume the particle has a velocity $\vec{v}$,

$$
\vec{v}=\dot{X} \hat{x}+\dot{Y} \hat{y}
$$


Recall that the form of a Taylor series expansion of a function $f(x, y)$ about a point $(a, b)$ in two dimensions is

$$
f(x, y)=f(a, b)+\left[\frac{\partial f}{\partial x}\right]_{a, b}(x-a)+\left[\frac{d f}{d y}\right]_{a, b}(y-b)+\frac{1}{2 !}\left[\frac{\partial^{2} f}{\partial x^{2}}\right]_{a, b}(x-a)^{2}+\ldots
$$

Let us perform a Taylor series expansion on the right hand side of equation 4.14 (that is, we will Taylor expand $\frac{\partial U}{\partial x_{0}}$ ). Note that since the expansion is taken about the equilibrium point, the first term will be zero. Let us ignore the terms involving squares and higher power coefficients of the displacement, as they will be much smaller than the displacement itself, which is small to begin with. Taking the Taylor series expansion,

$$
\begin{gathered}
\ddot{x}_{0}-2 \dot{y}_{0}=\left(\frac{\partial U}{\partial x_{0}}\right)_{x_{L}, y_{L}}+\left(X-x_{L}\right) \frac{\partial}{\partial x_{0}}\left(\frac{\partial U}{\partial x_{0}}\right)_{x_{L}, y_{L}} \\
+\left(Y-y_{L}\right) \frac{\partial}{\partial y_{0}}\left(\frac{\partial U}{\partial x_{0}}\right)_{x_{L}, y_{L}}+\mathcal{O}\left(X^{2}, Y^{2}\right) .
\end{gathered}
$$

The first term on the right hand side is zero since the derivative is evaluated at the Lagrange point, which is an equilibrium point. Ignoring second and higher order terms, the Taylor series expansion yields

$$
\ddot{x}_{0}-2 \dot{y}_{0}=X\left(\frac{\partial^{2} U}{\partial x_{0}^{2}}\right)_{x_{L}, y_{L}}+Y\left(\frac{\partial^{2} U}{\partial x_{0} \partial y_{0}}\right)_{x_{L}, y_{L}} .
$$

Performing a similar expansion on equation 4.15 ,

$$
\ddot{y}_{0}+2 \dot{x}_{0}=X\left(\frac{\partial^{2} U}{\partial x_{0} \partial y_{0}}\right)_{x_{L}, y_{L}}+Y\left(\frac{\partial^{2} U}{\partial y_{0}^{2}}\right)_{x_{L}, y_{L}} .
$$

These equations are second order, coupled, linear differential equations with constant coefficients. It is well known that these differential equations have four independent solutions of the form: 


$$
\begin{aligned}
& X=\sum_{i=1}^{4} \alpha_{i} e^{\lambda_{i} t} \\
& Y=\sum_{i=1}^{4} \beta_{i} e^{\lambda_{i} t} .
\end{aligned}
$$

All of these are solutions. Therefore, each one is a solution. We consider a single solution

$$
X=\alpha e^{\lambda t}
$$

Differentiating,

$$
\begin{aligned}
& \dot{X}=\alpha \lambda e^{\lambda t}, \\
& \ddot{X}=\alpha \lambda^{2} e^{\lambda t} .
\end{aligned}
$$

Since $\ddot{x}_{0}=\ddot{X}$, we can rewrite Equation 4.16 as,

$$
\ddot{X}-2 \dot{Y}=X \frac{\partial^{2} U}{\partial x_{0}^{2}}+Y \frac{\partial^{2} U}{\partial x_{0} \partial y_{0}}
$$

or,

$$
\left(\frac{d^{2}}{d t^{2}}-\frac{\partial^{2} U}{\partial x_{0}^{2}}\right) X-\left(2 \frac{d}{d t}-\frac{\partial^{2} U}{\partial x_{0} \partial y_{0}}\right) Y=0
$$

Similarly, for Equation 4.17,

$$
\left(\frac{d^{2}}{d t^{2}}-\frac{\partial^{2} U}{\partial y_{0}^{2}}\right) Y+\left(2 \frac{d}{d t}-\frac{\partial^{2} U}{\partial x_{0} \partial y_{0}}\right) X=0
$$

Since we have two equations of the forms:

$$
\begin{aligned}
& A X+B Y=C, \\
& D X+E Y=F,
\end{aligned}
$$


the value of $X$ can be determined by Cramer's rule,

$$
X=\frac{\left|\begin{array}{cc}
C & B \\
F & E
\end{array}\right|}{\left|\begin{array}{ll}
A & B \\
D & E
\end{array}\right|}
$$

Since $C=F=0$,

$$
X=\frac{\left|\begin{array}{ll}
0 & B \\
0 & E
\end{array}\right|}{\left|\begin{array}{cc}
A & B \\
D & E
\end{array}\right|}=0
$$

This yields the trivial solution $X=0, Y=0$. However, there still remains a possibility for a nontrivial solution. If the determinant in the denominator is also zero, then the expression is undefined and can still be a solution. In other words, a nontrivial solution is obtained if

$$
\begin{gathered}
\left|\begin{array}{cc}
A & B \\
D & E
\end{array}\right|=0, \\
\left|\begin{array}{cc}
\left(\frac{d^{2}}{d t^{2}}-\frac{\partial^{2} U}{\partial x_{0}^{2}}\right) X & \left(-2 \frac{d}{d t}+\frac{\partial^{2} U}{\partial x_{0} \partial y_{0}}\right) Y \\
\left(2 \frac{d}{d t}-\frac{\partial^{2} U}{\partial x_{0} \partial y_{0}}\right) X & \left(\frac{d^{2}}{d t^{2}}-\frac{\partial^{2} U}{\partial y_{0}^{2}}\right) Y
\end{array}\right|=0 .
\end{gathered}
$$

Let us examine the following term,

$$
\left(\frac{d^{2}}{d t^{2}}-\frac{\partial^{2} U}{\partial x_{0}^{2}}\right) X
$$

By equation 4.18,

$$
\begin{aligned}
& \left(\frac{d^{2}}{d t^{2}}-\frac{\partial^{2} U}{\partial x_{0}^{2}}\right) X=\left(\frac{d^{2}}{d t^{2}}-\frac{\partial^{2} U}{\partial x_{0}^{2}}\right) \alpha e^{\lambda t} \\
& \left(\frac{d^{2}}{d t^{2}}-\frac{\partial^{2} U}{\partial x_{0}^{2}}\right) X=\alpha \lambda^{2} e^{\lambda t}-\frac{\partial^{2} U}{\partial x_{0}^{2}} \alpha e^{\lambda t} \\
& \left(\frac{d^{2}}{d t^{2}}-\frac{\partial^{2} U}{\partial x_{0}^{2}}\right) X=\alpha e^{\lambda t}\left(\lambda^{2}-\frac{\partial^{2} U}{\partial x_{0}^{2}}\right) .
\end{aligned}
$$


Since $X=\alpha e^{\lambda t}$

$$
\left(\frac{d^{2}}{d t^{2}}-\frac{\partial^{2} U}{\partial x_{0}^{2}}\right) X=\left(\lambda^{2}-\frac{\partial^{2} U}{\partial x_{0}^{2}}\right) X
$$

It can be shown similarly that

$$
\begin{aligned}
\left(\frac{d^{2}}{d t^{2}}-\frac{\partial^{2} U}{\partial y_{0}^{2}}\right) Y & =\left(\lambda^{2}-\frac{\partial^{2} U}{\partial y_{0}^{2}}\right) Y \\
\left(-2 \frac{d}{d t}+\frac{\partial^{2} U}{\partial x_{0} \partial y_{0}}\right) Y & =\left(-2 \lambda+\frac{\partial^{2} U}{\partial x_{0} \partial y_{0}}\right) Y \\
\left(2 \frac{d}{d t}-\frac{\partial^{2} U}{\partial x_{0} \partial y_{0}}\right) X & =\left(2 \lambda-\frac{\partial^{2} U}{\partial x_{0} \partial y_{0}}\right) X
\end{aligned}
$$

and our characteristic determinant becomes

$$
\left|\begin{array}{cc}
\alpha e^{\lambda t}\left(\lambda^{2}-\frac{\partial^{2} U}{\partial x_{0}^{2}}\right) & \beta e^{\lambda t}\left(-2 \lambda+\frac{\partial^{2} U}{\partial x_{0} \partial y_{0}}\right) \\
\alpha e^{\lambda t}\left(2 \lambda-\frac{\partial^{2} U}{\partial x_{0} \partial y_{0}}\right) & \beta e^{\lambda t}\left(\lambda^{2}-\frac{\partial^{2} U}{\partial y_{0}^{2}}\right)
\end{array}\right|=0 .
$$

Note that the $e^{\lambda t}$ is a common non-zero factor. Therefore, we can remove it altogether from our expression without any effect on the validity of the determinant.

$$
\left|\begin{array}{cc}
\alpha\left(\lambda^{2}-\frac{\partial^{2} U}{\partial x_{0}^{2}}\right) & \beta\left(-2 \lambda+\frac{\partial^{2} U}{\partial x_{0} \partial y_{0}}\right) \\
\alpha\left(2 \lambda-\frac{\partial^{2} U}{\partial x_{0} \partial y_{0}}\right) & \beta\left(\lambda^{2}-\frac{\partial^{2} U}{\partial y_{0}^{2}}\right)
\end{array}\right|=0 .
$$

Our characteristic equation becomes

$$
\alpha \beta\left(\lambda^{2}-\frac{\partial^{2} U}{\partial x_{0}^{2}}\right)\left(\lambda^{2}-\frac{\partial^{2} U}{\partial y_{0}^{2}}\right)-\alpha \beta\left(-2 \lambda+\frac{\partial^{2} U}{\partial x_{0} \partial y_{0}}\right)\left(2 \lambda-\frac{\partial^{2} U}{\partial x_{0} \partial y_{0}}\right)=0
$$

Note that $\alpha \beta$ is also a nonzero common factor that can be removed while the equation remains true. Thus, our characteristic equation becomes

$$
\begin{gathered}
\left(\lambda^{2}-\frac{\partial^{2} U}{\partial x_{0}^{2}}\right)\left(\lambda^{2}-\frac{\partial^{2} U}{\partial y_{0}^{2}}\right)-\left(-2 \lambda+\frac{\partial^{2} U}{\partial x_{0} \partial y_{0}}\right)\left(2 \lambda-\frac{\partial^{2} U}{\partial x_{0} \partial y_{0}}\right)=0 . \\
\lambda^{4}+\left(4-\frac{\partial^{2} U}{\partial x_{0}^{2}}-\frac{\partial^{2} U}{\partial y_{0}^{2}}\right) \lambda^{2}+\frac{\partial^{2} U}{\partial x_{0}^{2}} \frac{\partial^{2} U}{\partial y_{0}^{2}}-\left(\frac{\partial^{2} U}{\partial x_{0} \partial y_{0}}\right)^{2}=0
\end{gathered}
$$


If all the $\lambda$ 's are real, then the positions associated with the exponential terms containing $\lambda$ 's will increase with time, and the satellite will drift away from the equilibrium point. However, if the $\lambda$ 's are imaginary, the position of the satellite will oscillate about the equilibrium point; the equilibrium will be stable. We will need to evaluate the second partial derivatives of $U$ in order to determine if the $\lambda$ 's are real or imaginary. (See Section 4.6.)

$$
\frac{\partial^{2} U}{\partial x_{0}^{2}}=\frac{\partial}{\partial x_{0}} \frac{\partial U}{\partial x_{0}}=\frac{\partial}{\partial x_{0}}\left[x_{0}+(1-\mu) \frac{\left(x_{1}-x_{0}\right)}{r_{1}^{3}}+\mu \frac{\left(x_{2}-x_{0}\right)}{r_{2}^{3}}\right] .
$$

Considering the middle term of the right hand side,

$$
\begin{gathered}
\frac{\partial}{\partial x_{0}}(1-\mu) \frac{\left(x_{1}-x_{0}\right)}{r_{1}^{3}}=(1-\mu)\left[\frac{1}{r_{1}^{3}} \frac{\partial}{\partial x_{0}}\left(x_{1}-x_{0}\right)+\left(x_{1}-x_{0}\right) \frac{\partial}{\partial x_{0}} \frac{1}{r_{1}^{3}}\right] \\
\frac{\partial}{\partial x_{0}} \frac{1}{r_{1}^{3}}=\frac{\partial}{\partial x_{0}}\left[\left(x_{1}-x_{0}\right)^{2}+\left(y_{1}-y_{0}\right)^{2}\right]^{-\frac{3}{2}} \\
\frac{\partial}{\partial x_{0}} \frac{1}{r_{1}^{3}}=-\frac{3}{2}\left[\left(x_{1}-x_{0}\right)^{2}+\left(y_{1}-y_{0}\right)^{2}\right]^{-\frac{5}{2}} 2\left(x_{1}-x_{0}\right)(-1)=\frac{3\left(x_{1}-x_{0}\right)}{r_{1}^{5}} \\
\frac{\partial^{2} U}{\partial x_{0}^{2}}=1-\frac{(1-\mu)}{r_{1}^{3}}-\frac{\mu}{r_{2}^{3}}+3(1-\mu) \frac{\left(x_{1}-x_{0}\right)^{2}}{r_{1}^{5}}+3 \mu \frac{\left(x_{2}-x_{0}\right)^{2}}{r_{2}^{5}} .
\end{gathered}
$$

Similarly,

$$
\frac{\partial^{2} U}{\partial y_{0}^{2}}=1-\frac{(1-\mu)}{r_{1}^{3}}-\frac{\mu}{r_{2}^{3}}+3(1-\mu) \frac{\left(y_{1}-y_{0}\right)^{2}}{r_{1}^{5}}+3 \mu \frac{\left(y_{2}-y_{0}\right)^{2}}{r_{2}^{5}}
$$

Furthermore, we can express the mixed partial derivative as

$$
\frac{\partial}{\partial y_{0}}\left(\frac{\partial U}{\partial x_{0}}\right)=\frac{\partial}{\partial y_{0}}\left[x_{0}+(1-\mu) \frac{\left(x_{1}-x_{0}\right)}{r_{1}^{3}}+\mu \frac{\left(x_{2}-x_{0}\right)}{r_{2}^{3}}\right] \text {. }
$$


The first term is zero since $\frac{\partial x_{0}}{\partial y_{0}}=0$. The second term is

$$
\frac{\partial}{\partial y_{0}}\left[\frac{-(1-\mu)\left(x_{1}-x_{0}\right)}{r_{1}^{3}}\right]=-(1-\mu)\left(x_{1}-x_{0}\right) \frac{\partial}{\partial y_{0}} \frac{1}{r_{1}^{3}},
$$

because

$$
\frac{\partial r_{1}^{-3}}{\partial y_{0}}=\frac{3\left(y_{1}-y_{0}\right)}{r_{1}^{5}}
$$

Thus,

$$
\frac{\partial^{2} U}{\partial x_{0} \partial y_{0}}=-3\left[\frac{(1-\mu)\left(x_{1}-x_{0}\right)\left(y_{1}-y_{0}\right)}{r_{1}^{5}}+\frac{\mu\left(x_{2}-x_{0}\right)\left(y_{2}-y_{0}\right)}{r_{2}^{5}}\right] \text {. }
$$

Now we have expressions for all three second derivatives of the

Pseudo-Potential. We will use these in our Taylor expansions of the equations of motion for the satellite. This will indicate whether the Lagrange points are stable or unstable equilibrium points.

\subsection{The Stability of the Lagrange Points}

Let us now consider the stability of the Lagrange points, beginning with the collinear Lagrange points. For these points, $y_{0}=0$; thus, the $\left(y_{1}-y_{0}\right)$ and $\left(y_{2}-y_{0}\right)$ terms in Equation 4.24 are also zero since $y_{1}=y_{2}=0$. Therefore the mixed partial derivative is zero (see Equation 4.24).

$$
\frac{\partial^{2} U}{\partial x_{0} \partial y_{0}}=0
$$

For convenience, let us define a quantity $A$ given by

$$
A=\frac{1-\mu}{r_{1}^{3}}+\frac{\mu}{r_{2}^{3}}
$$

From Equation 4.22,

$$
\frac{\partial^{2} U}{\partial x_{0}^{2}}=1-A+3(1-\mu) \frac{\left(x_{1}-x_{0}\right)^{2}}{r_{1}^{5}}+3 \mu \frac{\left(x_{2}-x_{0}\right)^{2}}{r_{2}^{5}},
$$


but since $y_{1}=y_{2}=y_{0}=0$,

$$
r_{1}^{2}=\left(x_{1}-x_{0}\right)^{2}
$$

and similarly,

$$
r_{2}^{2}=\left(x_{2}-x_{0}\right)^{2},
$$

therefore,

$$
\frac{\partial^{2} U}{\partial x_{0}^{2}}=1-A+3(1-\mu) \frac{1}{r_{1}^{3}}+3 \mu \frac{1}{r_{2}^{3}}=1-A+3 A=1+2 A .
$$

Due to the same condition on the $y$-coordinates for the collinear Lagrange points, from Equation 4.23

$$
\frac{\partial^{2} U}{\partial y_{0}^{2}}=1-A
$$

Therefore Equation 4.21 becomes

$$
\begin{gathered}
\lambda^{4}+[4-(1+2 A)-(1-A)] \lambda^{2}+(1+2 A)(1-A)=0, \\
\lambda^{4}+(2-A) \lambda^{2}+\left(1+A-2 A^{2}\right)=0 .
\end{gathered}
$$

Solving the quadratic in $\lambda^{2}$,

$$
\lambda^{2}=\frac{-(2-A) \pm \sqrt{(2-A)^{2}-4(1)\left(1+A-2 A^{2}\right)}}{2} .
$$

Recall from Section 4.5 that whether $\lambda$ has real or imaginary roots at a Lagrange point governs whether the Lagrange point is a stable equilibrium or not. The solutions have the forms

$$
\begin{aligned}
& X=\sum_{i=1}^{4} \alpha_{i} e^{\lambda_{i} t}, \\
& Y=\sum_{i=1}^{4} \beta_{i} e^{\lambda_{i} t} .
\end{aligned}
$$


Recall again that if any of the $\lambda^{\prime} s$ are not imaginary numbers, the particle will drift away from the equilibrium point to infinity. A stable equilibrium point requires that all of the $\lambda^{\prime} s$ be imaginary.

In order for $\lambda$ to be real or complex, the term under the radical must be greater than $-(2-A)$. The quantity under the radical is

$$
(2-A)^{2}-4\left(1+A-2 A^{2}\right)=-8 A+9 A^{2} .
$$

That is, for real roots (unstable equilibrium points) the following condition must be met

$$
\left[-8 A+9 A^{2}\right]^{\frac{1}{2}}>-(2-A)
$$

or,

$$
2 A^{2}-A>1
$$

We will now determine the distances from the primary and secondary masses to the collinear Lagrange points. The position of the collinear Lagrange points can be found from the fact that the pseudo-potential is an extremum at equilibrium points (see section 4.5). Thus, the partial derivatives of the pesudo-potential (see Equation 4.13) must be zero at these points.

$$
\begin{aligned}
& \frac{\partial U}{\partial x_{0}}=x_{0}+(1-\mu) \frac{\left(x_{1}-x_{0}\right)}{r_{1}^{3}}+\mu \frac{\left(x_{2}-x_{0}\right)}{r_{2}^{3}}=0, \\
& \frac{\partial U}{\partial y_{0}}=y_{0}+(1-\mu) \frac{\left(y_{1}-y_{0}\right)}{r_{1}^{3}}+\mu \frac{\left(y_{2}-y_{0}\right)}{r_{2}^{3}}=0 .
\end{aligned}
$$

Since $x_{0}$ is the distance (see Figure 10 below) from the center of mass to the equilibrium point, we have the following relations

$$
\begin{gathered}
r_{1}^{2}=\left(\mu+x_{0}\right)^{2}, \\
r_{2}^{2}=\left[(1-\mu)-x_{0}\right]^{2},
\end{gathered}
$$


and our expression for $\frac{\partial U}{\partial x_{0}}$ becomes

$$
\begin{gathered}
\frac{\partial U}{\partial x_{0}}=x_{0}+(1-\mu) \frac{r_{1}}{r_{1}^{3}}+\mu \frac{r_{2}}{r_{2}^{3}}, \\
\frac{\partial U}{\partial x_{0}}=x_{0}+(1-\mu) \frac{1}{r_{1}^{2}}+\mu \frac{1}{r_{2}^{2}}, \\
\frac{\partial U}{\partial x_{0}}=x_{0}+\frac{1-\mu}{\left(-\mu-x_{0}\right)^{2}}+\frac{\mu}{\left[(1-\mu)-x_{0}\right]^{2}}=0 .
\end{gathered}
$$

Murray and Dermott (1999) use this condition to determine the positions of the three collinear points. Their equation is

$$
\frac{\mu}{1-\mu}=3 r_{2}^{3} \frac{1-r_{2}+\frac{1}{3} r_{2}^{2}}{\left(1+r_{2}+r_{2}^{2}\right)\left(1-r_{2}\right)^{3}} .
$$

Murray and Dermott give approximate solutions to this equation for the Lagrange points.

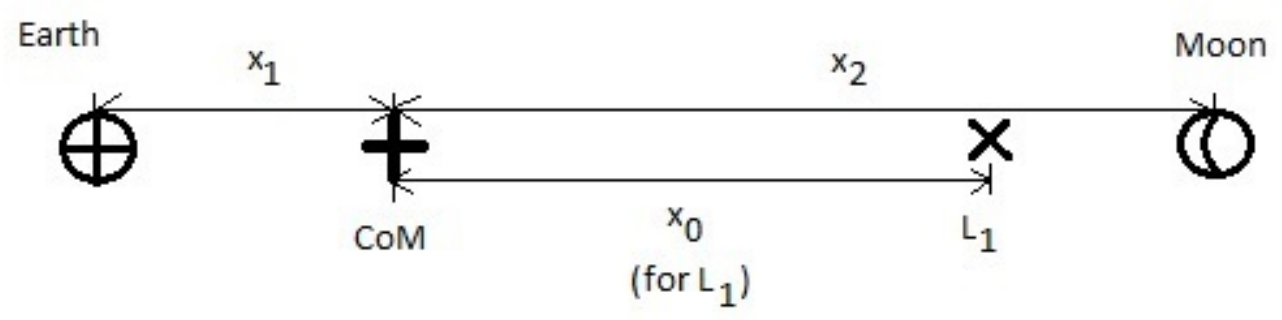

Figure 10. The distances $x_{1}, x_{2}$, and $x_{0}$ are taken with respect to the center of mass. (The $L_{1}$ case is shown.)

Cornish (2011) used a different technique to determine the location of the collinear Lagrange points, subject to the condition that $1-\mu \gg \mu$. Cornish's approximations lead to $L_{2}$ being the same distance from the secondary mass (the Moon) as $L_{1}$. However, it is well known that $L_{2}$ is further from the Moon than $L_{1}$. 
Nevertheless, this approximation has no effect on whether or not $\lambda$ 's are pure imaginaries (that is, whether the collinear Lagrange points are stable or not).

We shall examine all three, beginning with $L_{1}$. Assume the satellite is placed at $L_{1}$. According to Cornish, the distance from the Moon to $L_{1}$ is

$$
r_{2}=r \cdot \sqrt[3]{\frac{\mu}{3}},
$$

where $r$ is the (average) separation between the Earth and the Moon.

Thus it follows that the distance from the Earth to $L_{1}$ is

$$
r_{1}=r\left(1-\sqrt[3]{\frac{\mu}{3}}\right) .
$$

For convenience, let us define a quantity $\nu$ by

$$
\nu^{3}=\frac{\mu}{3} .
$$

Cubing Equations 4.28 and 4.29,

$$
\begin{gathered}
r_{2}^{3}=(r \nu)^{3}=r^{3} \nu^{3}, \\
r_{1}^{3}=[r(1-\nu)]^{3}=r^{3}-3 r^{3} \nu+3 r^{3} \nu^{2}-r^{3} \nu^{3} .
\end{gathered}
$$

The mass of the Moon is $1.23 \%$ the mass of the Earth, so $\nu^{3}$ is $0.405 \%$. Thus, $\nu$ is $15.9 \%$. Therefore from Equation 4.29,

$$
r_{1} \approx(0.84) r .
$$

Similarly for $r_{2}$,

$$
r_{2} \approx(0.16) r .
$$

This shows that $L_{1}$ lies between the Earth and the Moon, much closer to the Moon than the Earth.

Inserting Equations 4.28 and 4.29 into Equation 4.25,

$$
A=\frac{1-\mu}{r_{1}^{3}}+\frac{\mu}{r_{2}^{3}},
$$




$$
\begin{aligned}
& A=\frac{1-\mu}{\left[r\left(1-\sqrt[3]{\frac{\mu}{3}}\right)\right]^{3}}+\frac{\mu}{r^{3} \frac{\mu}{3}}, \\
& A=\frac{1-\mu}{\left[r\left(1-\sqrt[3]{\frac{\mu}{3}}\right)\right]^{3}}+\frac{3}{r^{3}}
\end{aligned}
$$

Inserting this expression for $A$ into Equation 4.27 yields

$$
2 A^{2}-A=2\left[\frac{1-\mu}{\left[r\left(1-\sqrt[3]{\frac{\mu}{3}}\right)\right]^{3}}+\frac{3}{r^{3}}\right]^{2}-\left[\frac{1-\mu}{\left[r\left(1-\sqrt[3]{\frac{\mu}{3}}\right)\right]^{3}}+\frac{3}{R^{3}}\right]>1
$$

Using these values for $A$ and $A^{2}$, it can be shown for $L_{1}$ that for any $\mu$ such that

$$
0<\mu \leq \frac{1}{2}
$$

our condition in Equation 4.27 will be met. Therefore none of the $\lambda$ 's are pure imaginaries at $L_{1}$. Thus, $L_{1}$ is an unstable equilibrium point.

Let us examine now the equilibrium for Lagrange point $L_{2}$. Now the satellite is at $L_{2}$. Cornish (2011) shows that the distance from the Moon to $L_{2}$ is

$$
r_{2}=r \cdot \sqrt[3]{\frac{\mu}{3}}
$$

Thus, the distance from the Earth to $L_{2}$ is

$$
r_{1}=r\left(1+\sqrt[3]{\frac{\mu}{3}}\right)
$$

The value for $r_{1}^{3}$ for $L_{2}$ is then

$$
r_{1}^{3}=\left[r\left(1+\sqrt[3]{\frac{\mu}{3}}\right)\right]^{3} .
$$

Thus our expression for $A$ from Equation 4.25 becomes:

$$
A=\frac{1-\mu}{\left[r\left(1+\sqrt[3]{\frac{\mu}{3}}\right)\right]^{3}}+\frac{\mu}{r_{2}^{3}}
$$




$$
\begin{aligned}
& A=\frac{(1-\mu)}{\left[r\left(1+\sqrt[3]{\frac{\mu}{3}}\right)\right]^{3}}+\frac{\mu}{r^{3} \frac{\mu}{3}}, \\
& A=\frac{(1-\mu)}{\left[r\left(1+\sqrt[3]{\frac{\mu}{3}}\right)\right]^{3}}+\frac{3}{r^{3}} .
\end{aligned}
$$

Note that this is a similar result to $L_{1}$, and the condition on $A$ (Equation 4.27 ) is still met for $0<\mu \leq \frac{1}{2}$. Thus none of the $\lambda$ 's are pure imaginaries, and $L_{2}$ is also an unstable equilibrium point.

Let us now examine $L_{3}$. Placing the satellite at $L_{3}$, the distance from the Moon to the satellite is $r_{2}$. Cornish (2011) showed that the distance from the Earth to $L_{3}$ is

$$
r_{1}=r\left(1+\frac{5 \mu}{12}\right)
$$

Therefore,

$$
r_{2}=r\left(2+\frac{5 \mu}{12}\right) .
$$

Inserting Equations 4.39 and 4.40 into 4.25,

$$
A=\frac{1-\mu}{\left[r\left(1+\frac{5 \mu}{12}\right)\right]^{3}}+\frac{\mu}{\left[r\left(2+\frac{5 \mu}{12}\right)\right]^{3}} .
$$

It can be shown that for any $0<\mu \leq \frac{1}{2}$, that the condition on $\lambda$ (Equation $4.27)$ is still met. Therefore none of the $\lambda$ 's are pure imaginaries at $L_{3}$, and the third and final collinear Lagrange point is an unstable equilibrium. Thus, all the collinear Lagrange points are unstable equilibria.

Let us consider the triangular Lagrange points. At these points,

$$
r=r_{1}=r_{2}=1
$$




$$
\begin{aligned}
& x_{0}=\frac{1}{2}-\mu, \\
& y_{0}=\frac{ \pm \sqrt{3}}{2} .
\end{aligned}
$$

Plugging into 4.22,

$$
\frac{\partial^{2} U}{\partial x_{0}^{2}}=\frac{3}{4}
$$

Similarly 4.23 yields

$$
\frac{\partial^{2} U}{\partial y_{0}^{2}}=\frac{9}{4}
$$

and 4.24 gives

$$
\frac{\partial^{2} U}{\partial x_{0} \partial y_{0}}=\frac{3 \sqrt{3}}{4}(1-2 \mu) .
$$

Therefore, Equation 4.21 reduces to

$$
\lambda^{4}+\lambda^{2}+\frac{27}{4} \mu(1-\mu)=0 .
$$

If the all four $\lambda$ 's are imaginary, then the triangular Lagrange points are stable. From Equation 4.41,

$$
\lambda^{2}=\frac{-1 \pm \sqrt{1-27 \mu(1-\mu)}}{2} .
$$

The term under the radical must obey the following condition:

$$
1-27 \mu(1-\mu) \geq 0
$$

Therefore,

$$
\mu \leq \frac{1}{2} \pm \sqrt{\frac{23}{108}} .
$$

This leaves us with two possibilities for the value of $\mu$. Recall that $\mu$ is the ratio of the secondary mass to the total mass, so $\mu$ must be less than $\frac{1}{2}$. Therefore, 
let us take the negative sign in Equation 4.43. This leaves us with the stability condition for the triangular Lagrange points, commonly called the critical mass ratio

$$
\mu<0.0385 \ldots
$$

Therefore, for the orbit of the satellite at one of the triangular Lagrange points to remain stable, the ratio of masses between the secondary to the total mass must be less than this value. If the Moon were more than $3.85 \%$ of the Earth's mass, the triangular Lagrange points of the Earth-Moon system would not be stable equilibrium points, and a third body at one of these points could not maintain a stable orbit.

\subsection{A Satellite With Nonzero Mass}

Lagrange's solution to the circular restricted three-body problem assumes the mass of the satellite is infinitesimal. This led to the critical mass ratio $(\mu)$, an important result. We are interested in exploring the problem of a third body of finite mass. We shall, however, continue to assume that the three bodies are in circular orbits about the center of mass.

In the circular restricted three-body problem (CR3BP), the center of mass is on the line between the primary and secondary masses, a distance $\mu$ from the primary and $(1-\mu)$ from the secondary where the distance between the masses sums to unity. The masses of the primary and secondary also sum to unity since the mass of the primary is $(1-\mu)$ and the mass of the secondary is $\mu$. This means that the $x$-position of the satellite with respect to the center of mass is $\left(\frac{1}{2}-\mu\right)$ and its $y$-position is $\frac{\sqrt{3}}{2}$.

Let us assign a finite mass $\chi$ to the satellite. This brings the total mass of the system to $1+\chi$. Now let us solve for the location of the new center of mass with respect to the classic CR3BP center of mass (see Figures 7 and 11 for diagrams and 
the introduction to Chapter 4 for the coordinates). To begin with, the equation for the $x$ and $y$ coordinates of the center of mass are

$$
\begin{aligned}
& x_{C o M}=\frac{\sum_{i} m_{i} x_{i}}{\sum_{i} m_{i}}, \\
& y_{C o M}=\frac{\sum_{i} m_{i} y_{i}}{\sum_{i} m_{i}} .
\end{aligned}
$$

Solving for the coordinates of the center of mass,

$$
\begin{gathered}
\frac{\sum_{i} m_{i} x_{i}}{\sum_{i} m_{i}}=\frac{(1-\mu)(-\mu)+\mu(1-\mu)+\left(\frac{1}{2}-\mu\right) \chi}{1+\chi}=\frac{\left(\frac{1}{2}-\mu\right) \chi}{1+\chi} \\
\frac{\sum_{i} m_{i} y_{i}}{\sum_{i} m_{i}}=\frac{(\mu) 0+(1-\mu) 0+\frac{\sqrt{3}}{2} \chi}{1+\chi}=\frac{\frac{\sqrt{3}}{2} \chi}{1+\chi}
\end{gathered}
$$

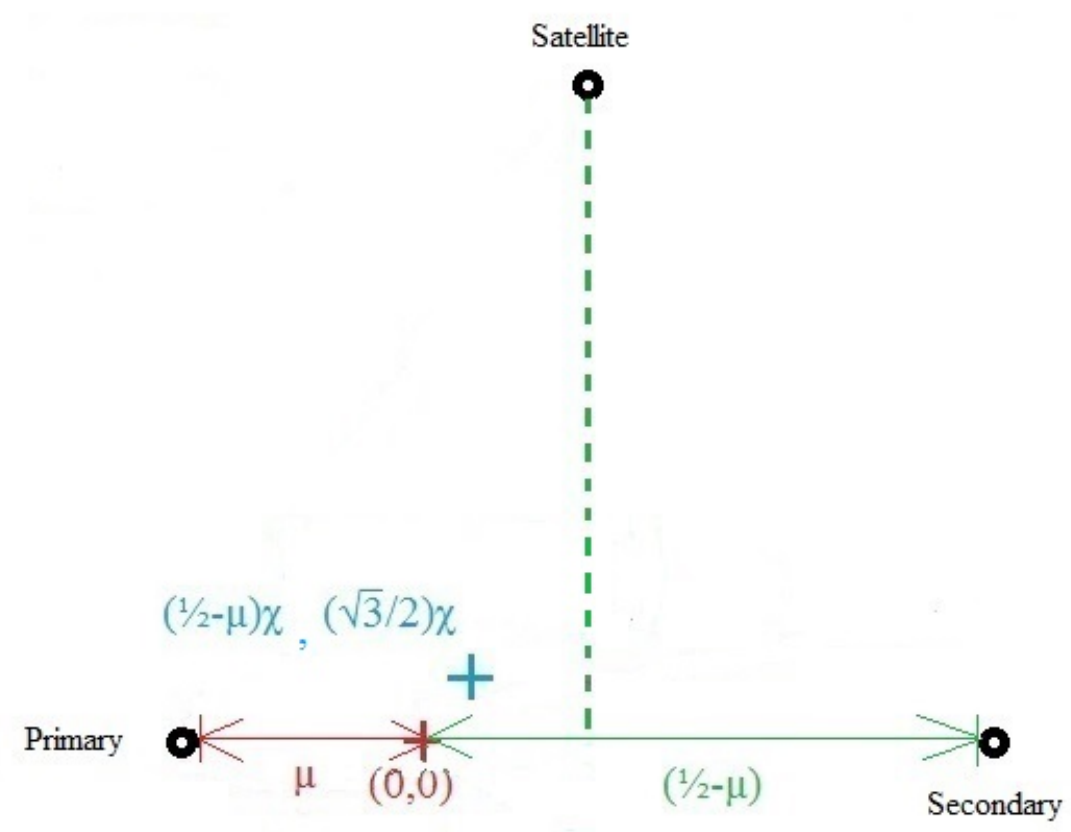

Figure 11. The center of mass for a three-body system with a nonzero mass satellite. The upper cross is the location of the new center of mass, and its coordinates are its position relative to the old center of mass. Note that the old center of mass's coordinates are $(0,0)$. 
We will use a standard coordinate transformation to move from an inertial frame to a rotating frame (see Section 4.1). Let us place the origin at the center of mass of the system. See Figure 12.

Unfortunately, it is not possible to use Lagrange's method to find the mass of the satellite because the mass of the satellite divides out of the equations of motion. The new center of mass depends upon the mass of the satellite $\chi$. It would appear that moving the origin to the new center of mass might lead to new equations of motion involving $\chi$. However, the partial derivatives of the pseudo-potential $U$ involve only the relative distances between the masses (see Equations 4.22, 4.23, and 4.24). Consequently, the quantity $\chi$ does not enter into the stability analysis of the Lagrange points (see Sections 4.5 and 4.6).

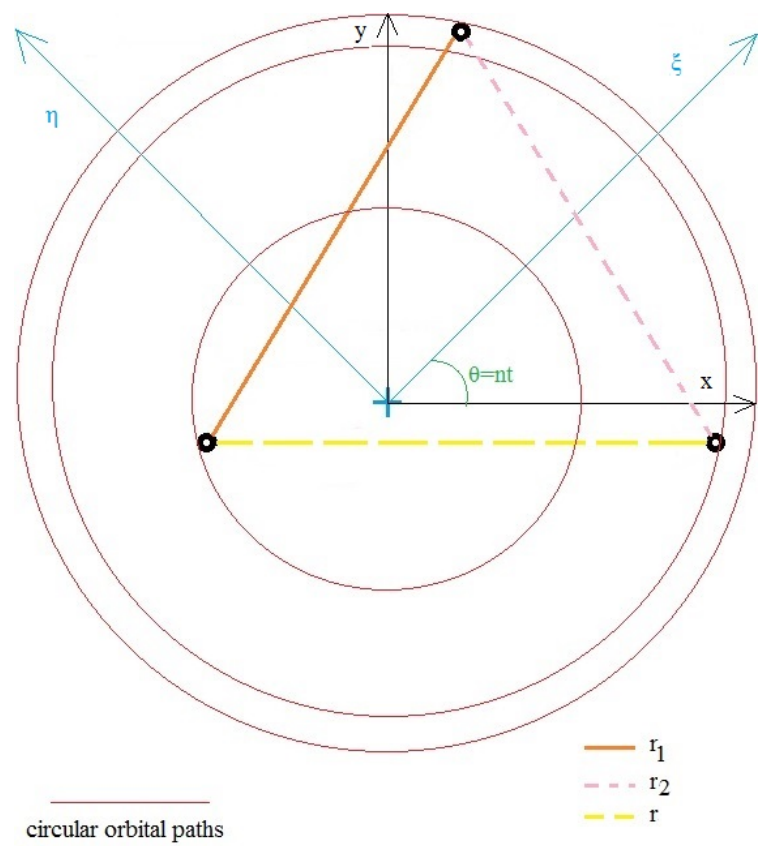

Figure 12. The inertial and corotating frames for the CR3BP with a nonzero mass satellite. $\xi$ and $\eta$ are the inertial axes, $x$ and $y$ are the rotating axes, and the concentric rings are the circular paths that the three masses take as they corotate about the system's center of mass. The relative sizes of the circular paths are not to scale for the Earth-Moon System. 


\section{CHAPTER 5}

\section{THE DISTURBING FUNCTION}

We will now examine the added influence of a third body to a stable two body system by defining a quantity customarily referred to as the disturbing function (Roy, 2005). Recall that $\vec{R}_{1}, \vec{R}_{2}$, and $\vec{R}_{0}$ are the positions of the Earth, Moon, and satellite (respectively) with respect to an inertial origin. Also, $\vec{r}$ and $\vec{r}_{1}$ are the position of the Moon and satellite (respectively) with respect to the Earth. In other words,

$$
\vec{r}_{1}=\vec{R}_{0}-\vec{R}_{1} .
$$

See Figure 13.

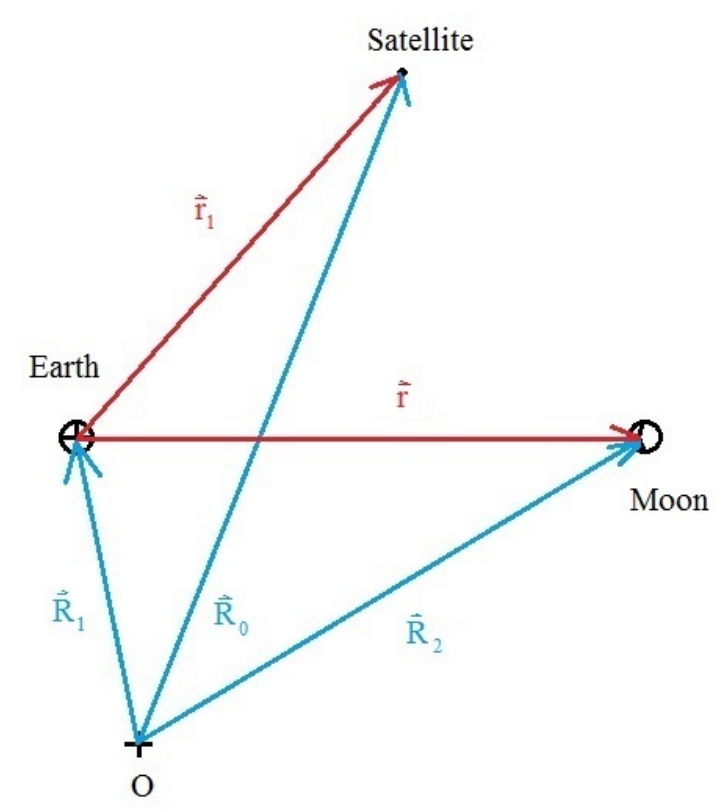

Figure 13. The position vectors for the Earth, Moon, and satellite with respect to an inertial origin are $\vec{R}_{1}, \vec{R}_{2}$, and $\vec{R}_{0}$. The position vectors for the Moon and satellite relative to the Earth are $\vec{r}$ and $\vec{r}_{1}$.

We shall begin our analysis with the fact that our equation of motion for the satellite is based upon the gravitational interaction between the satellite and both 
the Earth and the Moon (recalling that we have scaled the gravitational constant $G$ to unity). That is,

$$
\begin{gathered}
m_{0} \ddot{\overrightarrow{R_{0}}}=-m_{0} m_{2} \frac{\left(\vec{R}_{0}-\vec{R}_{2}\right)}{\left|\vec{R}_{0}-\vec{R}_{2}\right|^{3}}-m_{0} m_{1} \frac{\left(\vec{R}_{0}-\vec{R}_{1}\right)}{\left|\vec{R}_{0}-\vec{R}_{1}\right|^{3}}, \\
m_{0} \ddot{\overrightarrow{R_{0}}}=-m_{0} m_{2} \frac{\left(\vec{r}_{1}-\vec{r}\right)}{\left|\vec{r}_{1}-\vec{r}\right|^{3}}-m_{0} m_{1} \frac{\vec{r}_{1}}{\left|\vec{r}_{1}\right|^{3}} .
\end{gathered}
$$

The acceleration of the satellite relative to the Earth can be defined in terms of the inertial accelerations of the Earth and the satellite.

$$
\ddot{\vec{r}_{1}}=\ddot{\vec{R}}_{0}-\ddot{\vec{R}}_{1}
$$

Similarly, considering the equation of motion for the Earth,

$$
m_{1} \ddot{\vec{R}}_{1}=m_{1} m_{0} \frac{\vec{r}_{1}}{r_{1}^{3}}+m_{1} m_{2} \frac{\vec{r}}{r^{3}} .
$$

Dividing Equations 5.1 and 5.2 by $m_{0}$ and $m_{1}$ respectively, we obtain expressions for the inertial accelerations of the satellite and the Earth.

$$
\begin{gathered}
\ddot{\vec{R}}_{0}=-m_{2} \frac{\left(\vec{r}_{1}-\vec{r}\right)}{\left|r_{1}-r\right|^{3}}-m_{1} \frac{\vec{r}_{1}}{r_{1}^{3}}, \\
\ddot{\vec{R}}_{1}=\left[m_{0} \frac{\vec{r}_{1}}{r_{1}^{3}}+m_{2} \frac{\vec{r}}{r^{3}}\right] .
\end{gathered}
$$

Subtracting Equation 5.4 from Equation 5.3 yields the acceleration of the satellite with respect to the Earth, and it takes the following form:

$$
\ddot{\vec{r}}_{1}=\left[-m_{2} \frac{\left(\vec{r}_{1}-\vec{r}\right)}{\left|r_{1}-r\right|^{3}}-m_{1} \frac{\vec{r}_{1}}{r_{1}^{3}}-m_{0} \frac{\vec{r}_{1}}{r_{1}^{3}}-m_{2} \frac{\vec{r}}{r^{3}}\right] .
$$

We will show that this acceleration is the gradient of the sum of two functions.

$$
\ddot{\overrightarrow{r_{1}}}=\nabla_{0}\left(U_{0}+Q_{0}\right)
$$

where $\nabla_{0}$ refers to derivatives with respect to the position of the satellite. 
The first term $\left(U_{0}\right)$ is the potential energy per unit mass associated with the gravitational attraction between the Earth and the satellite (see Section 1.3).

$$
U_{0}=\frac{m_{1}+m_{0}}{r_{1}} .
$$

Taking the gradient,

$$
\nabla_{0} U_{0}=\nabla_{0}\left[\frac{m_{1}}{r_{1}}+\frac{m_{0}}{r_{1}}\right]=-\left[m_{1} \frac{\vec{r}_{1}}{r_{1}^{3}}+m_{0} \frac{\vec{r}_{1}}{r_{1}^{3}}\right] .
$$

We can see that these are the second and third terms of the acceleration of the satellite (Equation 5.5). Let us now assume that the other function $Q_{0}$ has the form

$$
Q_{0}=\left[-\frac{m_{2}}{\left|\vec{r}_{1}-\vec{r}\right|}-m_{2} \frac{\left(\vec{r}_{1} \cdot \vec{r}\right)}{r^{3}}\right] .
$$

This is the disturbing function. We will now show that taking the gradient of $Q_{0}$ gives us the first and fourth terms of the relative acceleration of the satellite with respect to the Earth (Equation 5.5).

$$
\begin{gathered}
\nabla_{0} Q_{0}=\nabla_{0}\left[-\frac{m_{2}}{\left|\overrightarrow{r_{1}}-\vec{r}\right|}-m_{2} \frac{\left(\vec{r}_{1} \cdot \vec{r}\right)}{r^{3}}\right], \\
\nabla_{0} Q_{0}=m_{2}\left[-\frac{\left(\overrightarrow{r_{1}}-\vec{r}\right)}{\left|\overrightarrow{r_{1}}-\vec{r}\right|^{3}}-\frac{\vec{r}}{r^{3}}\right] .
\end{gathered}
$$

We can see that the right hand side contains the first and fourth terms of the relative acceleration of the satellite (Equation 5.5). Therefore, the disturbing function is given by Equation 5.6. 


\section{CHAPTER 6}

\section{THE MASS LIMIT FOR STABILITY AT THE LAGRANGE POINTS}

An approach to finding the mass limit for stability at the Lagrange points is to find the acceleration of the third body relative to the center of mass of the other two bodies in the three-body system. Let the center of mass for the Earth and Moon be $C$. Let us call the position vector of the satellite relative to the center of mass of the other two bodies $\vec{\rho}$. See Figure 14 .

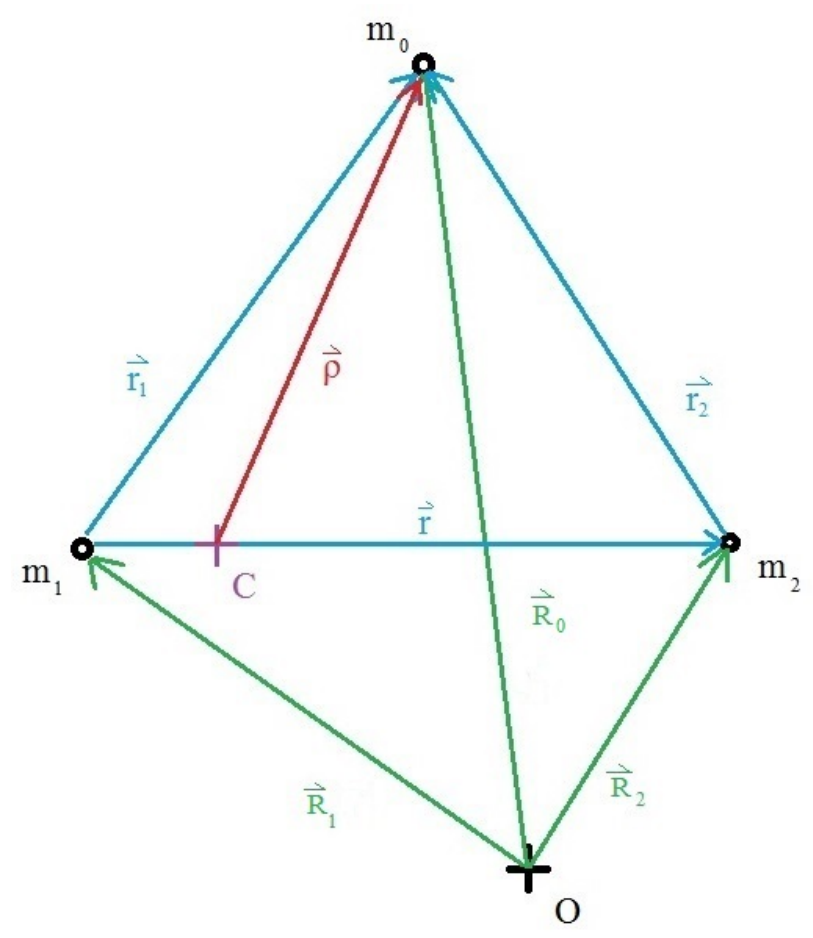

Figure 14. Positions of the three massive bodies relative to the inertial origin $(O)$. The position of the satellite with respect to the center of mass $(C)$ of the Earth and Moon is denoted by $\vec{\rho}$. See Figure 5 and discussion in Chapter 3 for remaining notation.

Let

$$
M=m_{1}+m_{2}+m_{0}
$$


and

$$
\sigma=m_{1}+m_{2}
$$

We begin with the equation of motion for $m_{1}$ in the inertial frame, recalling that the constant $G$ has been scaled to unity,

$$
m_{1} \ddot{\vec{R}}_{1}=m_{1} m_{2} \frac{\vec{r}}{r^{3}}+m_{1} m_{0} \frac{\vec{r}_{1}}{r_{1}^{3}}
$$

Dividing by $m_{1}$,

$$
\ddot{\vec{R}}_{1}=m_{2} \frac{\vec{r}}{r^{3}}+m_{0} \frac{\vec{r}_{1}}{r_{1}^{3}}
$$

but

$$
\vec{R}_{1}=\vec{R}_{2}-\vec{r}
$$

Therefore,

$$
\begin{aligned}
& \left(\ddot{\vec{R}}{ }_{2}-\ddot{\vec{r}}\right)=m_{2} \frac{\vec{r}}{r^{3}}+m_{0} \frac{\vec{r}_{1}}{r_{1}^{3}}, \\
& \ddot{\vec{r}}=\ddot{\vec{R}}_{2}-m_{0} \frac{\vec{r}_{1}}{r_{1}^{3}}-m_{2} \frac{\vec{r}}{r^{3}} .
\end{aligned}
$$

It can be shown that

$$
\begin{gathered}
\ddot{\vec{r}}_{2}=\ddot{\vec{R}}_{0}+m_{1} \frac{\vec{r}}{r^{3}}-m_{0} \frac{\overrightarrow{r_{2}}}{r_{2}^{3}}, \\
\ddot{\vec{r}}_{1}=-\ddot{\vec{R}}_{1}-m_{2} \frac{\overrightarrow{r_{2}}}{r_{2}^{3}}-m_{1} \frac{\overrightarrow{r_{1}}}{r_{1}^{3}} .
\end{gathered}
$$

Similarly for the other position vectors with respect to the origin, we can show that

$$
\begin{aligned}
& \ddot{\vec{R}}_{2}=-m_{1} \frac{\vec{r}}{r^{3}}+m_{0} \frac{\vec{r}_{2}}{r_{2}^{3}}, \\
& \ddot{\vec{R}}_{0}=-m_{1} \frac{\vec{r}_{1}}{r_{1}^{3}}-m_{2} \frac{\vec{r}_{2}}{r_{2}^{3}} .
\end{aligned}
$$


Now

$$
\begin{gathered}
\ddot{\vec{r}}=\ddot{\vec{R}}_{2}-\ddot{\vec{R}}_{1}, \\
\ddot{\vec{r}}=-m_{1} \frac{\vec{r}}{r^{3}}+m_{0} \frac{\vec{r}_{2}}{r_{2}^{3}}-m_{2} \frac{\vec{r}}{r^{3}}-m_{0} \frac{\vec{r}_{1}}{r_{1}^{3}}, \\
\ddot{\vec{r}}=-\sigma \frac{\vec{r}}{r^{3}}+m_{0}\left(\frac{\vec{r}_{2}}{r_{2}^{3}}-\frac{\vec{r}_{1}}{r_{1}^{3}}\right) .
\end{gathered}
$$

Also,

$$
\begin{gathered}
\ddot{\vec{r}}_{2}=\ddot{\vec{R}}_{0}-\ddot{\vec{R}}_{2}, \\
\ddot{\vec{r}}_{2}=-m_{1} \frac{\vec{r}_{1}}{r_{1}^{3}}-m_{2} \frac{\vec{r}_{2}}{r_{2}^{3}}+m_{1} \frac{\vec{r}}{r^{3}}-m_{0} \frac{\vec{r}_{2}}{r_{2}^{3}}, \\
\ddot{\vec{r}}_{2}=m_{1}\left(\frac{\vec{r}}{r^{3}}-\frac{\vec{r}_{1}}{r_{1}^{3}}\right)-\left(m_{2}+m_{0}\right) \frac{\vec{r}_{2}}{r_{2}^{3}} .
\end{gathered}
$$

To put this equation into a form that is more useful to us, let us consider the vector $\vec{\rho}$. From Section 2.4, the vector from $C$ to $m_{2}$ is $\frac{m_{1}}{m_{1+} m_{2}} \vec{r}=\frac{m_{1}}{\sigma} \vec{r}$. Thus $\vec{\rho}$ can be expressed as

$$
\vec{\rho}=\frac{m_{1}}{\sigma} \vec{r}+\vec{r}_{2}
$$

Thus, the acceleration vector of $m_{0}$ relative to $C$ is

$$
\ddot{\vec{\rho}}=\frac{m_{1}}{\sigma} \ddot{\vec{r}}+\ddot{\overrightarrow{r_{2}}} \text {. }
$$

Rearranging Equation 6.8,

$$
\overrightarrow{r_{2}}=\vec{\rho}-\frac{m_{1}}{\sigma} \vec{r}
$$

Since $\vec{r}_{1}$ can be written as

$$
\begin{gathered}
\vec{r}_{1}=\vec{r}+\vec{r}_{2}, \\
\vec{r}_{1}=\vec{r}+\vec{\rho}-\frac{m_{1}}{\sigma} \vec{r}=\vec{\rho}+\left(1-\frac{m_{1}}{\sigma}\right) \vec{r}=\vec{\rho}+\frac{m_{2}}{\sigma} \vec{r} .
\end{gathered}
$$


Substituting Equations 6.10 and 6.12 into Equation 6.6,

$$
\ddot{\vec{r}}=-\sigma \frac{\vec{r}}{r^{3}}+m_{0}\left(\frac{\vec{\rho}-\frac{m_{1}}{\sigma} \vec{r}}{\left|\vec{\rho}-\frac{m_{1}}{\sigma} \vec{r}\right|^{3}}-\frac{\vec{\rho}+\frac{m_{2}}{\sigma} \vec{r}}{\left|\vec{\rho}+\frac{m_{2}}{\sigma} \vec{r}\right|^{3}}\right) .
$$

Thus Equation 6.7 takes on the following form

$$
\ddot{\vec{r}}_{2}=m_{1}\left(\frac{\vec{r}}{r^{3}}-\frac{\vec{\rho}+\frac{m_{2}}{\sigma} \vec{r}}{\left|\vec{\rho}+\frac{m_{2}}{\sigma} \vec{r}\right|^{3}}\right)-\left(m_{2}+m_{0}\right)\left(\frac{\vec{\rho}-\frac{m_{1}}{\sigma} \vec{r}}{\left|\vec{\rho}-\frac{m_{1}}{\sigma} \vec{r}\right|^{3}}\right) .
$$

Substituting Equations 6.13 and 6.14 into Equation 6.9,

$$
\begin{gathered}
\ddot{\vec{\rho}}=\frac{m_{1}}{\sigma}\left[-\sigma \frac{\vec{r}}{r^{3}}+m_{0}\left(\frac{\vec{\rho}-\frac{m_{1}}{\sigma} \vec{r}}{\left|\vec{\rho}-\frac{m_{1}}{\sigma} \vec{r}\right|^{3}}-\frac{\vec{\rho}+\frac{m_{2}}{\sigma} \vec{r}}{\left|\vec{\rho}+\frac{m_{2}}{\sigma} r\right|^{3}}\right)\right] \\
+m_{1}\left(\frac{\vec{r}}{r^{3}}-\frac{\vec{\rho}+\frac{m_{2}}{\sigma} \vec{r}}{\left|\vec{\rho}+\frac{m_{2}}{\sigma} r\right|^{3}}\right)-\left(m_{2}+m_{0}\right)\left(\frac{\vec{\rho}-\frac{m_{1}}{\sigma} \vec{r}}{\left|\vec{\rho}-\frac{m_{1}}{\sigma} \vec{r}\right|^{3}}\right) .
\end{gathered}
$$

Grouping like terms,

$$
\begin{gathered}
\ddot{\vec{\rho}}=-m_{1} \frac{\vec{r}}{r^{3}}+m_{1} \frac{\vec{r}}{r^{3}}+\left(\frac{m_{1} m_{0}}{\sigma}+m_{1}\right)\left(-\frac{\vec{\rho}+\frac{m_{2}}{\sigma} \vec{r}}{\left|\vec{\rho}+\frac{m_{2}}{\sigma} \vec{r}\right|^{3}}\right) \\
+\left[\frac{m_{1} m_{0}}{\sigma}-\left(m_{2}+m_{0}\right)\right]\left(\frac{\vec{\rho}-\frac{m_{1}}{\sigma} \vec{r}}{\left|\vec{\rho}-\frac{m_{1}}{\sigma} \vec{r}\right|^{3}}\right) .
\end{gathered}
$$

Finding common denominators, cancelling the first two terms, and using $\sigma=m_{1}+m_{2}$ we obtain

$$
\begin{gathered}
\ddot{\vec{\rho}}=\left(\frac{m_{1} m_{0}}{\sigma}+\frac{m_{1}^{2}+m_{1} m_{2}}{\sigma}\right)\left(-\frac{\vec{\rho}+\frac{m_{2}}{\sigma} \vec{r}}{\left|\vec{\rho}+\frac{m_{2}}{\sigma} r\right|^{3}}\right) \\
+\left[\frac{m_{1} m_{0}}{\sigma}-\frac{\left(m_{1} m_{2}+m_{2}^{2}+m_{1} m_{0}+m_{2} m_{0}\right)}{\sigma}\right]\left(\frac{\vec{\rho}-\frac{m_{1}}{\sigma} \vec{r}}{\left|\vec{\rho}-\frac{m_{1}}{\sigma} \vec{r}\right|^{3}}\right) .
\end{gathered}
$$

Consequently,

$$
\ddot{\vec{\rho}}=-\frac{M}{\sigma}\left(m_{1} \frac{\vec{\rho}+\frac{m_{2}}{\sigma} \vec{r}}{\left|\vec{\rho}+\frac{m_{2}}{\sigma} r\right|^{3}}+m_{2} \frac{\vec{\rho}-\frac{m_{1}}{\sigma} \vec{r}}{\left|\vec{\rho}-\frac{m_{1}}{\sigma} \vec{r}\right|^{3}}\right) .
$$

Now we have an equation of motion for the satellite with respect to the center of mass of the primary and secondary masses. Note that since the mass of the 
satellite only appears in the $\frac{M}{\sigma}$ term, we can evaluate the effect of the mass of the satellite on its acceleration. If the acceleration of a massless satellite is $\ddot{\overrightarrow{\rho_{0}}}$, then the acceleration of a satellite with finite mass $m_{0}$ is

$$
\ddot{\vec{\rho}}=\left(1+\frac{m_{0}}{\sigma}\right) \ddot{\vec{\rho}} \text {. }
$$

Equation 6.16 is quite complicated; it must be solved numerically in order to determine the stability of a mass at this point. However, in the spirit of this thesis we are trying to discover an analytical solution and leave the numerical analysis of equation 6.16 for future exploration.

\subsection{The Net Effect at the $1 \%$ Threshold}

It is interesting to consider what effect increasing the mass of the system by $1 \%$ will have on the semi-major axis of the system's rotation and apply this to our three-body problem. According to Equation 1.5,

$$
\ddot{\vec{r}}+\sigma \frac{\vec{r}}{r^{3}}=0
$$

This describes the motion of two bodies orbiting a common center of mass in elliptical paths.

We also know that the equation of relative motion between the primary and secondary masses in a three-body system is described by the following equation of motion:

$$
\ddot{\vec{r}}+\sigma \frac{\vec{r}}{r^{3}}=\nabla Q_{0}
$$

where $Q_{0}$ is the disturbing function for the third body. We have evaluated this last term (see Equation 6.6), and our new equation of motion is

$$
\ddot{\vec{r}}+\sigma \frac{\vec{r}}{r^{3}}=m_{0}\left(\frac{\overrightarrow{r_{2}}}{r_{2}^{3}}-\frac{\vec{r}_{1}}{r_{1}^{3}}\right)
$$


Subtracting the second term on the left hand side of Equation 6.17 from both sides of the equation, we can rewrite our equation of motion in the following form:

$$
\ddot{\vec{r}}=-\sigma \frac{\vec{r}}{r^{3}}+m_{0}\left(\frac{\vec{r}_{2}}{r^{3}}-\frac{\vec{r}_{1}}{r_{1}^{3}}\right) .
$$

This equation represents the relative acceleration of the secondary mass with respect to the primary mass. It is clear that the second term on the right hand side is due to the third mass.

Let us assume that the satellite is at one of the triangular Lagrange points. Since this body is at the vertex of an equilateral triangle, we can see that the components of $-\vec{r}_{1}$ and $\vec{r}_{2}$ that are perpendicular to $\vec{r}$ are equal in magnitude and opposite in direction (see Figure 14). We can also see that the components of $-\vec{r}_{1}$ and $\vec{r}_{2}$ that are parallel to $\vec{r}$ are in the same direction (parallel to each other, not anti-parallel). Therefore, the second term on the right hand side of Equation 6.18 is directed toward $-\vec{r}$. This acceleration is equivalent to increasing the mass of either one or both of the primary and/or secondary masses since increasing this relative acceleration is equivalent to increasing the gravitational force.

It remains for us to discover how massive $m_{0}$ must be to change the net gravitational effect. To determine the effect of increasing the overall gravitational attraction between the primary and secondary mass by $1 \%$, we have the following expression:

$$
\text { (0.01) } \sigma \frac{\vec{r}}{r^{3}}=m_{0}\left(\frac{\vec{r}_{2}}{r_{2}^{3}}-\frac{\vec{r}_{1}}{r_{1}^{3}}\right) .
$$

Dividing by the parenthetical term on the right hand side,

$$
\frac{(0.01) \sigma \frac{\vec{r}}{r^{3}}}{\left(\frac{\vec{r}_{2}}{r_{2}^{3}}-\frac{\vec{r}_{1}}{r_{1}^{3}}\right)}=m_{0} .
$$


As before, all of the relative position vectors are equal in magnitude. Let

$$
\left|\frac{\vec{r}}{r^{3}}\right|=\left|\frac{\vec{r}_{2}}{r_{2}^{3}}\right|=\left|\frac{\vec{r}_{1}}{r_{1}^{3}}\right|=\alpha .
$$

Thus, our expression becomes

$$
\begin{gathered}
\frac{(0.01) \sigma \alpha}{2 \alpha}=m_{0}, \\
m_{0}=\frac{1}{2}(0.01) \sigma=\frac{1}{2}(0.01)\left(m_{1}+m_{2}\right) .
\end{gathered}
$$

Recall that $\sigma$ is the combined mass of the Earth and Moon. Now since $m_{0}$ is one half of one percent of these combined masses, this puts $m_{0}$ at just below half of the mass of the moon. Therefore, since

$$
m_{1}=5.972 \times 10^{24} \mathrm{~kg},
$$

and

$$
m_{2}=7.348 \times 10^{22} \mathrm{~kg},
$$

we conclude that

$$
m_{0} \leq 3.023 \times 10^{22} \mathrm{~kg} .
$$

We now consider how much such a satellite will cause the semi-major axis of the Earth-Moon system to vary. At apogee the Moon is $4.055 \times 10^{5} \mathrm{~km}$ from the Earth and $3.633 \times 10^{5} \mathrm{~km}$ at perigee (Roy, 2005). This is about $10 \%$ of the semi-major axis (or $\pm 5 \%$ from the mean).

The semi-major axis of a two-body system is related to the masses and the total energy as follows:

$$
a=-\frac{G m_{1} m_{2}}{2 E} .
$$

The net change in this semi-major axis is due to the increased mass in the system, which we stated to be equivalent to increasing the mass of one or the other 
(or both) massive bodies. To increase the overall gravitational attraction between the primary and secondary masses by one percent, then one must increase the product of their masses by one percent. The numerator and the potential energy term in Equation 6.19 both contain this mass product. According to the Virial Theorem,

$$
-U=2 T .
$$

Increasing the mass of the system increases the kinetic energy and the potential energy. Note that the potential energy is negative since it is the potential energy of a gravitationally bound system. Thus the numerator in Equation 6.19 will increase by $1 \%$. The initial total energy is

$$
\begin{gathered}
E=U+T=-2 T+T=-T, \\
2 E=-2 T .
\end{gathered}
$$

Since the potential energy and kinetic energy both increase by one percent, the new total energy $\left(E^{\prime}\right)$ is

$$
E^{\prime}=(1.01) U+(1.01) T
$$

The denominator in Equation 6.19 is twice the total energy

$$
2 E^{\prime}=2[(1.01) U+(1.01) T]=(2.02)(-2 T)+(2.02) T=-4.04 T+2.02 T .
$$

Thus, the denominator term in Equation 6.19 is

$$
2 E^{\prime}=-2.02 T \text {. }
$$

The numerator and denominator both increase in total by $1 \%$. Thus, the semi-major axis remains unchanged. This is not absolutely correct, since the kinetic energy depends upon the sum of the masses and not on the product of the masses as 
is the potential energy. Treating this as a limiting case, an increase in kinetic energy of $1 \%$ does not change the semi-major axis of the system.

Let us take the opposite limiting case where the change in kinetic energy is zero. In this case, only the potential energy increases by $1 \%$, and the total energy becomes

$$
E^{\prime}=(1.01) U+T .
$$

The denominator of Equation 6.19 is still twice the total energy.

$2 E^{\prime}=2[(1.01) U+T]=(2.02) U+2 T=(2.02)(-2 T)+2 T=-4.04 T+2 T$.

Thus, the denominator of Equation 6.19 becomes

$$
2 E^{\prime}=-2.04 T \text {. }
$$

The numerator of Equation 6.19 increases by one percent, and the denominator increases by four percent. Thus, the semi-major axis will contract to just over $97 \%$ of its original length. It should be noted that this is only a theoretical case where the kinetic energy does not increase. The actual increase in energy is much closer to the first limiting case.

\subsection{Gascheau's Stability Condition}

As we have shown the equilateral triangle configuration for mutually gravitationally attractive bodies is stable for two finite masses and an infinitesimal mass. Furthermore, as long as these masses are circularly orbiting a common center of mass, the configuration is stable as long as the masses obey the critical mass ratio. Gascheau (1843) was able to show that this configuration is stable if

$$
\frac{\left(m_{1}+m_{2}+m_{0}\right)^{2}}{m_{1} \cdot m_{2}+m_{1} \cdot m_{0}+m_{2} \cdot m_{0}}>27 .
$$


E. J. Routh (1875) is largely accredited with the discovery of the critical mass ratio, but Gascheau pubished this result 32 years earlier. We obtained a copy of Gascheau's work, and it is described in the Appendix.

Routh was also able to show that there are stability limits with regards to the attractive force between the bodies. If the force varies as the inverse $n^{\text {th }}$ power of the distance, the system cannot be stable if $n$ is greater than 2. If $n$ is less than -1 the system is always stable. For $n$ between -1 and 2, the following condition gives a stable configuration:

$$
\frac{\left(m_{1}+m_{2}+m_{0}\right)^{2}}{m_{1} \cdot m_{2}+m_{1} \cdot m_{0}+m_{2} \cdot m_{0}}>3\left(\frac{1+n}{3-n}\right)^{2} .
$$

Since the force we are concerned with is gravitational in nature, $n=2$. Thus, Routh's expression (Equation 6.21) reduces to Gascheau's (Equation 6.20). If we insert the values for $m_{1}, m_{2}$, and $m_{0}$ from Section 6.1 into Equation 6.20, we can see readily that this configuration is stable. That is

$$
\begin{gathered}
\frac{\left(m_{1}+m_{2}+m_{0}\right)^{2}}{m_{1} \cdot m_{2}+m_{1} \cdot m_{0}+m_{2} \cdot m_{0}}>27, \\
\left(m_{1}+m_{2}+m_{0}\right)^{2}>27\left(m_{1} \cdot m_{2}+m_{1} \cdot m_{0}+m_{2} \cdot m_{0}\right), \\
3.692 \times 10^{49} \mathrm{~kg}^{2}>1.678 \times 10^{49} \mathrm{~kg}^{2} .
\end{gathered}
$$

According to this relation, the actual mass limit for the satellite $\left(m_{0}\right)$ is more than double the mass of the moon.

$$
m_{0}<1.636 \times 10^{23} \mathrm{~kg} .
$$

This is an advantageous result for placing asteroids at the triangular Lagrange points since none of the near Earth asteroids are a significant fraction of the Moon's mass. The largest known near Earth asteroid (433 Eros) has a mass of about $7.2 \times 10^{15} \mathrm{~kg}$. Thus, according to Gascheau's result the system should be stable. 
In conclusion, in this chapter we have derived an expression for the acceleration of the satellite with respect to the center of mass of the primary and secondary. This acceleration depends on the mass of the satellite and is consequently the main result we set out to determine. Instead of carrying out a numerical determination of the maximum mass of the satellite for stability we opted to consider an energy analysis and showed the effect of a massive satellite on the semi-major axis of the Earth-Moon system. We also stated the results of Gascheau (1843) and Routh (1875) who evaluated the upper limit of the satellite mass for stability. 


\section{CHAPTER 7}

\section{A SPECIAL CASE OF THE FOUR BODY PROBLEM}

In this chapter, we consider placing masses at both of the triangular Lagrange points. If these masses are equal in magnitude, it will be shown that the net gravitational force on the secondary body (the Moon) is directed toward the primary mass (the Earth). We shall determine how much the semi-major axis of the system changes using the technique of Section 6.1.

As illustrated in Figure 15, the $y$-components of the gravitational forces on the Moon from masses 1 and 2 are anti-parallel. Since we are supposing that these two

masses are equal in magnitude, we can argue by symmetry that $\vec{F}_{1_{y}}+\vec{F}_{2_{y}}=0$. Thus, the only remaining components of the gravitational forces on the Moon from the two added masses are both directed toward the Earth. Therefore, the net force on the Moon is directed toward the Earth.

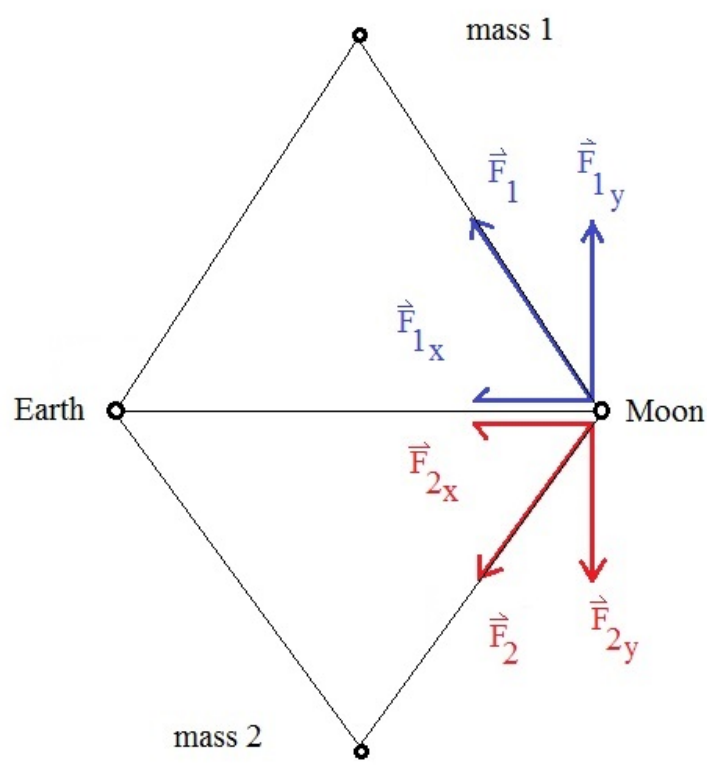

Figure 15. Gravitational forces of masses 1 and 2 on the Moon and their orthogonal components. Note that $\vec{F}_{1_{y}}$ and $\vec{F}_{2_{y}}$ are equal in magnitude and opposite in direction, and $\vec{F}_{1_{x}}$ and $\vec{F}_{2_{x}}$ are directed toward the Earth. 
To derive the disturbing function for our system in the simplest possible manner, we will consider a sytem with three bodies. We will develop a proof in general so we can add more bodies at a later time using the same notation. Let the central mass be denoted by $M$, and let the other two masses be $m_{i}$ and $m_{j}$. Let $\xi_{i}$ and $\eta_{i}$ be the coordinates of the $i^{\text {th }}$ body relative to an inertial origin, and let $\xi_{0}$ and $\eta_{0}$ be the coordinates of the primary mass relative to the same origin. Also let $r_{i}$ be the position of mass $i$ with respect to the central mass, $r_{j}$ be the position of mass $j$ with respect to the central mass, and $r_{i j}$ be the position of mass $j$ with respect to mass $i$. Also let $\gamma_{i}$ and $\delta_{i}$ be the relative coordinates between mass $i$ and the central mass. They are defined by

$$
\gamma_{i}=\xi_{i}-\xi_{0}
$$

and

$$
\delta_{i}=\eta_{i}-\eta_{0}
$$

Therefore,

$$
r_{i}=\left[\left(\xi_{i}-\xi_{0}\right)^{2}+\left(\eta_{i}-\eta_{0}\right)^{2}\right]^{\frac{1}{2}}=\left[\gamma_{i}^{2}+\delta_{i}^{2}\right]^{\frac{1}{2}} .
$$

Let us define the force function $V$ as the negative of the potential energy. That is,

$$
V=\frac{M m_{j}}{r_{j}}+\frac{M m_{i}}{r_{i}}+\frac{m_{i} m_{j}}{r_{i j}} .
$$

Newton's second law is

$$
F_{i_{\xi}}=m_{i} a_{i_{\xi}}
$$

However,

$$
F_{i_{\xi}}=\frac{\partial V}{\partial \xi_{i}} .
$$


Since the $\xi$-component of the acceleration of body $i$ is $\frac{d^{2} \xi_{i}}{d t^{2}}$,

$$
a_{i_{\xi}}=\frac{d^{2} \xi_{i}}{d t^{2}}
$$

but

$$
\begin{gathered}
\frac{\partial V}{\partial \xi_{i}}=m_{i} \frac{d^{2} \xi_{i}}{d t^{2}} \\
\frac{\partial V}{\partial \xi_{i}}=\frac{\partial}{\partial \xi_{i}}\left[\frac{M m_{i}}{\left[\left(\xi_{i}-\xi_{0}\right)^{2}+\left(\eta_{i}-\eta_{0}\right)^{2}\right]^{\frac{1}{2}}}+\frac{m_{i} m_{j}}{\left[\left(\xi_{i}-\xi_{j}\right)^{2}+\left(\eta_{i}-\eta_{j}\right)^{2}\right]^{\frac{1}{2}}}\right], \\
\frac{\partial V}{\partial \xi_{i}}=-m_{i}\left[\frac{M\left(\xi_{i}-\xi_{0}\right)}{r_{i}^{3}}+\frac{m_{j}\left(\xi_{i}-\xi_{j}\right)}{r_{i j}^{3}}\right]
\end{gathered}
$$

therefore,

$$
\frac{d^{2} \xi_{i}}{d t^{2}}=-\left[\frac{M\left(\xi_{i}-\xi_{0}\right)}{r_{i}^{3}}+\frac{m_{j}\left(\xi_{i}-\xi_{j}\right)}{r_{i j}^{3}}\right]
$$

Similarly,

$$
\begin{gathered}
F_{0_{\xi}}=\frac{\partial V}{\partial \xi_{0}}, \\
\frac{\partial V}{\partial \xi_{0}}=\frac{\partial}{\partial \xi_{0}}\left[\frac{M m_{i}}{\left[\left(\xi_{i}-\xi_{0}\right)^{2}+\left(\eta_{i}-\eta_{0}\right)^{2}\right]^{\frac{1}{2}}}+\frac{M m_{j}}{\left[\left(\xi_{j}-\xi_{0}\right)^{2}+\left(\eta_{j}-\eta_{0}\right)^{2}\right]^{\frac{1}{2}}}\right], \\
\frac{\partial V}{\partial \xi_{0}}=M\left[\frac{m_{i}\left(\xi_{i}-\xi_{0}\right)}{r_{i}^{3}}+\frac{m_{j}\left(\xi_{j}-\xi_{0}\right)}{r_{j}^{3}}\right],
\end{gathered}
$$

therefore,

$$
\frac{d^{2} \xi_{0}}{d t^{2}}=\left[\frac{m_{i}\left(\xi_{i}-\xi_{0}\right)}{r_{i}^{3}}+\frac{m_{j}\left(\xi_{j}-\xi_{0}\right)}{r_{j}^{3}}\right] .
$$

The second derivative of $\gamma_{i}$ with respect to time is

$$
\frac{d^{2} \gamma_{i}}{d t^{2}}=\frac{d^{2} \xi_{i}}{d t^{2}}-\frac{d^{2} \xi_{0}}{d t^{2}}
$$


Inserting Equations 7.5 and 7.6 into Equation 7.7,

$$
\frac{d^{2} \gamma_{i}}{d t^{2}}=-\frac{M\left(\xi_{i}-\xi_{0}\right)}{r_{i}^{3}}-\frac{m_{j}\left(\xi_{i}-\xi_{j}\right)}{r_{i j}^{3}}-\frac{m_{i}\left(\xi_{i}-\xi_{0}\right)}{r_{i}^{3}}-\frac{m_{j}\left(\xi_{j}-\xi_{0}\right)}{r_{j}^{3}} .
$$

The first two terms of Equation 7.8 can be written as

$$
-\frac{M\left(\xi_{i}-\xi_{0}\right)}{r_{i}^{3}}-\frac{m_{j}\left(\xi_{i}-\xi_{j}\right)}{r_{i j}^{3}}=\frac{\partial}{\partial \xi_{i}}\left(\frac{M}{r_{i}}+\frac{m_{j}}{r_{i j}}\right) .
$$

Therefore, Equation 7.8 can be written as

$$
\frac{d^{2} \gamma_{i}}{d t^{2}}=\frac{\partial}{\partial \xi_{i}}\left(\frac{M}{r_{i}}+\frac{m_{j}}{r_{i j}}\right)-\frac{m_{i}\left(\xi_{i}-\xi_{0}\right)}{r_{i}^{3}}-\frac{m_{j}\left(\xi_{j}-\xi_{0}\right)}{r_{j}^{3}} .
$$

The last two terms can be written as partial derivatives with respect to $\xi_{i}$

$$
-\frac{m_{i}\left(\xi_{i}-\xi_{0}\right)}{r_{i}^{3}}=\frac{\partial}{\partial \xi_{i}} \frac{m_{i}}{r_{i}}
$$

and

$$
-\frac{m_{j}\left(\xi_{j}-\xi_{0}\right)}{r_{j}^{3}}=-\frac{\partial}{\partial \xi_{i}} m_{j} \frac{\left(\xi_{i}-\xi_{0}\right)\left(\xi_{j}-\xi_{0}\right)+\left(\eta_{i}-\eta_{0}\right)\left(\eta_{j}-\eta_{0}\right)}{r_{j}^{3}} .
$$

Thus, Equation 7.8 can be written

$$
\begin{aligned}
\frac{d^{2} \gamma_{i}}{d t^{2}}=\frac{\partial}{\partial \xi_{i}}\left(\frac{M+m_{i}}{r_{i}}\right)+ & \frac{\partial}{\partial \xi_{i}}\left(\frac{m_{j}}{r_{i j}}-m_{j} \frac{\left(\xi_{i}-\xi_{0}\right)\left(\xi_{j}-\xi_{0}\right)+\left(\eta_{i}-\eta_{0}\right)\left(\eta_{j}-\eta_{0}\right)}{r_{j}^{3}}\right) \\
\frac{d^{2} \gamma_{i}}{d t^{2}} & =\frac{\partial}{\partial \xi_{i}}\left(\frac{M+m_{i}}{r_{i}}+Q_{i j}\right)
\end{aligned}
$$

where

$$
Q_{i j}=m_{j}\left(\frac{1}{r_{i j}}-\frac{\left(\xi_{i}-\xi_{0}\right)\left(\xi_{j}-\xi_{0}\right)+\left(\eta_{i}-\eta_{0}\right)\left(\eta_{j}-\eta_{0}\right)}{r_{j}^{3}}\right) .
$$

Now,

$$
\overrightarrow{r_{i}} \cdot \overrightarrow{r_{j}}=\left|r_{i}\right|\left|r_{j}\right| \cos \left(\theta_{i}-\theta_{j}\right)
$$


(see Figure 16) where

$$
\cos \left(\theta_{i}-\theta_{j}\right)=\frac{\overrightarrow{r_{i}} \cdot \overrightarrow{r_{j}}}{\left|r_{i}\right|\left|r_{j}\right|}=\frac{\left(\xi_{i}-\xi_{0}\right)\left(\xi_{j}-\xi_{0}\right)+\left(\eta_{i}-\eta_{0}\right)\left(\eta_{j}-\eta_{0}\right)}{\left|r_{i}\right|\left|r_{j}\right|} .
$$

Multipying by $\frac{r_{i}}{r_{j}^{2}}$ yields

$$
\frac{r_{i} \cos \left(\theta_{i}-\theta_{j}\right)}{r_{j}^{2}}=\frac{\left(\xi_{i}-\xi_{0}\right)\left(\xi_{j}-\xi_{0}\right)+\left(\eta_{i}-\eta_{0}\right)\left(\eta_{j}-\eta_{0}\right)}{r_{j}^{3}} .
$$

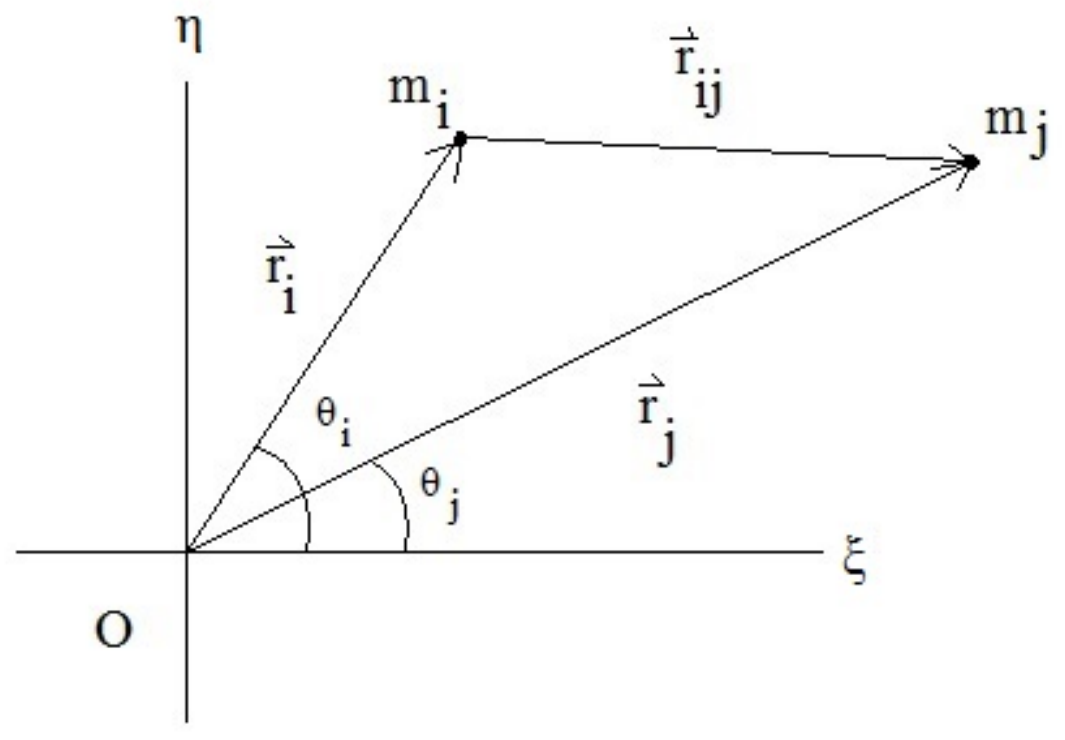

Figure 16. Relative positions of the $i^{t h}$ and $j^{\text {th }}$ masses with respect to the origin $(O)$ at the primary mass.

In polar coordinates Equation 7.11 can be written

$$
Q_{i j}=m_{j}\left[\frac{1}{r_{i j}}-\frac{r_{i}}{r_{j}^{2}} \cos \left(\theta_{i}-\theta_{j}\right)\right]
$$

where

$$
r_{i j}=\left[r_{i}^{2}+r_{j}^{2}-2 r_{i} r_{j} \cos \left(\theta_{i}-\theta_{j}\right)\right]^{\frac{1}{2}}
$$


Note that $Q_{i j}$ is a function of $r_{i}, r_{j}, \theta_{i}$, and $\theta_{j}$. Let us express $\overrightarrow{r_{i}}$ as

$$
\overrightarrow{r_{i}}=\gamma_{i} \hat{\xi}+\delta_{i} \hat{\eta}
$$

The second time derivative of $\overrightarrow{r_{i}}$ is

$$
\begin{gathered}
\frac{d^{2} \overrightarrow{r_{i}}}{d t^{2}}=\frac{d^{2} \gamma_{i}}{d t^{2}} \hat{\xi}+\frac{d^{2} \delta_{i}}{d t^{2}} \hat{\eta}, \\
\frac{d^{2} \overrightarrow{r_{i}}}{d t^{2}}=\left[\hat{\xi} \frac{\partial}{\partial \xi_{i}}+\hat{\eta} \frac{\partial}{\partial \eta_{i}}\right]\left(\frac{M+m_{i}}{r_{i}}+Q_{i j}\right) .
\end{gathered}
$$

The right hand side is the gradient of $\left(\frac{M+m_{i}}{r_{i}}+Q_{i j}\right)$.

$$
\frac{d^{2} \vec{r}_{i}}{d t^{2}}=\nabla_{i}\left(\frac{M+m_{i}}{r_{i}}+Q_{i j}\right) .
$$

The two dimensional gradient in polar coordinates is

$$
\nabla=\hat{r} \frac{\partial}{\partial r}+\hat{\theta} \frac{1}{r} \frac{\partial}{\partial \theta}
$$

The acceleration of a body in two dimensions in polar coordinates is

$$
\left(\ddot{r}-r \dot{\theta}^{2}\right) \hat{r}+(r \ddot{\theta}+2 \dot{r} \dot{\theta}) \hat{\theta} .
$$

Therefore,

$$
\left(\ddot{r}_{i}-r_{i} \dot{\theta}_{i}^{2}\right) \hat{r}+\left(r_{i} \ddot{\theta}_{i}+2 \dot{r}_{i} \dot{\theta}_{i}\right) \hat{\theta}=\left[\hat{r} \frac{\partial}{\partial r_{i}}+\hat{\theta} \frac{1}{r_{i}} \frac{\partial}{\partial \theta_{i}}\right]\left(\frac{M+m_{i}}{r_{i}}+Q_{i j}\right) .
$$

Examining the $\theta$-components of this equation,

$$
\left(r_{i} \ddot{\theta}_{i}+2 \dot{r}_{i} \dot{\theta}_{i}\right)=\frac{1}{r_{i}} \frac{\partial}{\partial \theta_{i}}\left(\frac{M+m_{i}}{r_{i}}+Q_{i j}\right) .
$$

Note that the $\frac{M+m_{i}}{r_{i}}$ term has no explicit $\theta_{i}$ dependence. Thus, our expresession becomes

$$
\left(r_{i} \ddot{\theta}_{i}+2 \dot{r}_{i} \dot{\theta}_{i}\right)=\frac{1}{r_{i}} \frac{\partial Q_{i j}}{\partial \theta_{i}},
$$


or,

$$
\left(r_{i}^{2} \ddot{\theta}_{i}+2 r_{i} \dot{r}_{i} \dot{\theta}_{i}\right)=\frac{\partial Q_{i j}}{\partial \theta_{i}}
$$

The left hand side can be written

$$
r_{i}^{2} \ddot{\theta}_{i}+2 r_{i} \dot{r}_{i} \dot{\theta}_{i}=\frac{d}{d t}\left(r_{i}^{2} \dot{\theta}_{i}\right)
$$

Therefore,

$$
\frac{d}{d t}\left(r_{i}^{2} \frac{d \theta_{i}}{d t}\right)=\frac{\partial Q_{i j}}{\partial \theta_{i}}
$$

Let us briefly examine Equation 7.16. The function $Q_{i j}$ is the disturbing function (see Equation 5.6) but expressed more generally. We will show that Equation 7.16 will lead us to an expression that shows that the relative angular separation of the bodies is affected by the disturbing function.

Let $n_{i}$ be the mean motion of the $i^{\text {th }}$ body, and let the semi-major axis of its orbit be $a_{i}$. Its orbit will be perturbed by gravitational interactions with the other bodies. Let the perturbations in mean motion and semi-major axis be $\delta n_{i}$ and $\delta a_{i}$ respectively. Thus,

$$
\begin{gathered}
\frac{d \theta_{i}}{d t}=n_{i}+\delta n_{i}, \\
a_{i}=\left(a_{i_{0}}+\delta a_{i}\right) .
\end{gathered}
$$

Kepler's third law is

$$
n^{2} a^{3}=\sigma
$$

therefore, taking the differential

$$
\begin{gathered}
2 n a^{3} \delta n+3 n^{2} a^{2} \delta a=0, \\
\delta a=-\frac{2}{3} \frac{a}{n} \delta n .
\end{gathered}
$$


Consequently,

$$
\delta a_{i}=-\frac{2}{3} \frac{a_{i_{0}}}{n_{i}} \delta n_{i}
$$

Therefore,

$$
a_{i}=a_{i_{0}}\left(1-\frac{2}{3} \frac{\delta n_{i}}{n_{i}}\right)
$$

We now assume circular orbits for which $r_{i}=a_{i}$, and Equation 7.16 becomes

$$
\frac{d}{d t}\left(a_{i}^{2} \frac{d \theta_{i}}{d t}\right)=\frac{\partial Q_{i j}}{\partial \theta_{i}}
$$

If we substitute Equations 7.17 and 7.19 into this equation we obtain the following expression:

$$
\frac{d}{d t}\left(\left(a_{i_{0}}\left(1-\frac{2}{3} \frac{\delta n_{i}}{n_{i}}\right)\right)^{2}\left(n_{i}+\delta n_{i}\right)\right)=\frac{\partial Q_{i j}}{\partial \theta_{i}}
$$

Denoting the first term in parentheses by $\Delta^{2}$,

$$
\Delta^{2}=\left(a_{i_{0}}-\frac{2 a_{i_{0}}}{3 n_{i}} \delta n_{i}\right)^{2}
$$

So,

$$
\Delta^{2}=a_{i_{0}}^{2}-\frac{4 a_{i_{0}}^{2}}{3 n_{i}} \delta n_{i}+\frac{4 a_{i_{0}}^{2}}{9 n_{i}^{2}} \delta n_{i}^{2} .
$$

Since the perturbations are small, let us ignore the second order term and write

$$
\Delta^{2} \doteq a_{i_{0}}^{2}-\frac{4 a_{i_{0}}^{2}}{3 n_{i}} \delta n_{i}
$$

Thus,

$$
\Delta^{2}\left(n_{i}+\delta n_{i}\right)=a_{i_{0}}^{2} n_{i}-\frac{4 a_{i_{0}}^{2}}{3 n_{i}} n_{i} \delta n_{i}+a_{i_{0}}^{2} \delta n_{i}-\frac{4 a_{i_{0}}}{3 n_{i}} \delta n_{i}^{2}
$$

Again ignoring the second order term in $\delta n_{i}$,

$$
\Delta^{2}\left(n_{i}+\delta n_{i}\right) \doteq a_{i_{0}}^{2} n_{i}-\frac{4 a_{i_{0}}^{2}}{3} \delta n_{i}+a_{i_{0}}^{2} \delta n_{i}
$$


Noting that $n_{i}+\delta n_{i}=\frac{d \theta_{i}}{d t}$, we appreciate that this is the equivalent to $a_{i}^{2} \frac{d \theta_{i}}{d t}$.

Consequently,

$$
\frac{d}{d t}\left(a_{i}^{2} \frac{d \theta_{i}}{d t}\right)=\frac{d}{d t}\left(\Delta^{2} \frac{d \theta_{i}}{d t}\right)
$$

So

$$
\frac{d}{d t}\left(a_{i}^{2} \frac{d \theta_{i}}{d t}\right)=-\frac{1}{3} a_{i_{0}}^{2} \frac{d\left(\delta n_{i}\right)}{d t}=\frac{\partial Q_{i j}}{\partial \theta_{i}} .
$$

Solving for the partial derivative on the right hand side (by Equation 7.12 and $7.13)$,

$$
\frac{\partial Q_{i j}}{\partial \theta_{i}}=m_{j} \frac{\partial}{\partial \theta_{i}}\left\{\left[r_{i}^{2}+r_{j}^{2}-2 r_{i} r_{j} \cos \left(\theta_{i}-\theta_{j}\right)\right]^{-\frac{1}{2}}-\frac{r_{i}}{r_{j}^{2}} \cos \left(\theta_{i}-\theta_{j}\right)\right\} .
$$

Evaluating the first term in $\frac{\partial Q_{i j}}{\partial \theta_{i}}$,

$$
\frac{\partial}{\partial \theta_{i}}\left[r_{i}^{2}+r_{j}^{2}-2 r_{i} r_{j} \cos \left(\theta_{i}-\theta_{j}\right)\right]^{-\frac{1}{2}}=\frac{\left(r_{i} r_{j}\right)}{r_{i j}^{3}}\left(-\sin \left(\theta_{i}-\theta_{j}\right)\right) \text {. }
$$

Evaluating the second term in $\frac{\partial Q_{i j}}{\partial \theta_{i}}$,

$$
\frac{\partial}{\partial \theta_{i}}\left[\frac{r_{i}}{r_{j}^{2}} \cos \left(\theta_{i}-\theta_{j}\right)\right]=-\frac{r_{i}}{r_{j}^{2}} \sin \left(\theta_{i}-\theta_{j}\right) .
$$

Thus,

$$
\begin{aligned}
\frac{\partial Q_{i j}}{\partial \theta_{i}}= & m_{j}\left\{-\frac{r_{i} r_{j}}{r_{i j}^{3}} \sin \left(\theta_{i}-\theta_{j}\right)+\frac{r_{i}}{r_{j}^{2}} \sin \left(\theta_{i}-\theta_{j}\right)\right\} \\
& =-m_{j} \sin \left(\theta_{i}-\theta_{j}\right)\left\{\frac{r_{i} r_{j}}{r_{i j}^{3}}-\frac{r_{i}}{r_{j}^{2}}\right\} .
\end{aligned}
$$

Substituting $a_{0}$ for $r_{i}$ and $r_{j}$ since the $i^{t h}$ and $j^{t h}$ masses are at the vertices of equilateral triangles, by Equation 7.13,

$$
r_{i j}=\left\{2 a_{0}^{2}-2 a_{0}^{2} \cos \left(\theta_{i}-\theta_{j}\right)\right\}^{\frac{1}{2}}=\left\{2 a_{0}^{2}\left[1-\cos \left(\theta_{i}-\theta_{j}\right)\right]\right\}^{\frac{1}{2}} .
$$


Let us invoke the following trigonometric identity:

$$
1-\cos \phi=2 \sin ^{2} \frac{\phi}{2}
$$

Thus it follows that:

$$
\begin{gathered}
r_{i j}=\left\{2 a_{0}^{2} 2 \sin ^{2} \frac{\left(\theta_{i}-\theta_{j}\right)}{2}\right\}^{\frac{1}{2}}=2 a_{0} \sin \frac{\left(\theta_{i}-\theta_{j}\right)}{2}, \\
r_{i j}^{-3}=\left\{8 a_{0}^{3}\left|\sin \frac{\left(\theta_{i}-\theta_{j}\right)}{2}\right|^{3}\right\}^{-1}, \\
\frac{\partial Q_{i j}}{\partial \theta_{i}}=-m_{j} \sin \left(\theta_{i}-\theta_{j}\right)\left\{\frac{a_{0}^{2}}{8 a_{0}^{3}\left|\sin \frac{\left(\theta_{i}-\theta_{j}\right)}{2}\right|^{3}}-\frac{1}{a_{0}}\right\}, \\
\frac{\partial Q_{i j}}{\partial \theta_{i}}=\frac{-m_{j} \sin \left(\theta_{i}-\theta_{j}\right)}{a_{0}}\left\{\frac{1}{8\left|\sin \frac{\left(\theta_{i}-\theta_{j}\right)}{2}\right|^{3}}-1\right\} .
\end{gathered}
$$

By Equations 7.20 and 7.22,

$$
\begin{aligned}
& -\frac{1}{3} a_{0}^{2} \frac{d\left(\delta n_{i}\right)}{d t}=\frac{-m_{j} \sin \left(\theta_{i}-\theta_{j}\right)}{a_{0}}\left\{\frac{1}{8\left|\sin \frac{\left(\theta_{i}-\theta_{j}\right)}{2}\right|^{3}}-1\right\}, \\
& -\frac{1}{3} \frac{d\left(\delta n_{i}\right)}{d t}=\frac{-m_{j} \sin \left(\theta_{i}-\theta_{j}\right)}{a_{0}^{3}}\left\{\frac{1}{8\left|\sin \frac{\left(\theta_{i}-\theta_{j}\right)}{2}\right|^{3}}-1\right\} .
\end{aligned}
$$

By Kepler's third law,

$$
\begin{gathered}
n_{0}^{2} a_{0}^{3}=\left(M+m_{i}\right), \\
a_{0}^{3}=\frac{\left(M+m_{i}\right)}{n_{0}^{2}},
\end{gathered}
$$

therefore,

$$
\frac{1}{3} \frac{d\left(\delta n_{i}\right)}{d t}=-n \frac{m_{j} \sin \left(\theta_{i}-\theta_{j}\right)}{\left(M+m_{i}\right)}\left\{\frac{1}{8\left|\sin \frac{\left(\theta_{i}-\theta_{j}\right)}{2}\right|^{3}}-1\right\}
$$


This equation shows how $m_{j}$ affects the mean motion of $m_{i}$. The mass of the Earth is at least nine orders of magnitude greater than any of the near Earth asteroids. Thus, let us make the following approximation:

$$
\left(M+m_{i}\right) \doteq M
$$

Reinserting into the right hand side of Equation 7.23,

$$
\frac{1}{3} \frac{d\left(\delta n_{i}\right)}{d t}=n_{0}^{2} \frac{m_{j} \sin \left(\theta_{i}-\theta_{j}\right)}{M}\left\{\frac{1}{8\left|\sin \frac{\left(\theta_{i}-\theta_{j}\right)}{2}\right|^{3}}-1\right\} .
$$

This is for the case of three bodies. However, if there are four bodies, then the mean motion of mass $m_{i}$ will be affected by not one but two $m_{j}$ 's. Thus, summing over the $m_{j}$ 's our equation becomes

$$
\frac{1}{3} \frac{d\left(\delta n_{i}\right)}{d t}=n_{0}^{2} \sum_{j} \frac{m_{j} \sin \left(\theta_{i}-\theta_{j}\right)}{M}\left\{\frac{1}{8\left|\sin \frac{\left(\theta_{i}-\theta_{j}\right)}{2}\right|^{3}}-1\right\} .
$$

Note that when $\sin \left(\theta_{i}-\theta_{j}\right)$ is precisely $60^{\circ}$, the right hand side goes to zero since the denominator of the first term in braces becomes unity and reduces the entire term in braces to zero. This means that the rate of change of $\delta n_{i}$ is zero if the angular separation between $m_{i}$ and $m_{j}$ is $60^{\circ}$. If $\left(\theta_{i}-\theta_{j}\right)$ is greater than $60^{\circ}$, then the time derivative of $\delta n_{i}$ will be negative; this means that $m_{i}$ will experience an angular acceleration that decreases the rate of change of $\delta n_{i}$. Conversely, if $\left(\theta_{i}-\theta_{j}\right)$ is less than $60^{\circ}$, then the time derivative of $\delta n_{i}$ will be positive; this means that $m_{i}$ will experience an angular acceleration that increases the rate of change of $\delta n_{i}$.

If the system is revolving about the central mass $M$ with mean motion $n_{0}$, and the angular separation between two bodies $m_{i}$ and $m_{j}$ is exactly $60^{\circ}$, then $\frac{d\left(\delta n_{i}\right)}{d t}$ will be zero. The angular separation between the bodies will not change. This result agrees with Lagrange (1811). 
Let us now displace mass $m_{i}$ from its triangular Lagrange point and give it some velocity $\delta n_{i}$. If the system is revolving about the central mass $(M)$ with mean motion $n_{0}$, and the angular separation between two bodies $m_{i}$ and $m_{j}$ is greater than $60^{\circ}$, then $\frac{d\left(\delta n_{i}\right)}{d t}$ will be negative. Since the time derivative of $\delta n_{i}$ is decreasing, the mean motion of $m_{i}$ (denoted by $\left.n_{i}\right)$ will eventually become negative and $\left(\theta_{i}-\theta_{j}\right)$ will decrease until mass $m_{i}$ reaches the Lagrange point. See Figure 17a below.

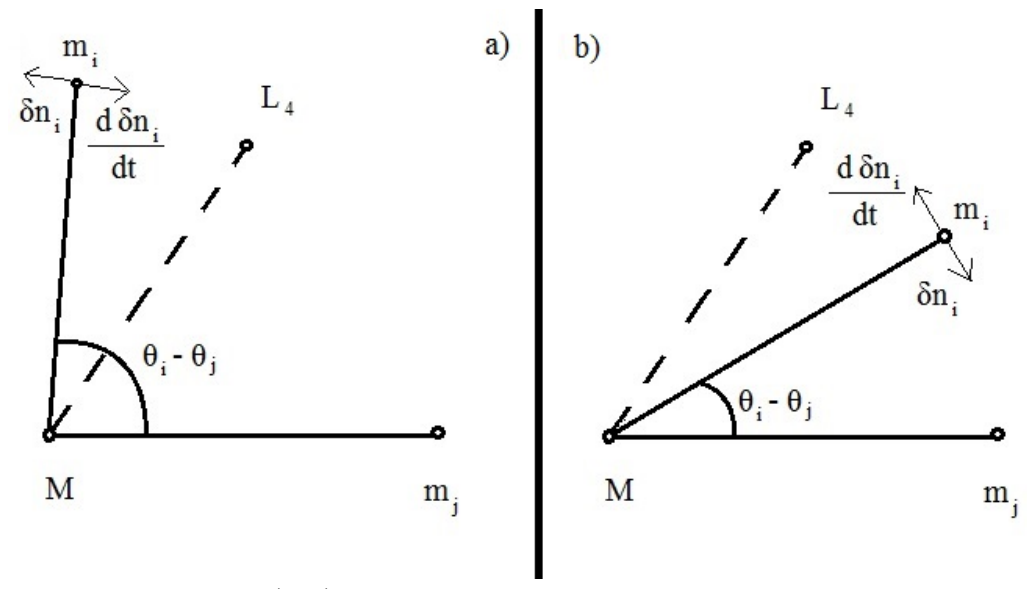

Figure 17. The effect of $\frac{d\left(\delta n_{i}\right)}{d t}$ on $\theta_{i}-\theta_{j}$. Figure 17a shows that $\left(\theta_{i}-\theta_{j}\right)$ is greater than $60^{\circ}$, and $\frac{d\left(\delta n_{i}\right)}{d t}$ will move $m_{i}$ back toward the Lagrange point. In Figure $17 \mathrm{~b}$, the angular separation between $m_{i}$ and $m_{j}$ is less than $60^{\circ}$, and $\frac{d\left(\delta n_{i}\right)}{d t}$ increases $\left(\theta_{i}-\theta_{j}\right)$ until $m_{i}$ reaches the Lagrange point. Note that $\delta n_{i}$ and $\frac{d\left(\delta n_{i}\right)}{d t}$ point in opposite directions.

If the angular separation between $m_{i}$ and $m_{j}$ is greater than $60^{\circ}$ then $\frac{d\left(\delta n_{i}\right)}{d t}$ will be positive (See Figure 17b). Thus, mass $m_{i}$ will be accelerated toward the Lagrange point. We can see that if mass $m_{i}$ is displaced from a triangular Lagrange point or given some velocity at the same Lagrange point, it will oscillate about that point.

However, our system includes four bodies: the Earth, the Moon, and two satellites. Salo and Yoder (1988) were able to show that for three masses of equal magnitude orbiting a much larger central mass, the system will equilibrate when the angular separation between adjacent bodies is $47.4^{\circ}$. Our system is not made up three equal masses orbiting the Earth, however. In Chapter 1, we stated that our 
system will contain the Earth, the Moon, and two near Earth asteroids. Let us put two near Earth asteroids at the $L_{4}$ and $L_{5}$ Lagrange points of the Earth-Moon system.

Recall that the mass of the Moon $\left(m_{j}\right)$ is greater than the mass of the largest near Earth asteroid $\left(m_{i}\right)$ by about seven orders of magnitude. Thus, the Moon's mass will dominate the relative placement of the two satellites, and the system will remain stable with the satellites at the leading and trailing triangular Lagrange points. In fact, since the mass of the Moon is at least some seven orders of magnitude greater than that of any two near Earth asteroids, the two masses need not be the same. The effect of the smaller masses on each other will be dominated by the effect of the Moon on these smaller masses. In summary, any two near Earth asteroids may orbit the Earth at the $L_{4}$ and $L_{5}$ Lagrange points of the Earth-Moon system in a stable orbit. 


\section{CHAPTER 8}

\section{CONCLUDING REMARKS}

It has been known for some time that the three-body problem can be stable as long the mass configuration obeys Gascheau's mass limit. In this thesis, a thorough examination of this stability has been performed, beginning with first principles.

The existence of the Lagrange points has been demonstrated, and the triangular $\left(L_{4}\right.$ \& $L_{5}$ ) Lagrange points have been shown to be stable equilibria as long as the mass limit is obeyed.

In Chapters 4 and 6, we considered several methods of determining this mass limit. One method was to approximate the change in semi-major axis due to the addition of a third mass to a two-body system. The total energy of the two-body configuration was increased proportionately to reflect an overall change in mass for the system of $1 \%$. This is much greater than the corresponding change in mass that the system would experience for any of the near Earth asteroids. If this $1 \%$ threshold is reached, the overall change in the semi-major axis of the system was shown to be much smaller than the overall variation in the semi-major axis of the Earth-Moon System.

Our analysis of the stability of the system led us to Gascheau's work. Unfortunately, the document was not easy to read. This made recreating his original methodology difficult. However, the result has been verified many times over the course of more than a century and a half. Gascheau's result is

$$
\frac{\left(M+m+m^{\prime}\right)^{2}}{M m+M m^{\prime}+m m^{\prime}}>27
$$

During the latter stages of research on this project, the prospect of another possible configuration presented itself. A special case of the Four-Body Problem 
with two identical masses at the triangular Lagrange points produces net forces on the primary and secondary masses directed toward each other and simultaneously the center of mass of the system. Further research showed that the relative angular velocities of the secondary (the Moon) and tertiary (the satellite) masses about the center of mass are affected by the other corotating masses. If two masses have a greater angular separation than $60^{\circ}$, the system will reduce their angular separation; if their angular separation is less than $60^{\circ}$ then the system will increase the angular separation of the two masses. Thus, the system equilibrates when two masses have an angular separation of exactly $60^{\circ}$, just as Lagrange proved. The Moon is several orders of magnitude more massive than any of the near Earth asteroids. Therefore, two near Earth asteroids on opposite sides of the Moon near $L_{4}$ and $L_{5}$ will naturally gravitate toward the triangular Lagrange points where they will remain indefinitely in stable equilibrium. To our knowledge, this specific case has not yet been analyzed.

Examining analogs in our solar system, the stability of Jupiter's Trojan satellites agree with the results presented here (Roy 2005). Perhaps one day one of the near Earth asteroids will have a stable orbit at one of the Earth-Moon System's triangular Lagrange points.

We have shown that as long as the mass limit is obeyed, a mass can remain at one of the triangular Lagrange points indefinitely in a stable orbit. However, this configuration is not only stable for three bodies. We considered a four body system with satellites occupying the both the $L_{4}$ and $L_{5}$ Lagrange points of the Earth-Moon system simultaneously. Any two near Earth asteroids will remain in stable equilibrium at these points. 


\section{REFERENCES}

Archimedes, c. 250 BCE, On Floating Bodies, reissued 1953, T. Heath (Ed.) in The Works of Archimedes with the Method of Archimedes (Dover, New York).

Aristotle, c. 330 BCE, Metaphysics, reissued 1999, H Lawson-Tancred (Ed.) (Penguin, Westminster).

Brown, E. W., and C. A. Shook, 1964, Planetary Theory (Dover, New York,). Copernicus, N., 1543, On the Revolutions of Heavenly Spheres (Johannes Petreius, Nuremburg), reissued (Prometheus, New York).

Cornish, J., 2011, The Lagrange Points (Montana UP, Missoula,).

Danby, J. M. A., 1962, Fundamentals of Celestial Mechanics (Macmillan, New York).

Descartes, R., 1641 Meditations on First Philosophy (René Descartes, London). Einstein, A., 1920, Relativity: The Special and General Theory (Henry Holt, New York).

Galilei, G., 1613, Letters on Sunspots (Giacomo Mascardi, Rome, 1613), reissued 1967, J. Petreium (Ed.) (Culture et Civilisation, Brussels).

Galilei, G., 2000, Starry Messenger (Thomas Baglioni, Venice, 1610) reissued P. Sis (Ed.), (Square Fish, New York).

Gascheau, G., 1843, "Mouvements relatifs d'un système de corps", Faculté des Sciences de Paris.

Kepler, J., 1621, Epitome of Copernican Astronomy (Johannes Kepler), reissued C. Wallis (Ed.) (Prometheus, New York, 1995).

Laërtius, D., c. 230, Lives and Opinions of Eminent Philosophers, reissued 1925, R. Hicks (Ed.) (Harvard UP, Cambridge). 
Lagrange, G., 1811, Mécanique Analytique (Courcier), reissued 2009, (Cambridge UP, Cambridge).

Murray, C. D., and S. F. Dermott, 1999, Solar System Dynamics (Cambridge UP, Cambridge).

Newton, I., 1687, Philosophice Naturalis Principia Mathematica (Edmund Halley, London), reissued 1999, I. Cohen (Ed.) (UC, Oakland).

Plato, c. 380 BCE, Charmides, reissued 1997 J. M. Cooper (Ed.), and D. S. Hutchinson, (Ed.) Plato: Complete Works (Hackett, Indianapolis).

Routh, Edward., 1875, "On Laplace's Three Particles, with a Supplement on the Stability of Steady Motion," Proc. London Math. Soc., 86-97.

Roy, A. E., 2005, Orbital Motion (Institute of Physics, Bristol). Sagan, C., 1980, Cosmos (Random House, New York).

Salo, Heikki, and Charles Yoder, 1988, "The Dynamics of Coorbital Satellite Systems," Astron. Astrophy. 205, 309-327.

Stephenson, 2015, N., Seveneves (William Morrow, New York).

Valsecchi, Giovanni, Ettore Perozzi, Archie Roy, and Bonnie Steves, 1993, "Periodic Orbits Close to that of the Moon," Astron. Astrophys. 271, 308-314.

Nicholson Seth., 1961, "The Trojan Asteroids", Astronomical Society of the Pacific Leaflets, 8, 381, 239.

Yoder, Charles, Guiseppe Colombo, Stephen Synnott, and Kathie Yoder, 1983, "Theory of Motion of Saturn's Co-Orbiting Satellites," Icarus 53, 431-443. 


\section{APPENDIX}

\section{SUMMARY OF GASCHEAU'S ORIGINAL WORK}

In 1843, Gabriel Gascheau published his Mouvements relatifs d'un système de corps in which his Thèse d'astronomie sur deux cas particuliers d'un probleme des trois corps showed that the stability condition for the equilateral triangle

configuration of three masses $\left(M, m\right.$, and $\left.m^{\prime}\right)$ corotating about a common center of mass obeys the following inequality (Gascheau, 1843):

$$
\frac{\left(M+m+m^{\prime}\right)}{M m+M m^{\prime}+m m^{\prime}}>27 .
$$

If one of the masses is taken to be infinitesimal, then the expression reduces to Lagrange's stability condition also called the critical mass ratio. This leads us to the value commonly known as Routh's value.

$$
\begin{gathered}
1-27 \mu(1-\mu) \geq 0, \\
\mu<0.0385 .
\end{gathered}
$$

The derivation is written in slightly archaic French and difficult to follow. The paper itself was not easy to locate, and the quality of the document scan added some dificulty to deciphering the equations.

As shown in Figure 18, several of the characters are not recognizable. Note the second and third lines of equations in particular. Both of these lines begin with the number 4, although it is not altogether apparent or obvious. Immediately following are r's raised to illegibile powers. Most importantly, the difficulty associated with following Gascheau's derivation is not trivial. It is to that end that I have prepared a short summary of this "Thèse d'astronomie." 
92. Equations differenticlles du nouvement trouble: un tant snit pest -

İa suivant une marche analogue à cetle du $\mathrm{n}^{0} \mathbf{1 3}$, un prend

$$
x^{\prime}=\frac{x+\xi-p(y+x)}{2}, \quad y^{\prime}=\frac{y+x+p(x+\xi)}{2},
$$

at l'on a les deux équations

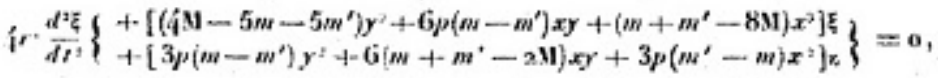

$\left\{r^{4} \frac{d x}{d t^{*}}\left\{\begin{array}{l}+\left[\left(m+m^{\prime}-8 N\right) y^{2}+6 p\left(m^{\prime}-m\right) x y^{2}+\left(\left\{\mathrm{M}-5 m-5 m^{\prime}\right) x^{3}\right] n\right. \\ \left.+\left[3 p\left(m-m^{2}\right) y^{2}+6\left(m+m m^{2}-2 \mathrm{M}\right) x y+3 p\left(m^{\prime}-m\right) x^{2}\right]\right\}\end{array}\right\}=0\right.$.

23. Intégration sles équations du monvement tmublé.

Iin admettant les hypotbèses du $n^{\circ} \mathbf{1 4}$, les deux équations précédentes prenuent la forme de celles du corollaire du $\mathrm{n}^{\circ} \mathbf{2}$. I a constante $\alpha$ est déterminée par l'équation simple ot symétrique

$$
4 m^{2} x^{4}+4 z^{2} x^{2}+3 z\left(\mathrm{Mm}+\mathrm{M} m^{\prime}+m m^{\prime}\right)=0 .
$$

24. Condition de stabilité du mouvement.

It résulte de l'équation précédente quien arrétant les approximations aux premieres dimensions de $\frac{x}{2}$ et $\gamma_{i}$, le monvement scra stable lorsquion aura

$$
\frac{\left(\mathrm{M}+\mathrm{M}+\mathrm{m}^{\prime}\right)^{\prime}}{\mathrm{M} m+\mathrm{M} m^{\prime}+\mathrm{mm}^{\prime}}>2 \% .
$$

Ainsi lä stabilité a lieu si lune des masses est trés-grande par rapport aux deax autres, comme cela arrive pour leSoleil, la Terre et la I,une.

Vu et approuté,

Le 3o Janvier 18 , 3 ,

Le Doves de at Facelte des Sciences,

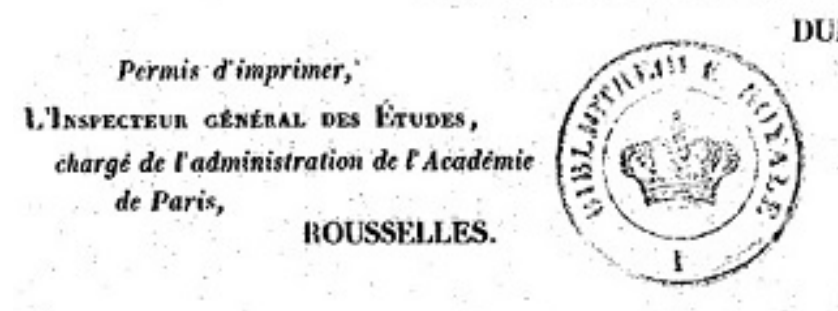

DUMAS.

Figure 18 The final page of Gascheau's work (Gascheau, 1843).

Gascheau begins with Chapter 1, "Linear Differential Equations." In section 1, he begins with an equation of the form

$$
\left(N+N_{1} \tau+\ldots+N_{n} \tau^{n}\right) \frac{d^{2} x}{d \theta^{2}}+\left(P+P_{1} \tau+\ldots+P_{n} \tau^{n}\right) \frac{d x}{d \theta}+\left(Q+Q_{1} \tau+\ldots+Q_{n} \tau^{n}\right) x=0
$$


The $N$ 's, $P$ 's, and $Q$ 's are constants; $\theta$ is the dependent variable, and $\tau=\tan \theta$. The above equation is satisfied with a value of the form

$$
x=(A \cos \theta+B \sin \theta) e^{\alpha \theta} .
$$

$A, B$, and $\alpha$ are undetermined coefficients. Gascheau goes on to specify the following functions in order to shorten the first equation:

$$
\begin{gathered}
H_{i}=N_{i} \alpha^{2}+P_{i} \alpha-N_{i}+Q, \\
K_{i}=2 N_{i} \alpha+P_{i} .
\end{gathered}
$$

The value of $i$ ranges from zero to $n$. $H$ and $K$ satisfy equations of degree $n$.

$$
\begin{gathered}
H-H_{2}+H_{4}-\ldots=-K_{1}+K_{3}-\ldots, \\
K-K_{2}+K_{4}-\ldots=H_{1}-H_{3}+\ldots
\end{gathered}
$$

This led Gascheau to three corollaries:

I) The value of $x$ is exponential.

II) One case of the differential equation is of a higher order.

III) Another case allows a complete integral equation to be obtained.

Section 2 begins with a general case when examining the following two equations:

$$
\begin{aligned}
& \left(N+N_{1} \tau+N_{2} \tau^{2}\right) \frac{d^{2} x}{d \theta^{2}}+\left(P+P_{1} \tau+P_{2} \tau^{2}\right) x+\left(Q+Q_{1} \tau+Q_{2} \tau^{2}\right) y=0, \\
& \left(N_{2}-N_{1} \tau+N \tau^{2}\right) \frac{d^{2} y}{d \theta^{2}}+\left(P_{2}-P_{1} \tau+P \tau^{2}\right) x+\left(Q_{2}-Q_{1} \tau+Q \tau^{2}\right) x=0 .
\end{aligned}
$$

These two equations have the following solutions:

$$
x=(A \cos \theta+B \sin \theta) e^{\alpha \theta},
$$




$$
y=(-B \cos \theta+A \sin \theta) e^{\alpha \theta}
$$

Making the following substitution leads to another corollary.

$$
H_{i}=N_{i} x^{2}-N_{i}+P_{i}, K_{i}+2 N_{i} x-Q_{i}
$$

Gascheau's following corollary states that if the proposed equations were subject to the above method, they would give complete integrals. Unfortunately the first characters in this corollary are illegible, as are the powers of $\tau$. I will substitute $v$ for the missing character and $\varsigma$ for the missing exponent.

$$
\begin{aligned}
& \left(v+\tau^{\varsigma}\right) \frac{d^{2} x}{d \theta^{2}}+\left[P+\left(Q_{2}-Q\right) \tau+P_{2} \tau^{2}\right] x+\left[Q+\left(P-P_{2}\right)+Q_{2} \tau^{2}\right] y=0 \\
& \left(v+\tau^{\varsigma}\right) \frac{d^{2} y}{d \theta^{2}}+\left[P_{2}+\left(Q-Q_{2}\right) \tau+P_{2} \tau^{2}\right] y+\left[Q_{2}+\left(P_{2}-P\right)+Q \tau^{2}\right] x=0
\end{aligned}
$$

Chapter 2, entitled "Known solutions to the three-body Problem," considers both the colinear and equilateral triangle three-body equilibria. Section 3 of this chapter ("Differential Equations of Motion") begins by stating that three point masses $\left(M, m\right.$, and $\left.m^{\prime}\right)$ are mutually attracted, and these attractions are a function $\varphi$ of the distances between them. This system remains in the same plane, with two rectangular axes passing through the center of mass $G$. Gascheau goes on to define several quantities. $X$ and $Y$ are the coordinates of mass $M$ at some time. $X+x$ and $Y+y$ are the position of mass $m$ and $X+x^{\prime}$ and $Y+y^{\prime}$ are the position of mass $m^{\prime}$ at the same time. The distances from $M$ to $m, M$ to $m^{\prime}$, and $m$ to $m^{\prime}$ are given by $r, r^{\prime}$, and $\rho$ respectively. Finally, $\mu$ is the sum of the three masses.

$$
\begin{gathered}
r^{2}=x^{2}+y^{2}=\overline{M m}^{2}, \\
r^{\prime 2}=x^{\prime 2}+y^{\prime 2}={\overline{M m^{\prime}}}^{2},
\end{gathered}
$$




$$
\begin{gathered}
\rho^{2}=\left(x-x^{\prime}\right)^{2}+\left(y-y^{\prime}\right)^{2}={\overline{m m^{\prime}}}^{2}, \\
\mu=M+m+m^{\prime} .
\end{gathered}
$$

Section 4 is entitled "Necessary Conditions for Movement of the Same Nature of Two Bodies." The motion is described by two equations which give the following two solutions:

$$
\begin{aligned}
& \left(x y^{\prime}-y x^{\prime}\right)\left[\frac{\varphi\left(r^{\prime}\right)}{r}-\frac{\varphi(\rho)}{\rho}\right]=0, \\
& \left(x y^{\prime}-y x^{\prime}\right)\left[\frac{\varphi(r)}{r}-\frac{\varphi(\rho)}{\rho}\right]=0 .
\end{aligned}
$$

Section 5 considers the first of these two possible solutions. The attractive forces are proportional to the distances to a power $n$. The constants $k$ and $\lambda$ in these equations are proportionality constants that depend on the relative values of the masses (namely $m$ and $m^{\prime}$ ). The equations of motion are as follows:

$$
\begin{aligned}
& \frac{d^{2} x}{d t^{2}}+\lambda^{2} x r^{n-1}=0, \\
& \frac{d^{2} y}{d t^{2}}+\lambda^{2} y r^{n-1}=0 .
\end{aligned}
$$

Gascheau then sets

$$
\begin{gathered}
x^{\prime}=k x, \\
y^{\prime}=k y, \\
r^{\prime}=k r, \\
\rho=(k-1) r .
\end{gathered}
$$

Gascheau concludes that the three bodies are in a straight line. 
Section 6 details the second solution, which is the solution we are concerned with. He states that the equations of motion and the constants are

$$
\begin{gathered}
\frac{d^{2} x}{d t^{2}}+\frac{\mu x}{r} \varphi(r)=0, \\
\frac{d^{2} y}{d t^{2}} \frac{\mu y}{r} \varphi(r)=0, \\
x^{\prime}=\frac{x-p y}{2}, \\
y^{\prime}=\frac{y+p x}{2}, \\
r=r^{\prime}=\rho, \\
p^{2}=3 .
\end{gathered}
$$

Gascheau concludes that the masses remain at the vertices of an equilateral triangle.

Chapter 3 begins the derivation of the first (colinear) solution. Section 8 describes motion about mass $M$. Expressions for the tangential force, the centripetal force, and the radius of the trajectories are similar to a system with two bodies.

Section 9 (entitled "Movement About the Center of Mass") has four subsections. The first states that each body is attracted to the center of mass. This agrees with Lagrange's conclusion for circular motion: the net force on all objects must be directed towards the center of mass. Subsection 2 states that the three bodies are in a straight line (since we are considering the colinear Lagrange points). The next subsection states that the trajectories are similarly placed and curved. Subsection 4 states that the forces and the velocities of the three bodies are parallel to each other and proportional to their radius vectors. 
Section 10 is entitled, "Initial Conditions of Motion." The first of its three subsections assumes that the masses are in a straight line. The second states that the distances of the masses from the center of mass are dictated by the following ratios:

$$
M G: m G: m^{\prime} G::-\left(m+k m^{\prime}\right): M+(1-k) m^{\prime}: k M+(k-1) m \text {. }
$$

The third subsection indicates that the velocities are parallel and proportional to the above ratios.

Section 11 is entitled "Conditions of Equilibrium of One of the Three Bodies." It states that the other two masses must be equal and equidistant from the primary mass.

Section 12 is called "Application of Newton's Laws." Gascheau states that the equations of motion are

$$
\begin{gathered}
\frac{d^{2} x}{d t^{2}}+\lambda^{2} \frac{x}{r^{3}}=0, \\
\frac{d^{2} y}{d t^{2}}+\lambda^{2} \frac{y}{r^{3}}=0, \\
x^{\prime}=(1+p) x, \\
y^{\prime}=(1+p) y, \\
r^{\prime}=(1+p) r, \\
\rho=p r .
\end{gathered}
$$

The equation which determines the constants $\lambda$ and $p=k-1$ is

$$
M+m+\frac{m^{\prime}}{(1+p)^{2}}-\frac{m^{\prime}}{p^{2}}=\frac{M+m^{\prime}}{(1+p) 3}+\frac{m}{1+p}+\frac{m}{p^{2}(1+p)}=\lambda^{2} .
$$

This gives a single positive value for $p$. This value is less than unity for

$$
M>m^{\prime} .
$$


If $m$ and $m^{\prime}$ are small compared to $M$, Gascheau uses the following approximation:

$$
p=\sqrt[3]{\frac{m+m^{\prime}}{3 M}}
$$

For the Sun-Earth-Moon system the value of $p$ is $\frac{1}{100}$. Gascheau goes on to state that the "Consequence of Laplace" can be drawn from this result. He also says that examining the stability of motion is necessary.

Section 13 details differential equations of perturbation. He changes the values of $x^{\prime}$ and $y^{\prime}$ by perturbing the body $m^{\prime}$ from its position in Section 12 by

$$
\begin{aligned}
& x^{\prime}=(1+p)(x+\xi), \\
& y^{\prime}=(1+p)(y+\eta) .
\end{aligned}
$$

The only condition on the quantities $\xi$ and $\eta$ that Gascheau offers is that they are very small; they are so small in fact that he suggests we neglect all their second order terms. That is, $\xi$ and $\eta$ must be the perturbations on $x^{\prime}$ and $y^{\prime}$. He states that these new variables must obey the following differential equations:

$$
\begin{aligned}
& \frac{d^{2} \xi}{d t^{2}}+\frac{\lambda^{\prime 2}}{r^{3}}\left[\xi-\frac{3 x(\xi x+\eta y)}{r^{2}}\right]=0, \\
& \frac{d^{2} \eta}{d t^{2}}+\frac{\lambda^{\prime 2}}{r^{3}}\left[\eta-\frac{3 x(\xi x+\eta y)}{r^{2}}\right]=0,
\end{aligned}
$$

where

$$
\lambda^{\prime 2}=\frac{M-p m^{\prime}}{(1+p)^{3}}+\frac{m+(1+p) m^{\prime}}{p^{3}}=\lambda^{\prime 2} .
$$

Section 14 "Integration of the Equations of Perturbation," begins with Gascheau's assumption that we neglect the eccentricity of Earth's orbit, yielding: 


$$
\begin{aligned}
& x=r \cos \theta, \\
& y=r \sin \theta .
\end{aligned}
$$

Here $\theta$ is proportional to the time. He goes on to write that these are a special case of Corollary 2 (Section 1 ). If we suppose that $B=1$, the constants $A$ and $\alpha$ are determined by

$$
\frac{\lambda^{2} \alpha^{2}-\lambda^{2}+\lambda^{\prime 2}}{2 \lambda^{2} \alpha}=\frac{2 \lambda^{2} \alpha}{-\lambda^{2} \alpha^{2}+\lambda^{2}+2 \lambda^{\prime 2}}=A
$$

He goes on to say that the values of $\xi$ and $\eta$ are those given in Section 2.

Section 15 shows the values of the constants $A$ and $\alpha$ which are solutions to Equation 8.4. He suggests that they are of the forms

$$
\begin{aligned}
& \alpha,-\alpha, \alpha^{\prime} \sqrt{-1},-\alpha^{\prime} \sqrt{-1} \\
& A,-A, A^{\prime} \sqrt{-1},-A^{\prime} \sqrt{-1}
\end{aligned}
$$

$A, A^{\prime}, \alpha$, and $\alpha^{\prime}$, are all real and positive. The following ratios are not equal:

$$
\frac{A}{\alpha} \neq \frac{A^{\prime}}{\alpha^{\prime}}
$$

Section 16 is entitled "Detemination of the Constants of Integration." He states that these are based upon the initial conditions of motion, noting that they cannot be infinite.

The title of Section 17 is "State of Motion of Three Bodies." He writes that whatever the initial conditions may be, the motion is determined by the equations of motion in Section 12. Furthermore, the motion is unstable.

Chapter 4 details the development of the second solution, the vertices of the equilateral triangle or the $L_{4}$ and $L_{5}$ Lagrange points. It begins with Section 18, "Motion About Mass M." 
Section 19 is "Motion About the Center of Mass." The first of three subsections states that the motion of the bodies is the same as a body attracted to the center of mass. The second states that the triangle determined by the three point masses is equilateral. The third and final subsection states that the trajectories are similar.

Section 20 which is entitled "Initial Conditions of Movement" also has three subsections. The first states that the three bodies occupy the three vertices of an equilateral triangle. The second states that the velocities have equal inclinations to their radius vectors from the center of mass; this is equivalent to restricting motion to a plane. The third and final subsection states that these velocities are proportional to the magnitude of the corresponding radius vectors.

Section 21 is "Application of Newton's Laws". He states that the equations of motion are

$$
\begin{gathered}
\frac{d^{2} x}{d t^{2}}+\frac{\mu x}{r^{3}}=0, \\
\frac{d^{2} y}{d t^{2}}+\frac{\mu y}{r^{3}}=0, \\
x^{\prime}=\frac{x-p y}{2}, \\
y^{\prime}=\frac{y+p x}{2}, \\
r=r^{\prime}=\rho .
\end{gathered}
$$

Section 22 is entitled, "Differential Equations of Motion for a Small Perturbation." Following a procedure analagous to that of Section 13, the expressions for the position of mass $m^{\prime}$ become

$$
\begin{aligned}
& x^{\prime}=\frac{x+\xi-p(y+\eta)}{2}, \\
& y^{\prime}=\frac{y+\eta+p(x+\xi)}{2} .
\end{aligned}
$$


This leads us to the two aforementioned difficult to read equations in Figure 18. Section 23, "Integration of the Perturbed Equations of Motion," begins by assuming the "hypotheses" in Section 14 (assuming a circular orbit). He then states that the two illegible equations from Section 22 take the form of the corollary from Section 2. Gascheau states that the value of $\alpha$ can be determined by the simple symmetric equation:

$$
4 \mu^{2} x^{4}+4 \mu^{2} x^{2}+27\left(M m+M m^{\prime}+m m^{\prime}\right)=0 .
$$

In the final section, Section 24 (entitled "Stability Condition for Motion"), Gascheau states that when $\xi$ and $\eta$ are taken only to first order the motion of the bodies will be stable as long as

$$
\frac{\left(M+m+m^{\prime}\right)^{2}}{M m+M m^{\prime}+m m^{\prime}}>27 .
$$

Gascheau's closing remark is that the system is stable if one of the masses is very large compared to the other two, such as that of the Sun-Earth-Moon system. 